\title{
Multiscale Modeling of the Indentation of Nickel-Aluminum Nano-layers
}

\author{
by \\ Ishraq Shabib \\ B.Sc. in Mechanical Engineering, \\ Bangladesh University of Engineering and Technology, \\ Dhaka, Bangladesh. \\ A thesis submitted to the \\ Faculty of Graduate Studies and Research \\ in partial fulfillment of the requirements for the Degree of \\ Master of Applied Science \\ Ottawa-Carleton Institute for Mechanical and Aerospace Engineering
Department of Mechanical and Aerospace Engineering
Faculty of Engineering
Carleton University
Ottawa, Canada
}

August 2005

(c) Ishraq Shabib, 2005 


$\begin{array}{ll}\begin{array}{l}\text { Library and } \\ \text { Archives Canada }\end{array} & \begin{array}{l}\text { Bibliothèque et } \\ \text { Archives Canada }\end{array} \\ \begin{array}{l}\text { Published Heritage } \\ \text { Branch }\end{array} & \begin{array}{l}\text { Direction du } \\ \text { Patrimoine de l'édition }\end{array} \\ \begin{array}{l}\text { 395 Wellington Street } \\ \text { Ottawa ON K1A ON4 }\end{array} & \begin{array}{l}\text { 395, rue Wellington } \\ \text { Ottawa ON K1A ON4 } \\ \text { Canada }\end{array}\end{array}$

Your file Votre référence

ISBN: 0-494-10092-3

Ourfile Notre référence

ISBN: 0-494-10092-3

NOTICE:

The author has granted a nonexclusive license allowing Library and Archives Canada to reproduce, publish, archive, preserve, conserve, communicate to the public by telecommunication or on the Internet, loan, distribute and sell theses worldwide, for commercial or noncommercial purposes, in microform, paper, electronic and/or any other formats.

The author retains copyright ownership and moral rights in this thesis. Neither the thesis nor substantial extracts from it may be printed or otherwise reproduced without the author's permission.
AVIS:

L'auteur a accordé une licence non exclusive permettant à la Bibliothèque et Archives Canada de reproduire, publier, archiver, sauvegarder, conserver, transmettre au public par télécommunication ou par l'Internet, prêter, distribuer et vendre des thèses partout dans le monde, à des fins commerciales ou autres, sur support microforme, papier, électronique et/ou autres formats.

L'auteur conserve la propriété du droit d'auteur et des droits moraux qui protège cette thèse. $\mathrm{Ni}$ la thèse ni des extraits substantiels de celle-ci ne doivent être imprimés ou autrement reproduits sans son autorisation.
In compliance with the Canadian

Privacy Act some supporting forms may have been removed from this thesis.

While these forms may be included in the document page count, their removal does not represent any loss of content from the thesis.
Conformément à la loi canadienne sur la protection de la vie privée, quelques formulaires secondaires ont été enlevés de cette thèse.

Bien que ces formulaires aient inclus dans la pagination, il n'y aura aucun contenu manquant.

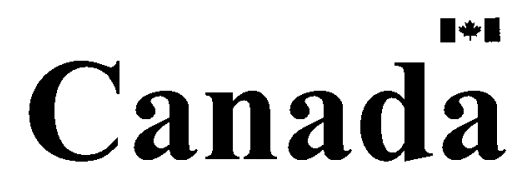


The undersigned hereby recommend to

the Faculty of Graduate Studies and Research

acceptance of this thesis

"Multiscale Modeling of the Indentation of Nickel-Aluminum Nano-layers"

submitted by

Ishraq Shabib, B.Sc.

In partial fulfillment of the requirements for the degree of

Master of Applied Science

Thesis Co-supervisor,

Professor Ronald E. Miller

Thesis Co-supervisor,

Dr. Linruo Zhao

Chair, Department of Mechanical and Aerospace Engineering

Professor J.C. Beddoes

Carleton University

August, 2005

ii 


\begin{abstract}
The present research work presents the multiscale modeling of nano-indentation into nickel-aluminum nano-layers using the coupled atomistics and discrete dislocation (CADD) model. In this work, the model has been extended to study the layered structures to reveal how the microscopic mechanisms lead to the observed indentation responses. Simulation results showed that the misfit dislocations at the interface played an important role to initiate the plastic deformation in the layered structures. The movements of misfit dislocations and their dissociation events from the interface were found to be affected largely by the stress field induced by the indenter. Load-displacement and hardness-displacement plots for different $\mathrm{Ni}-\mathrm{Al}$ models were evaluated and their responses were compared with the behaviors of the pure metals. The hardness trends for all simulation models displayed their dependence upon simultaneous contributions from the indentation size effect (ISE) and the hardening effect. It was also observed that the atomistic consideration near the interface captured the dislocation/interface interactions more accurately compared to the continuum model.
\end{abstract}




\section{Acknowledgements}

First of all I want to express my sincere gratitude to the Almighty Allah to give me the strength, knowledge and patience to complete this task, which I believe the most significant accomplishment in my life. Since last two years I have been accompanied and supported by many people and it is now a pleasant opportunity to express my gratitude to all of them.

I am deeply indebted to my supervisor Professor Ronald E. Miller whose wisdom, guidance, and encouragement helped me to successfully finish this research work. He taught me sincerely every important aspect of materials science and also showed me the way to do the computational works efficiently. He was always there to listen my problems and give me the advice. I am very glad to work with him.

I would like to share this moment of happiness with my parents who rendered gargantuan love and encouragement from home during the tenure of my research. I am obliged for the enormous sacrifice they have been made since my childhood. Especially, I am gratified to my sister, 'Lora', for the support and encouragement she provided both to me and to our parents during my stay in Canada. I would like to give my special thanks to my wife 'Lagna' for the inspiration, love and moral support she provided throughout my research work. Her patience was tested to the utmost by a long period of separation. Without their loving support and understanding I would never have completed my work.

I wish I would never forget the company I had with my co-worker 'Gladys'. I greatly appreciate her thoughts, ideas and valuable hints in solving numerous problems. I am thankful to the fellow research scholars from my Department, 'Behrouz Shiari' and 'Denis Saraev', for their valuable suggestions and guidance to carry out this research. I also gratefully acknowledge the cooperation that I received from other faculty members and administrative staffs of my department. I want to give my spe- 
cial thanks to Dr. Linruo Zhao and Dr. Kuiying Chen from Institute of Aerospace Research (IAR) of NRC, for their cooperation and valuable comments regarding my thesis.

I would also like to express my gratitude to the Department of Mechanical and Aerospace Engineering and the Faculty of Graduate Studies and Research of Carleton University and National Research Council (NRC) of Canada for giving me the financial support throughout my masters program. 
To my parents 


\section{Contents}

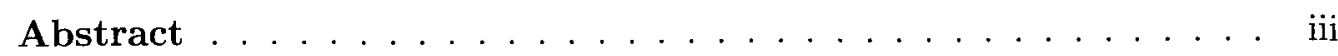

Acknowledgements ................. iv

Table of Contents . . . . . . . . . . . . . vii

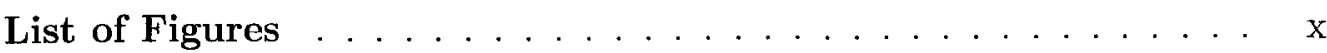

List of Tables . . . . . . . . . . . . . . . . . xiii

Nomenclature ....................... . . . . . . . . . . . .

1 Introduction 1

1.1 Multilayer structures and their properties . . . . . . . . . . . . 1

1.2 Nano-indentation . . . . . . . . . . . . . . . . 3

1.3 Atomistic and multiscale modeling . . . . . . . . . . . 3

1.4 Motivation of this research . . . . . . . . . . . . . . . 4 4

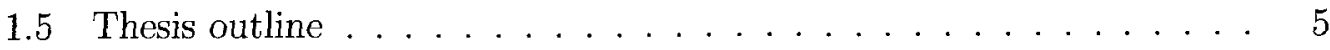

2 Background Material and Literature Review $\quad 7$

2.1 Preparation of thin films . . . . . . . . . . . . . . 8

2.1 .1 Physical Vapor Deposition . . . . . . . . . . . . . . 8

2.1 .2 Chemical Vapor Deposition . . . . . . . . . . . 10

2.2 Film growth . . . . . . . . . . . . . . . . 11

2.3 Applications of thin films . . . . . . . . . . . . . . 11

2.3 .1 Electrical and Magnetic . . . . . . . . . . . . 11

2.3 .2 Metallurgical and protective coatings . . . . . . . . . . 12

vii 
2.3 .3 Optical . . . . . . . . . . . . . . 12

2.4 Nano-indentation . . . . . . . . . . . . . . . . 12

2.4.1 Interpretation of load-displacement curve . . . . . . . 13

2.4.2 Stiffness, Contact area, Modulus and Hardness measurement . 14

2.4.3 Factors affecting nano-indentation test data . . . . . . . 18

2.5 Hardening theories at the nanometer scale . . . . . . . . . 22

2.5 .1 Orowan Model: . . . . . . . . . . . . . . . 22

2.5.2 Hall-Petch Model: . . . . . . . . . . . . . . . . . . . . . 23

2.5.3 Dislocation-Interface Interaction . . . . . . . . . . . 26

2.6 Computational approach $\ldots \ldots \ldots \ldots$

2.6 .1 Atomistic modeling scheme $\ldots \ldots \ldots . \ldots 32$

2.6.2 Multiscale modeling scheme . . . . . . . . . . . . . . 35

3 Coupled Atomistic and Discrete Dislocation (CADD) Method 42

3.1 Continuum Solution Procedure . . . . . . . . . . . . . . . 44

3.2 Atomistic Solution Procedure . . . . . . . . . . . . . . . 47

3.3 Equilibrium configuration . . . . . . . . . . . . . . . 48

3.4 Dislocation Detection and Passing . . . . . . . . . . . . . . . 49

3.4.1 Dislocation Detection . . . . . . . . . . . . . . . 49

3.4 .2 Dislocation Passing . . . . . . . . . . . . . . . . . 50

3.5 Modification of the CADD model . . . . . . . . . . . . 51

3.5.1 Selection of the potential . . . . . . . . . . . 53

3.5.2 Lattice constant calculation for $\mathrm{Al}$ and $\mathrm{Ni} \ldots \ldots . . . . .55$

3.5.3 Elastic modulus Calculation . . . . . . . . . . . . 57

3.5.4 Model description for bilayer simulation . . . . . . . . 59

3.5.5 Model description for multilayer simulation . . . . . . . . . 63

viii 
4 Results and analysis $\quad 68$

4.1 Bilayer simulation results . . . . . . . . . . . . . 68

4.1.1 Aluminum film on top of nickel substrate . . . . . . . . . 69

4.1.2 Nickel film on top of aluminum substrate . . . . . . . . . 73

4.1.3 Load-Displacement curves for Al-Ni bilayer systems . . . . . . 76

4.1.4 Hardness measurement for bilayer systems . . . . . . . . . . 78

4.1.5 Atomic level stress calculation . . . . . . . . . . . . . . 82

4.2 Multilayer simulation results . . . . . . . . . . . . . 87

$4.2 .1 \quad$ Ni-Al-Ni system . . . . . . . . . . . . . . . . . . . 88

4.2.2 Ni-Al-Ni system (without an atomistic region at the second interface $\ldots \ldots \ldots \ldots \ldots \ldots$

4.2.3 Load-Displacement curves for $\mathrm{Ni}-\mathrm{Al}$ multilayer systems . . . . 91

4.2.4 Hardness measurement for multilayer systems . . . . . . . . . 93

5 Conclusions and Future Recommendations 96

5.1 Summary of conclusions . . . . . . . . . . . . 96

5.2 Recommendations for future work . . . . . . . . . . . . . . . 99 


\section{List of Figures}

1.1 Multilayer coating structure on Ti-6Al-4V substrate. . . . . . . . . 5

2.1 Load vs. Displacement curve . . . . . . . . . . . . . . . . . . . 14

2.2 A cross section through an indentation showing various quantities used in the analysis . . . . . . . . . . . . . 17

2.3 Schematic of the initial penetration depth on load vs. displacement data ............................. 19

2.4 Geometrically necessary dislocations created by a rigid conical indenter 21

2.5 Effect of sinking-in and pile-up on the actual contact area . . . . . . 22

2.6 Single-dislocation based Orowan model . . . . . . . . . . . . . 23

2.7 Pile-up of dislocations against a grain boundary under an applied shear stress . . . . . . . . . . . . . . . . . . . 24

2.8 Hall-Petch plot for several Cu-based multilayers . . . . . . . . . 26

2.9 Experimental observations and model predictions for tensile yield stress vs. superlattice period for $\mathrm{Cu} / \mathrm{Ni}$ superlattices . . . . . . . . 29

2.10 Experimental observations and model predictions for hardness vs. superlattice period for $\mathrm{TiN} / \mathrm{NbN}$ superlattices . . . . . . . . . . 30

2.11 Schematic illustration of the solution procedure of the discrete dislocation method . . . . . . . . . . . . . . . 37

2.12 The atomistic/continuum region for the QC method . . . . . . . 38

2.13 The atomistic/continuum region for the FEAt method . . . . . . . 40

2.14 The atomistic/continuum region for the CLS method . . . . . . . . 41 
3.1 Schematic illustration of the solution procedure for the CADD method 45

3.2 Position of the detection band to detect dislocations . . . . . . 50

3.3 Dislocation detection and passing across the atomistic/continuum interface in the CADD method $\ldots \ldots \ldots \ldots . \ldots . \ldots 5$

3.4 CADD flow chart . . . . . . . . . . . . . . . 52

3.5 The functions comprising the $\mathrm{Ni}, \mathrm{Al}$ and $\mathrm{Ni}_{3} \mathrm{Al}$ potentials . . . . . . 54

3.6 Bravais lattice vectors of Aluminum and Nickel _ . . . . . . . . . 58

3.7 Bilayer simulation model . . . . . . . . . . . . . . 61

3.8 Atomistic region and the position of the layer interface between $\mathrm{Ni}$

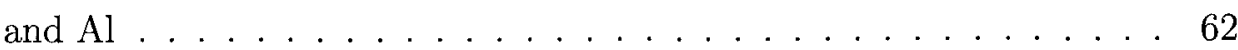

3.9 Positions of the real atoms, interface atoms, pad atoms and the continuum nodes in bilayer simulation model . . . . . . . . . . . . . . 63

3.10 Actual position of the interface in the continuum region . . . . . . 64

3.11 Multilayer simulation model . . . . . . . . . . . . . . . . 65

3.12 Positions of real atoms, interface atoms, pad atoms and continuum nodes around the second atomistic region . . . . . . . . . . . 66

3.13 Position of the detection band inside the second atomistic region . . . 67

4.1 Positions of the misfit dislocations along the interface of the $\mathrm{Al} / \mathrm{Ni}$ model ......................... . . 70

4.2 Movements of the misfits and their dissociation from the interface . . 71

4.3 Dissociation of the misfit dislocations at different locations in the layer

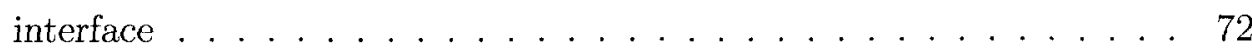

4.4 Dissociation of dislocation inside the layer f . . . . . . . . . . . 72

4.5 Accumulation of the dislocations in the aluminum layer . . . . . . 73

4.6 Position of the misfits along $\mathrm{Ni} / \mathrm{Al}$ interface $\ldots \ldots . . . . .74$

4.7 Movement and dissociation of the misfit dislocations at the $\mathrm{Ni} / \mathrm{Al}$ interface ......................... 75 
4.8 Passing of dislocation across the $\mathrm{Ni} / \mathrm{Al}$ interface $\ldots \ldots . . . .76$

4.9 Load-Displacement curves for different Al-Ni bilayer system . . . . 77

4.10 Hardness variation with depth of penetration for different bilayer models 79

4.11 Hardness trends after first dislocation nucleation for different bilayer models . . . . . . . . . . . . . . . . . . 81

4.12 Residual stress distribution along the $\mathrm{Ni} / \mathrm{Al}$ interface . . . . . . 83

4.13 The effect of indenter on the atoms in the atomistic region at different indentation depth . . . . . . . . . . . . . . 84

4.14 Shear stress variation of the atoms located at the interface . . . . 85

4.15 Shear stress variation of the atoms located at $10.0 \AA$ above and below

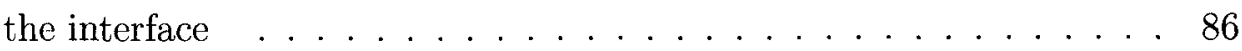

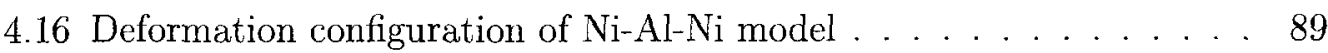

4.17 Deformation configuration of $\mathrm{Ni}-\mathrm{Al}-\mathrm{Ni}$ (without 2 nd atomistic region) model ........................... . 90

4.18 Load-Displacement curves for different Al-Ni multilayer system . . . . 92

4.19 Hardness variation with depth of penetration for different multilayer models . . . . . . . . . . . . . . . . . . . . 94

4.20 Hardness trends after first dislocation nucleation for different multilayer models . . . . . . . . . . . . . . . . . . . . . . 95 


\section{List of Tables}

3.1 Experimental and calculated properties of aluminum . . . . . 55

3.2 Experimental and calculated properties of nickel . . . . . . . 5 55

3.3 Calculated in-plane elastic constants for aluminum . . . . . . . . . . 59

3.4 Calculated in-plane elastic constants for nickel . . . . . . . . . . 59

4.1 Summary of the results obtained from all bilayer models . . . . . . 82

4.2 Summary of the results obtained from all multilayer models . . . . . 94 


\section{Nomenclature}

\begin{tabular}{|c|c|}
\hline CADD & Coupled atomistic and discrete dislocation method \\
\hline $\mathrm{QC}$ & Quasicontinuum method \\
\hline CLS & Concurrent length scale method \\
\hline FEAt & Finite element and atomistic method \\
\hline EAM & Embedded atom method \\
\hline ISE & Indentation size effect \\
\hline $\mathrm{Al} / \mathrm{Ni}$ model & Aluminum film on top of a nickel substrate \\
\hline $\mathrm{Ni} / \mathrm{Al}$ model & Nickel film on top of an aluminum substrate \\
\hline Al-Ni-Al model & Aluminum and nickel film on top of an aluminum substrate \\
\hline $\mathrm{Ni}-\mathrm{Al}-\mathrm{Ni}$ model & Nickel and aluminum film on top of a nickel substrate \\
\hline $\mathrm{X}$ & Width of the model $(\AA)$ \\
\hline $\mathrm{Y}$ & Thickness of the model $(\AA)$ \\
\hline $\mathrm{P}$ & Indenter load $(e V / \AA)$ \\
\hline$\delta$ & Displacement of the indenter $(\AA)$ \\
\hline A & Area of contact $\left(\AA^{2}\right)$ \\
\hline $\mathrm{H}$ & Hardness $\left(e V / \AA^{3}\right)$ \\
\hline$\Phi$ & Pair potential \\
\hline $\mathrm{F}$ & Embedding energy \\
\hline$\rho$ & Electron density \\
\hline$r_{c l}$ & Cutoff radius \\
\hline
\end{tabular}


$\mathrm{R}$

$E^{*}$

E

$\nu$

G

$\lambda, \mu$

b

$\Lambda$

$\mathrm{m}$

$\lambda_{c}$

$\sigma_{i j}$

$\epsilon$

$\mathrm{u}$

$a_{0}$

$C_{11}, C_{12}, C_{66}$

$\Omega_{i}$
Interatomic distance between atoms

Relative radius of curvature

Reduced Modulus

Young's Modulus

Poisson's ratio

Shear Modulus

Voigt-average elastic constants

Burger's vector

Bilayer period

Slip plane normal

Coherency wavelength limit

Atomic level stress tensor

Strain

Displacement

Lattice constant

Elastic constants

Atomic volume at site $i$ 


\section{Chapter 1}

\section{Introduction}

\subsection{Multilayer structures and their properties}

Measuring and understanding the mechanical properties of thin film structures, consisting of alternating nano-scale multilayers, have been a recent area of active investigation due to their enhanced hardness, tensile strength, corrosion resistance and wear resistance properties compared to monolithic coatings of the constituent materials $[1],[2]$. Some of the nanolayered metals, ceramics and alloys even exhibit super-elasticity that ensures large elongation of solids without failure at relatively low temperature and high strain rates [3]. Early multilayer coatings consisted of a layer of nitride or carbide and an oxide, such as $\mathrm{TiN}$ or $\mathrm{TiC}$ and $\mathrm{Al}_{2} \mathrm{O}_{3}$, and were used to improve the life of cutting tools. The nitride/carbide layer ensures strength and the oxide layer provides corrosion resistance to make the structure very useful in cutting applications. Though the thickness of the individual layers of early multilayer coating was of several microns thick, a new class of multilayer structure has emerged that consists of very thin $(2-10 \mathrm{~nm})$ layers and exhibits extremely high hardness [4]. Experimental results also showed that metallic multilayered structures made of two kinds of metals, even those made from soft metals, exhibit significant increase in yield and fracture strengths with decreasing bilayer thickness $[1],[5]$. This enhancement is not easily attainable by traditional microstructures. However, below a critical bilayer period, the hardness values may remain constant or even decrease 
with bilayer thickness [1]. The secret of such behavior actually lies in the plastic deformation of these materials. Over the years, understanding of plastic deformation of materials at nanometer length scale is a challenge in materials science. The plastic behavior of materials at such a small length scale is mainly controlled by the nucleation and motion of lattice dislocations [6]. Although, dislocations are simple line defects of the materials, the ways those defects nucleate and interact with each other and with grain boundaries, are very complex in nature [7]. In coarse grained materials the motion of dislocations within individual grains is hindered by the grain boundaries, which results in dislocation pile-ups against them. Further reduction in grain size introduces more obstacles to the dislocation motion, thereby making metal harder to deform. This strengthening mechanism is expressed by the Hall-Petch relation. However, at very small grain sizes the Hall-Petch relation breaks down and even becomes a reverse one [8]. Moreover, there are some other factors, i.e. modulus mismatches, lattice parameter mismatches, gamma surface mismatches and slip-plane mismatches between adjacent layers, that have also been attributed for this enhancement [9]. A detail discussion about hardness enhancement at nanometer length scale will be presented in Chapter 2. Though very high hardness or strength has already been observed in many nanomaterials, in most of the cases they have low ductility due to the physical nature of plastic deformation. But several investigations have also been carried out for materials having both of these two opposing characteristics. Wang et al. [10] created a "bimodal" structure of micrometer-sized grains embedded in a matrix of nanocrystalline grains that showed extraordinarily high ductility, but also retained its high strength. In such structures, nanocrystalline grains provide strength and the embedded larger grains stabilize the tensile deformation of the material [10]. A few authors have proposed a so-called rotational plasticity [11] that also led to high strength and ductility in nanostructured materials. Such occurrence became possible through the formation of a nanometer sized 
grain structure in which sliding can occur at grain boundaries. It is thus necessary to explore all these underlying mechanisms that contribute to the enhanced mechanical properties of materials that are highly desirable for engineering applications and can be exploited to make "next-generation" materials .

\subsection{Nano-indentation}

During the last several years, nano-indentation testing has become an integral part of modern materials science to characterize the mechanical behavior of materials of very small volume. Although the test involves simply penetrating a specimen of interest with an indenter, the information that can be achieved from this test has much importance. In practice, the goal of such testing is to measure different mechanical properties, i.e. elastic modulus, hardness and contact stiffness, of the specimen under consideration from indenter load and penetration depth. Several researchers proposed several ways to interpret the load-displacement curves and to extract data from it for different materials, and the results showed good estimation of such materials' properties. Present research work is thus focused on simulating such nano-indentation testing into layered materials to elucidate the underlying mechanisms that contribute to the overall response of the system.

\subsection{Atomistic and multiscale modeling}

As we have discussed in our previous sections there are important phenomena occurring at many different length scales that contribute to mechanical properties. These length scales range from angstrom to micrometer. Since experimental measurements and conventional theoretical modeling based on a continuum approach cannot offer a microscopic view of the physics at such small length scales, atomistic simulations are becoming increasingly prominent in materials science to elucidate the effects of defects, surfaces and other microstructural features. Predictions re- 
sulting from this atomic-level understanding are a vital source of information for directing experimental research. Fully atomistic simulations are essential to analyze atomic scale phenomena, but may involve millions of atoms to simulate the whole structure. This may reduce the performance of computers for their limitations in memory requirements and simulation time. To overcome this problem a multi-scale material modeling approach has emerged, whereby atomistic modeling is used in conjunction with a continuum approach to link both time and length scales. In the multi-scale modeling approach, atomistic calculation is used in regions where atomic scale defect nucleation and interaction effects are important, whereas a continuum scheme is applied to the rest of the system by dividing it into linear elastic finite elements to reduce overall computation time $[12],[13],[14]$.

\subsection{Motivation of this research}

Nanolayer coatings find their applications in gas turbine engines in the aerospace industry and also in the high-speed machining tools to protect erosion and wear. Figure 1.1 shows a multilayer coating on $\mathrm{Ti}$ substrate, which consists of $\mathrm{Ti}, \mathrm{Cr}$ and TiN/CrN superlattice. Multilayer coating makes the structure hard, tough and environmental resistant. Hardness of such structure can be as high as 20-40 GPa. TiN is hard compared to $\mathrm{CrN}$, but the TiN/CrN superlattice coating exhibits much higher hardness in comparison with monolayered $\mathrm{TiN}$ and $\mathrm{CrN}$ coating. In our present research work two different types of materials, $\mathrm{Ni}$ and $\mathrm{Al}$, have been selected to create different film-substrate systems to simulate this physical phenomenon. One of our main objectives was to understand the different atomic scale phenomena, i.e. dislocation nucleation and dislocation-grain boundary interactions that contribute to the macroscopic mechanical behavior of these film-substrate systems. A new multiscale modeling approach, Coupled Atomistic Discrete Dislocation (CADD) method, has been adopted to simulate the behavior of these systems during nano-indertation. 


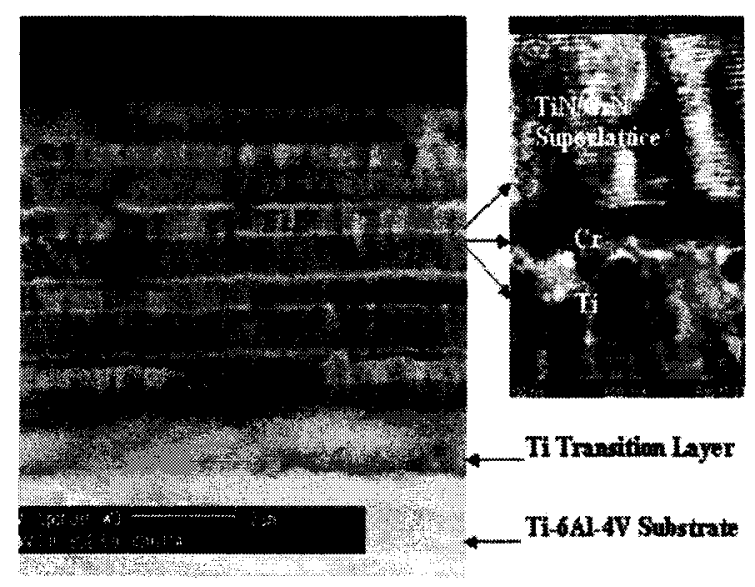

Figure 1.1: Multilayer coating on Ti-6Al-4V substrate. The multilayer coating consists of Ti, Cr and TiN/CrN superlattice (Picture obtained from NRC, ACE2003AMTC, Sept 11, 2003).

\subsection{Thesis outline}

This thesis is divided into five chapters. This chapter provides a brief introduction about multilayered structures at the nanometer length scale, their properties and applications, different modeling schemes and motivation of this work. Chapter 2 is the literature review part of this thesis. It is mainly divided into two parts: a brief discussion about different physical problems and the methods of study of those physical problems. In the first part we will be discussing about thin films, their characteristics, preparation and applications. Moreover, nano-indentation in thin metal films and strengthening theories at the nanometer length scale will also be discussed in this part. In the second part, a brief review of atomistic modeling and different multiscale modeling schemes will be presented. Results from atomistic simulation of nano-indentation on bilayered and multilayered thin films will be discussed and some results from experimental work on metal thin films will also be presented. Chapter 3 will mainly be focused on the coupled atomistic and discrete dislocation (CADD) method. This is a multiscale modeling scheme that has been used to simulate the problems in this thesis. The theory and solution procedure of the CADD method 
has already been published in [15],[16]. A brief review of the theory and solution procedure will be discussed first and then the details of our modifications to the existing CADD code will be presented. Simulations were pcrformed on different bilayered and multilayered film-substrate systems. Nano-indentation simulation results and analysis of those results will be presented in Chapter 4. Chapter 5 presents concluding remarks based on the results obtained from the present work and also gives recommendations for future work. 


\section{Chapter 2}

\section{Background Material and Literature Review}

Understanding of the fundamental nature of materials has dramatically increased throughout much of the twentieth century that led to the development of materials science and engineering. During this period a wide variety of metallic, polymeric, nuclear, ceramic and electronic materials have emerged, and a number of reliable methods to process these materials in bulk and thin-film forms have been developed. Thin film processing is one of the oldest arts and one of the newest sciences that have been subjected to countless investigations since the first films were fabricated more than one hundred years ago. They were first employed as coatings of precious metals and later found wide applications for corrosion protection. But in recent years thin films play a crucial role in the high tech industries. Major exploitation of thin films has been in microelectronics, whereas there are numerous and growing applications in communications, optical, electronics, magnetic and tribological fields [17]. Thin film structures in form of nano-scale multilayers exhibit high temperature stability, enhanced hardness, yield strength, corrosion and chemical resistance properties that make them very useful as a coating of the cutting tools, turbine and compressor blades to improve their life $[4],[2],[18],[8],[19],[5],[20]$. The term "thin film" refers to a very thin layer of gaseous, liquid or solid material, the thickness of which is usually regarded as being between $2-10000 \AA$. Because of extreme thinness, thin 
films have very little strength and cannot be made self-supporting. Thus, they must be fabricated by deposition on to a suitable substrate. The main role of the substrate is to provide mechanical support of the thin film [21]. The primary interest in thin films is due to two effects. Firstly, a material in thin-film form sometimes has important properties that differ from those of the material in bulk. As the film becomes thinner the surface-to-volume ratios becomes larger and consequently the surface usually influences the film's properties greatly. The other cause is the possibility of the miniaturization of electronic equipments, where thin films find one of their most useful applications [22], [21].

\subsection{Preparation of thin films}

There are number of methods available to fabricate thin films. The method to be used will depend on the material to be deposited and the substrate itself. Among numerous techniques, physical vapor deposition (PVD) and chemical vapor deposition (CVD) are probably the most widely used techniques for the preparation of thin films [23]. In this section a brief review of these methods will be presented.

\subsubsection{Physical Vapor Deposition}

Physical vapor deposition (PVD), the term that includes both evaporation and sputtering $[17]$.

\section{Evaporation}

This technique is by far the most used for the deposition of thin films. It is very simple in essence. A vacuum or reduced pressure environment is created within a chamber by a vacuum pump. The exact pressure used will depend upon the particular type of film being produced. Within the chamber the material of which the thin film is to be made, and the substrate on which the film is to be deposited are placed. The thin film material acts as a source of vapor. The vapor source 
is usually placed in either a tungsten basket or an alundum crucible. Tungsten wires, refractory metal sheets, electron beams can act as a source of heat. Thin film material is heated until the metal vapor is evolved. Due to low pressure within the chamber the molecules radiate out from the source and deposit on the substrate [22]. The evaporation rate, film uniformity and film purity depend upon the atomic weight and the vapor pressure of the element, temperature and evaporation coefficient, vacuum pressure in the system and the design of the source. These factors not only affect the vaporization rate but also through chemical reactions during evaporation and condensation can profoundly modify the structural, electrical and optical properties of the film [23]. Moreover, the impurities that are initially present in the source and the heater and those originate from the residual gases present in the vacuum system have a profound effect in degrading many of the properties of evaporated films [17].

\section{Sputtering}

When atoms having high energies strike a surface, it forces the material from the surface to be ejected. This process is termed as "sputtering". The material to be deposited on the substrate in the form of thin films is known as "target plate". It is often termed as "cathode", since it is connected to the negative terminal of the power supply. The substrate that faces the cathode may be grounded, electrically floating, biased positively or negatively, heated, cooled or some combination of these. The vacuum pressure is maintained in the system and a neutral gas, typically argon, is allowed to flow through the system while the sputtering takes place. This neutral gas serves as the medium in which a discharge is initiated and sustained. Microscopically, positive ions in the discharge strike the cathode plate and eject atoms from it through momentum transfer. The yield depends upon the properties of the target material, the energy and type of positive ions, and also on the geometric configuration. These released atoms are then become available to be deposited on 
a suitably placed substrate. A particular advantage of the sputtering technique is that a uniform thickness of film can be obtained over a large area. It also allows a much better control of composition when producing alloy films. This technique also allows flexibility in alternation of film composition if required [22],[17]. The sputtering process can be divided into four categories: (1) dc (2) RF (3) Magnetron and (4) reactive.

\subsubsection{Chemical Vapor Deposition}

Chemical vapor deposition is the chemical reaction, which transforms gaseous molecules, called precursor, into a solid material in the form of thin film or powder, to produce a nonvolatile solid that deposits atomistically on a suitably placed substrate. Reactions so far used include: reduction of volatile halides; pyrolisis of thermal decomposition of hydrides or halides; disproportionation of halides; and reactions between volatile metal halides or oxides and vaporized elements, leading to the growth of compounds [23]. CVD processes have found increasing applications in solid-state electronic device fabrication, ball bearing and cutting tools production, and the production of rocket engine and nuclear reactor components. The main advantage of CVD methods is the ability to produce a large variety of films and coatings of metals, semiconductors, and compounds possessing high purity and desirable properties. This method also offers the benefits of relatively low cost of the equipment and operating expenses, suitability for both batch and semi-continuous operation, and compatibility with other processing steps. Hence, many varieties of CVD processing have been researched and developed in recent years, including low pressure (LPCVD), plasma-enhanced (PECVD) and laser-enhanced (LECVD) chemical vapor deposition [17]. 


\section{$2.2 \quad$ Film growth}

As the vapor atoms come in contact with a surface they condense and gradually build up a permanent position on the substrate. This is the earliest stage of film formation and termed as the nucleation stage of the film. During this stage, a uniform distribution of small but highly mobile clusters or islands is observed. As the deposition continues, the prior atoms incorporate impinging atoms and the individual islands grow both in size and number. With further deposition the individual islands come very close to each other and start to merge. This process continues until all the islands join to form a continuous thin film on the substrate surface. This collective set of events occurs during the early stages of deposition. Atoms are usually more strongly bound to the substrate than to each other. After formation of the first complete monolayer, a second layer is formed in a similar fashion that covers the previous one and the layer growth mode continues [22], [17].

\subsection{Applications of thin films}

\subsubsection{Electrical and Magnetic}

Deposited films that exhibit ferromagnetism have been extensively developed for computer storage systems. The magnetization vector ensures two states of the film that help data storage in binary notation. Thin films behave as good superconducting systems with well-defined transition temperatures and very small electrical resistance. Film structures also played very important role in the early development of semiconductor device physics, e.g. rectification, photoconduction and luminescence. Resistive and conductive films also have a number of applications. Deposited resistance films can be used for microelectronic circuits, hybrid circuits, electromechanical sensors, chemical sensors and heater elements etc. In a similar way thin conducting films can be used for the elimination of static electricity. Films can be 
made insulating to electrically conducting components, whatever, the main focus is to produce smaller and smaller complex electronic units of extremely high reliability [22], [17], [21], [23].

\subsubsection{Metallurgical and protective coatings}

Several classes of solids, i.e. ionic ceramic oxides, covalent materials, transition metal compounds and metal alloys, are used to form hard, thermal and chemical protective coatings due to their extreme high hardness, very high melting point and resistance to chemical attack. Hard coatings are used to extend the life of cutting tools, dies, punches, and ball bearings by minimizing wear. Thermal coatings find their use in gas turbine engines to improve the performance and life of hot section components. Chemical protective coatings are used in chemical and petroleum industries to protect the underlying material from gaseous, aqueous environment that causes corrosion $[17]$.

\subsubsection{Optical}

Antireflection coatings are used on the lenses of all optical equipment, including cameras, microscopes, binoculars, telescopes and ophthalmic glasses. Systems with wide variety of optical filtering properties can be achieved by using multilayer optical thin films. Thin films are also employed in optoelectronic devices and optical communications, integrated optics and optical recording.

\subsection{Nano-indentation}

Indentation testing is a simple method that involves impressing the surface of a material of interest whose mechanical properties such as elastic modulus and hardness are to be determined with an indenter whose properties are known. The unique characteristic of nano-indentation testing is the length scale involved. Nano-indentation testing involves indenting the specimen usually in nanometers $\left(10^{-9} \mathrm{~m}\right)$ range rather 
than microns $\left(10^{-6} \mathrm{~m}\right)$ or millimeters $\left(10^{-3} \mathrm{~m}\right)$, which is common in a conventional hardness test. Apart from the displacement scale involved, the distinguishing feature of most nano-indentation testing is the indirect measurement of the contact area between the indenter and the specimen, which is one of the most important parameters that needs to be determined correctly to get accurate information about material properties. In conventional indentation tests, the area of contact is calculated by measuring the dimensions of the residual impression left on the specimen surface upon the removal of load. But, in nano-indentation test, the size of the residual impression is so small that it can not be conveniently measured directly. Thus, it is customary to determine the area of contact by measuring the depth of penetration of the indenter into the specimen surface [24]. It is not only hardness that is of interest for materials scientists. Indentation techniques can also be used to calculate elastic modulus, strain-hardening exponent, fracture toughness (for brittle materials), and viscoelastic properties [24].

\subsubsection{Interpretation of load-displacement curve}

The most useful information that is usually extracted from nano-indentation testing is the load-displacement data. A complete nano-indentation test consists of a loading part followed by an unloading part. An indenter, shaped as spherical, conical or cylindrical, is initially placed in contact with the flat surface of the specimen. As the load steadily increases, the indenter penetrates through the surface. Both the load and depth of the penetration are recorded at each load step. After reaching the maximum load the maximum penetration depth is recorded. The initial part of the loading curve usually consists of an initial elastic deformation of the sample, followed by plastic flow, or yield, within the specimen at higher loads. Following the attainment of maximum load, the load is gradually removed as indenter moves out of the specimen at each load step. If any plastic deformation has occurred, the unloading 


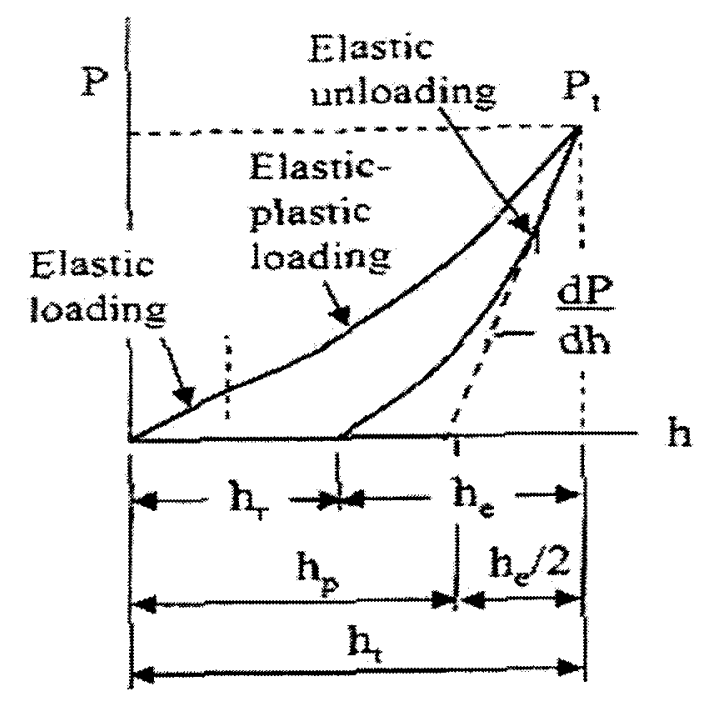

Figure 2.1: Load vs. Displacement curve [24].

load-displacement data follow a different path than that of the loading data until zero applied load is reached. Upon complete removal of load a residual impression made by the indenter is obtained on the specimen surface. Figure 2.1 shows the typical pattern of the load-displacement curve, where, $P_{t}$ and $h_{t}$ are the maximum load and maximum displacement respectively, $h_{r}$ is the depth of the residual impression, $h_{p}$ is the depth of the contact circle and $h_{e}$ is the displacement associated with the elastic recovery during unloading. Detail discussion about measuring different materials properties from load-displacement curve will be presented in the following section.

\subsubsection{Stiffness, Contact area, Modulus and Hardness mea- surement}

The size of the area of contact, determined from known geometry of the indenter, together with the slope of the unloading curve measured at the tangent to the data point at maximum load, lead to a measure of both hardness and elastic modulus of the specimen material [24]. It is thus necessary to know the shape of the indenter and the profile of the unloading curve. In practice, spherical, conical, cylindrical 
or pyramidal indenters are commonly used. The profile of the unloading curve also varies with the geometry of the indenter. For example, the unloading response of the spherical indenter can be expressed as [24]:

$$
P=\frac{4}{3} E^{*} R^{1 / 2} h_{e}^{3 / 2}
$$

where, $\mathrm{P}$ is the indenter load, $h_{e}$ is the elastic displacement of the indenter, $E^{*}$ is defined as the 'reduced modulus', and $\mathrm{R}$ is the relative radius of curvature. To calculate the contact stiffness, modulus, contact area and hardness, the slope of the initial portion of the unloading curve is to be used. This slope of the elastic unloading is calculated by taking derivative of the equations (2.1) with respect to $h_{e}$. For the case of a spherical indenter it will be [24]:

$$
\frac{d P}{d h}=2 E^{*} R^{1 / 2} h_{e}{ }^{1 / 2}
$$

The term $\frac{d P}{d h}$ is sometimes referred to as the contact stiffness and gives the symbol $\mathrm{S}$. For a rigid spherical indenter, Hertz showed that the elastic displacement is given by:

$$
h_{e}=\frac{a^{2}}{R}
$$

where, $a$ is the radius of the circle of contact. Substituting this equation into equation (2.2) gives [24]:

$$
\frac{d P}{d h}=2 E^{*} R^{1 / 2} \frac{a}{R^{\frac{1}{2}}}=2 E^{*} a
$$

Thus, the combined modulus of the system can be determined from the slope of the initial unloading:

$$
E^{*}=\frac{d P}{d h} \frac{1}{2 a}=\frac{1}{2} \frac{d P}{d h} \frac{\sqrt{\pi}}{\sqrt{A}}
$$

where, $A=\pi a^{2}$, the area of contact [24]. Pharr, Oliver and Brotzen have shown that equation (2.5) applies to any indenter that can be described as a body of revolution 
of a smooth function [25]. Hardness, which is defined as the resistance of a material from being deformed permanently while indentation, can be calculated from the following equation [26]:

$$
H=\frac{P_{\max }}{A}
$$

where, $P_{\max }$ is the peak indentation load and A is the projected area of the residual impression. One of the most crucial aspect of nano-indentation testing is to measure the projected area accurately. Several researchers proposed several methods of calculating the projected area. Among those, Pethica et al. suggested a simple method based on measured indentation load-displacement curves and a knowledge of the indenter area function, that is, the cross sectional area of the indenter as a function of the distance from its tip $[27],[26]$. According to their suggestion the final depth gives a better estimation of the contact area than the depth at peak load [26]. Doerner and Nix [28] based on their experimental observation, for some materials, proposed that the nature of the initial portion of the unloading curves (upper one third of the unloading curve), to be linear, that is, the area remains constant while unloading. But Oliver et al. in their work found different observations which suggest that the unloading curves are rarely linear, even in the initial stages of unloading [26]. They also found that unloading contact area changes immediately and continuously as the indenter is withdrawn. An improved technique suggested by Oliver et al. considers the curvature of the unloading curve that is used to determine the depth of penetration after complete removal of load. This depth should be used in conjunction with indenter shape function to measure the contact area at peak load. Figure 2.2 shows a cross section of an indentation used in this analysis. At peak load the displacement $h_{\max }$ can be written as:

$$
h_{\max }=h_{c}+h_{s}
$$




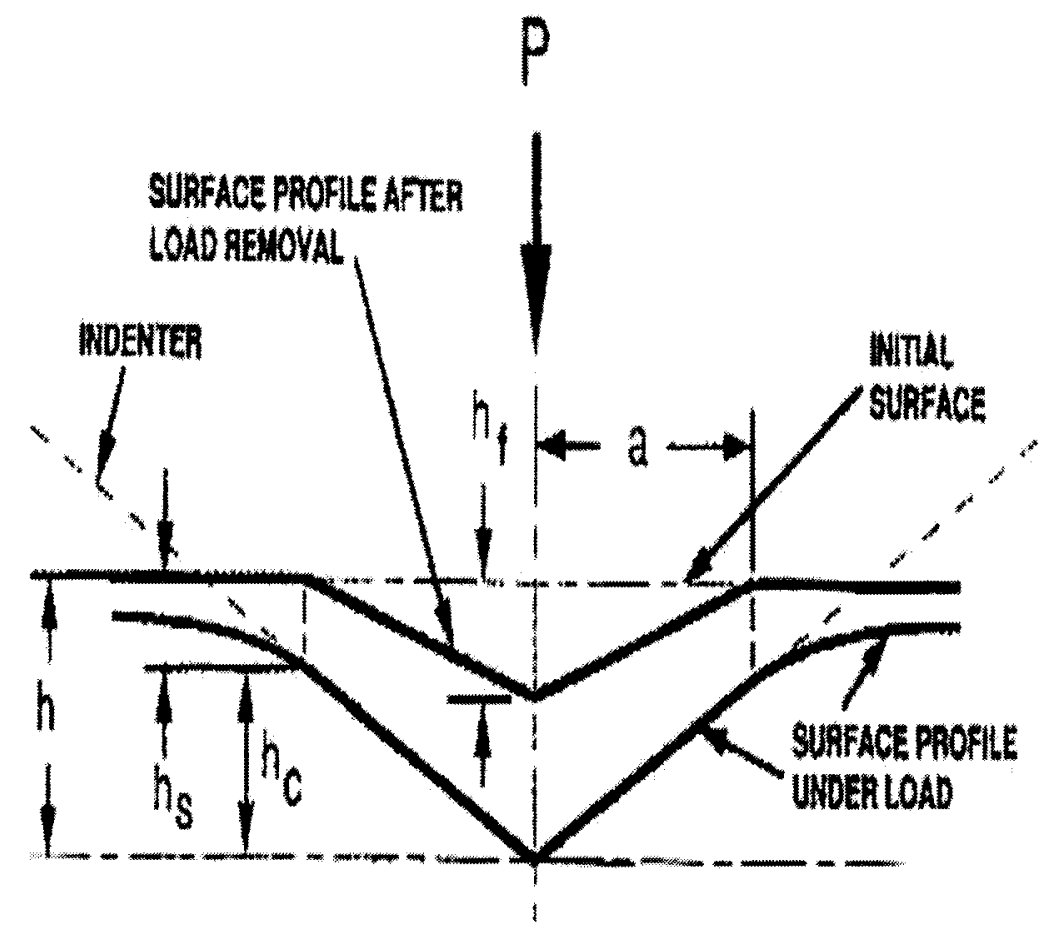

Figure 2.2: A schematic representation of a cross section through an indentation showing various quantities used in this analysis [26].

where, $h_{c}$ is the vertical distance along which contact is made and $h_{s}$ is the displacement of the surface at the perimeter of contact. The value of $h_{\max }$ can be measured experimentally and $h_{s}$ can be obtained from the following equation [26]:

$$
h_{s}=\epsilon \frac{P_{\max }}{S}
$$

where, $\mathrm{S}$ is the stiffness and $P_{\max }$ is the peak load. The value of $\epsilon$ depends upon the geometry of the indenter. Thus, the value of $h_{c}$ can be calculated from the equation (2.7). Following the assumption of Pethica et al. it is assumed that the projected area of the residual impression is a function of the contact depth $h_{c}$ [26],

$$
A=F\left(h_{c}\right)
$$

Though over last few years most of the attention has been focused on the shape of the unloading curves, the mechanical properties of certain materials can not be 
predicted with the help of the existing models. For many stiff hard materials and many inhomogeneous systems the unloading curve fits no existing models particularly well [29]. An alternative approach is to attempt to understand the shape of nano-indentation loading curve [29]. Hainsworth et al. formulated their model for conical and pyramidal indenters and they related the load with depth of penetration by the following equation [29]:

$$
P=E\left(\phi \sqrt{\frac{E}{H}}+\psi \sqrt{\frac{H}{E}}\right)^{-2} \delta^{2}
$$

where, $\delta$ is the indentation depth and $\phi$ and $\psi$ are empirical constants. Now if either $\mathrm{E}$ or $\mathrm{H}$ is known, then the other can be calculated from equation (2.10) [29].

\subsubsection{Factors affecting nano-indentation test data}

The procedures that have been described in the previous section are associated with various errors to measure different material properties in practice. The most serious of these arises while measuring the depth. In addition to this, there are a number of materials related issues that also affect the validity of the results. The most serious of these are the indentation size effect (ISE) and the phenomenon of piling-up and sinking-in [24]. In the following section some of the most commonly encountering sources of error will be reviewed.

\section{Initial penetration depth}

In a nano-indentation test, the depth of penetration has to be measured from the free surface of the specimen. In practice, when the indenter first makes contact with the specimen surface with a very small initial contact load, the depth of penetration is set to be zero. That will establish the depth reference point from which the subsequent measurements will be obtained. This initial contact depth is usually made to be as small as possible by applying the smallest obtainable force of the instrument. However, no matter how small the initial contact force is made there is 


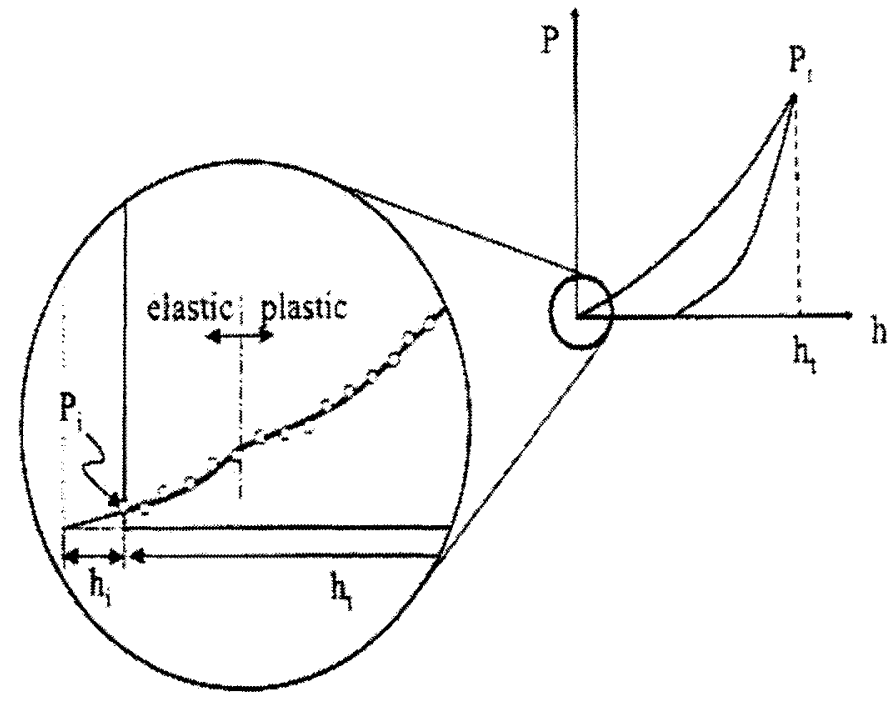

Figure 2.3: Schematic of the initial penetration depth on load vs. displacement data. $P_{i}$ and $h_{i}$ are the initial load and displacement respectively [24].

a corresponding penetration of the indenter beneath the undisturbed specimen free surface, as shown in Figure 2.3. To account for this small initial penetration depth, all subsequent displacement measurements taken from the datum will be increased by this amount. This is done by fitting the load-displacement curve to a smooth polynomial and extrapolating to zero force. The resulting depth offset is then the initial penetration depth and is added to all the depth reading as a correction [24].

\section{Indentation size effect (ISE)}

The indentation size effect (ISE) describes the increase in hardness by several factors by decreasing the contact diameter or indentation depth. Based on similar hardness increase at shallow depth in various matcrials, Stelmashenko et al. [30] and De Guzman et al. [31] explained this behavior of materials in terms of a dislocation density mechanism, Figure 2.4. The mechanism is based on the fact that, at shallow indentation depth the geometrically necessary dislocations (those which form in response to the imposed shape change at the surface) would form over a small 
volume, and result in a high dislocation density beneath the indenter. The high density of dislocations hinders the movement of dislocations and thus increases the hardness of the material. But at large penetration depth, the deformed volume over which they are distributed becomes larger, which results in a decrease in the density of geometrically necessary dislocations. Thus, due to low dislocation density, geometrically necessary dislocations have little effect on hardness at larger penetration depth [31],[30]. They showed the expression for the hardness $H$ in terms of the hardness $H_{o}$ (which would be obtained without the presence of geometrically necessary dislocations or the hardness in the absence of strain gradient):

$$
\frac{H}{H_{o}}=\sqrt{1+\frac{h^{*}}{h}}
$$

where, $h^{*}$ is a length scale constant [31]. Fleck et al. [32], [33] pointed out that indentation size effect for metals can be understood by the large strain gradient at a small scale, which is usually omitted in conventional theories of plasticity. This strain gradient leads to geometrically necessary dislocations that cause enhanced hardness. Following this idea, Nix and Gao [34] developed a model for strain gradient plasticity. Later, based on some experimental observations, Gerberich et al. [35] showed indentation size effect for several materials to be independent of increasing or decreasing strain gradients. They described the theory of strain gradient to be insufficient to explain ISE at depths less than several hundred nanometers. As an alternative model they linked ISE to a ratio between the energy of newly created surface and plastic strain energy dissipation. For indentation depth up to several hundred nanometers, assessment of surface work and volume work can be fundamentally important feature to describe ISE [35].

\section{Piling-up and sinking-in}

In an indentation into an elastic material, the surface of the specimen is typically drawn inwards and downwards underneath the indenter and sinking-in occurs. The 


\section{Geometrically Necessary Dislocations}

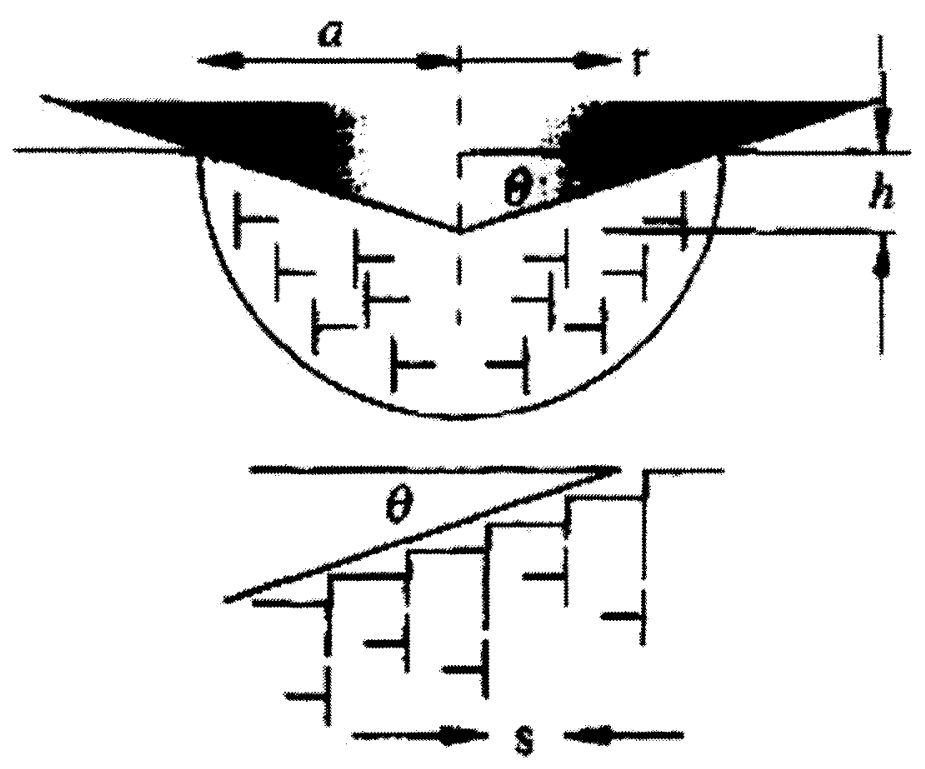

Figure 2.4: Geometrically necessary dislocations created by a rigid conical indenter. Here, $h$ is the depth of indentation, a is the contact radius and $s$ is the spacing between individual slip steps on the indentation surface [34].

plastic zone in this case is typically contained within the boundary of the circle of contact and the elastic deformations that accommodate the volume of the indentation are spread at a greater distance form the indenter. If the surface of the specimen is forced to move outward beside the indenter then piling-up occurs. In this case, the plastic zone meets the surface well outside the radius of the circle of contact [24]. The effect of piling-up and sinking-in on the contact area is shown in Figure 2.5. The existence of piling-up and sinking-in can have a detrimental effect on the determination of the area. A correction factor can be applied to determine the contact area correctly. Beside the above mentioned factors, indenter geometry, surface roughness, residual stress, thermal drift etc. also play a crucial role which affects nano-indentation test data. Moreover, several researchers have made experimental observations to determine the effects of tip radius [36], work hardening [37], and surface steps [38] on nano-indentation property measurement of different materials. 
(a)
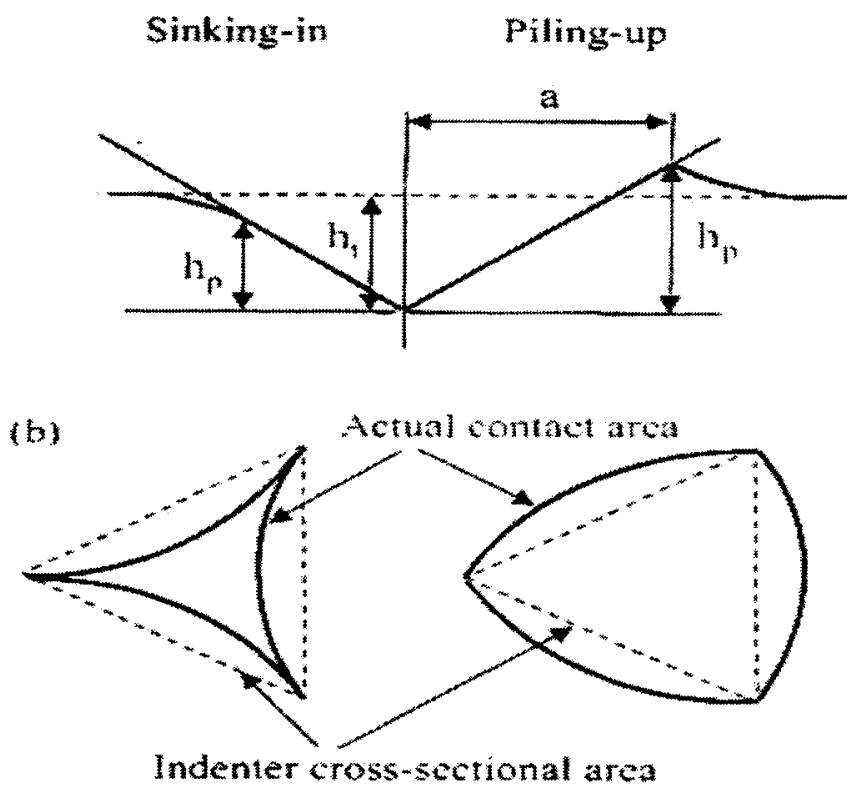

Figure 2.5: Effect of sinking-in and pile-up on the actual contact area. (a)Crosssectional view (b)plan view [24].

\subsection{Hardening theories at the nanometer scale}

Experimental results show that a nanolayered structure made of two kinds of metals strengthens dramatically as the layer thickness is reduced. Several theoretical models have been postulated based on a single dislocation gliding along one layer and many dislocations piling up at the boundary between the layer interfaces.

\subsubsection{Orowan Model:}

Orowan model [39] states that while moving along the softer phase the movement of a dislocation is blocked at the interface of the harder phase and the first deviation from linear elastic behavior occurs when a dislocation can be curved into a semicircle within a layer of the softer phase [9], Figure 2.6. The strength $\sigma^{*}$ of the multilayers scales with $\frac{\ln h}{h}$, where, $\mathrm{h}$ is the layer thickness. As the stress rises above this point, 


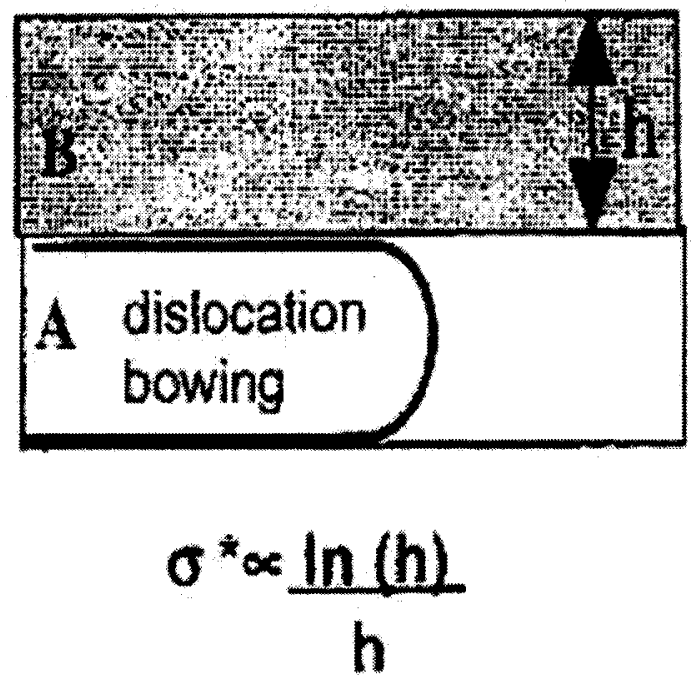

Figure 2.6: Single-dislocation based Orowan model that contributes strengthening in nanoscale multilayers [40].

an increasing fraction of the load is transferred to the harder phase. The harder phase remains elastic until it either fractures or yields [9].

\subsubsection{Hall-Petch Model:}

It is well known that the Hall-Petch equation describes the relationship between yield strength and grain size [41]. According to this model, the yield strength scales with the inverse square root of the relevant microstructural length scale (grain size or interlamellar spacing or bilayer period). Since hardness scales linearly with strength (typically, hardness is a factor of 3 higher than the flow stress) the Hall-Petch model is also applicable in interpreting hardness increases with decreasing microstructural length scales [40].

$$
\sigma=\sigma_{0}+k_{\sigma} d^{-1 / 2}
$$

or

$$
H=H_{0}+k_{h} d^{-1 / 2}
$$




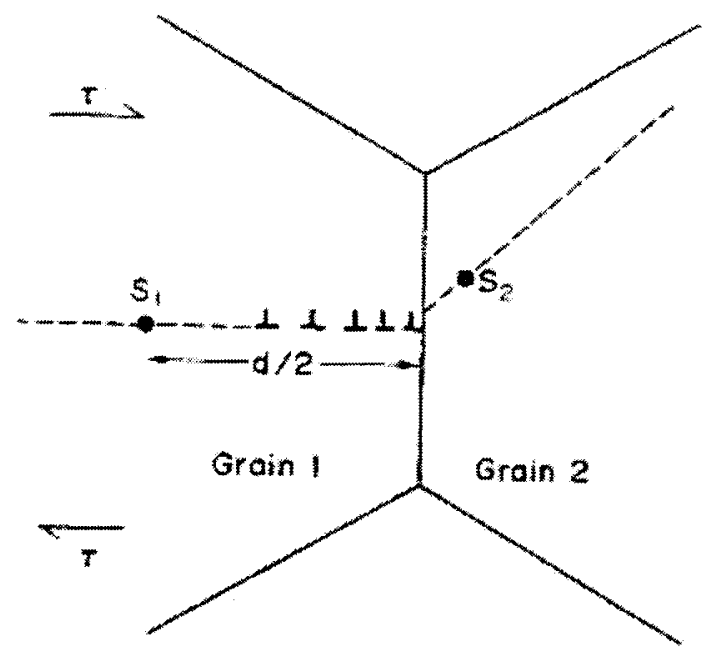

Figure 2.7: Schematic illustration of a pile-up formed in grain 1 under an applied shear stress $\tau . S_{2}$ is the source in grain 2. The trace of preferred slip plane is shown by dashed lines [42].

where, $d$ is the length scale. The view of this model is that yield occurs when the leading dislocation in a pile-up overcomes the barrier stress at an interface [9] or the pile up at a grain boundary in one grain can generate sufficiently large stresses to operate sources in an adjacent grain at the yield stress, as shown in Figure 2.7 [42]. However, several studies on nanocrystalline materials have confirmed that at a length scale of a few tens of nanometers to $100 \mathrm{~nm}$, the Hall-Petch effect is often seen to cease or even to reverse, which is known as the 'Reverse Hall-Petch effect'. Figure 2.8 shows the Hall-Petch plot, that is Hardness vs. $h^{-0.5}$ for several physically vapordeposited Cu-based multilayered systems. This figure shows linear fit at larger h, which is consistent with the Hall-Petch model, but at lower h, the Hall-Petch model breaks down [40]. Several mechanisms have been proposed for this reverse Hall-Petch effect: increased porosity at small grain sizes [43], suppression of dislocation pile-ups [41], dislocation motion through multiple grains [44], sliding in the grain boundaries [45], and enhanced diffusional creep in the grain boundaries [46]. Schiotz et al. [47], based on their observation, described the occurrence of plastic deformation due to a 
large number of 'sliding' events of atomic planes at the grain boundaries, with only a minor part being caused by dislocation activity in the grains. Softening that they observed was due to the larger fraction of atoms at the grain boundaries [47]. Nieh et al. [41] proposed a grain boundary relaxation mechanism that could lead to a decrease in strength at extremely fine grain sizes [41]. Based on a dislocation pile-up model, as the grain size of the material decreases, there arrives a point at which each individual grain will no longer be able to support more than one dislocation; at this point the Hall-Petch relation will no longer hold. From another point of view, when the grain size approaches zero, the material essentially becomes amorphous. The grain boundary strengthening effect will then disappear [41]. Nieh et al. calculated the grain size based on an estimation of repulsive force per unit length between two edge dislocations at which the strength reach a maximum value. The equilibrium distance between two edge dislocations can be expressed as:

$$
l_{c}=\frac{3 G b}{\pi(1-\nu) H}
$$

In principal when the grain size, $\mathrm{d}$, is smaller than $l_{c}$, there will be no dislocation pile-ups, and the HP relation will break down [41]. The evaluation of the strength of metallic multilayers as a function of layer thickness may be divided into four stages [40]:

- Stage $I$ (Hall-Petch regime). This is observed down to length scales of a few tens of nanometers to $100 \mathrm{~nm} ; \sigma^{*} \propto h^{-1 / 2}$.

- Stage $I I . \sigma^{*} \propto h^{-a}$, where $a \neq 0.5$. This is typically observed for layer thickness range of a few tens to a few nanometers.

- Stage III. A peak or saturation in strength is observed when the layer thickness is a couple of to a few nanometers.

- Stage $I V$. Strength decreases with decreasing layer thickness, typically for h below a couple of nanometers. 


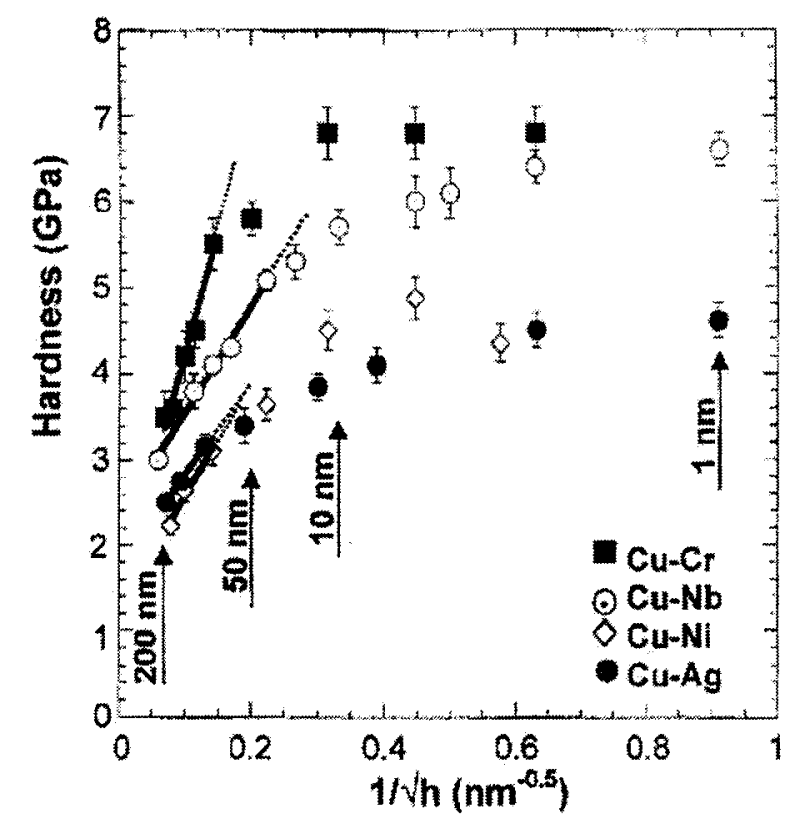

Figure 2.8: Hall-Petch plot for several Cu-based multilayers. Linear fit $(-)$ at larger $h$ consistent with the Hall-Petch model. Al lower $h$, the Hall-Petch model (....) breaks down [40].

Neither of these models is a complete theory of yield for multilayer systems. The Orowan model neglects the effect of coherency and thermal expansion mismatch stresses and it focuses on the yield of just one phase. The Hall-Petch model pays no attention to the operation of dislocation sources [9]. Moreover, the mechanism behind the Hall-Petch model is not directly applicable to the multilayers [19], rather it is more applicable to the grain size effect in polycrystalline materials. In order to improve either model, or to develop a combined model, a key requirement is to clearly understand the barrier strength $\sigma^{*}$ of the multilayer system. In order to do this, a detailed idea about dislocation-interface interaction is necessary.

\subsubsection{Dislocation-Interface Interaction}

In the classical approximation the blocking strength of an interface is attributed to modulus mismatch, lattice parameter mismatch, chemical or gamma surface mismatch, and slip-plane mismatch between two adjacent layers. The interaction of 
dislocations with the interface due to these mismatches will be presented in the following sections.

\section{Modulus interaction: Image Effect}

Due to the difference in shear modulus between two adjacent layers, the strain energy per unit length of a dislocation is generally lower in one lamella than in its neighbor, which induces a force between a dislocation and its image in the interface. Kochler calculated the additional stress (also known as Koehler barrier) which is required to move a screw dislocation from a soft lamella into a hard lamella against its own elastic image in the interface. The result is [48]:

$$
\tau^{*}=\frac{G_{1}\left(G_{2}-G_{1}\right) b}{4 \pi\left(G_{2}+G_{1}\right) h}
$$

where, $h$ is the distance of the dislocation from the interface, $b$ is the Burgers vector, and $G_{1}$ and $G_{2}$ are the shear modulus of adjacent layers. Since the value of $\tau^{*}$ approaches infinity as $\mathrm{h}$ approaches zero, Equation (2.15) is only valid for $h>2 b$, i.e. it does not consider the dislocation core effects. Atomistic simulation by Rao et al. [9] shows that Koehler stress is an effective barrier to dislocation motion in $\mathrm{Cu}-\mathrm{Ni}$ bilayer system, but for $\mathrm{Cu}-\mathrm{Ni}$ multilayered system Koehler stress usually decreases with wavelength of the multilayer due to the interaction of gliding dislocations with more than one lamellar interface. Based on Peierls dislocation model, Pacheco and Mura [49] derived the shear stress acting on the dislocation located at a distance $\mathrm{x}$ from a perfectly abrupt interface, which is valid for all $\mathrm{x}$ including the dislocation core:

$$
\tau=\frac{2 \alpha\left(G_{B}-G_{A}\right) \sin \theta}{\pi}\left(\frac{b^{2}}{4 x^{2}+b^{2}}+\frac{b}{2 x} \tan ^{-1} \frac{2 x}{b}\right)
$$

where, $b$ is the magnitude of the Burger's vector and $\alpha$ is $\frac{1}{4 \pi}$ for screw dislocation and

$\frac{1-\nu}{4 \pi}$ for edge dislocation. To compare this result with hardness data, it is necessary to first convert the shear stress to a yield stress using Schmidt's law, $\sigma=\tau / m$, 
where $\mathrm{m}$ is the Taylor factor, and hardness can be estimated from the yield stress using $H=3 \sigma[4]$. Using this formula Chu and Barnett [19] showed that the yield strength or hardness increases with increasing supcrlattice period before reaching a saturation value for different interface width.

\section{Dislocation glide within individual layers}

When the layer thickness is very large, dislocations will move large distances without encountering an interface and will not experience significant image effects. Thus, there must have an optimal layer thickness to inhibit dislocation motion that results in maximum hardness. Sevillano [50] modelled the critical stress necessary to propagate dislocations within individual layers of a multilayer. According to his model, the critical shear stress required to move a pre-existing dislocation loop that is confined within a thin layer is given by [4]:

$$
\tau_{L A}=\tau_{O A}+\frac{2 \alpha G_{A} b \sin \theta}{t_{A}} \ln \left(\frac{t_{A}}{b \sin \theta}\right)
$$

where, $\tau_{O A}$ is the critical shear stress required to move the dislocation loop in an infinitely thick layer of material A. He also noted that if there are not sufficient dislocations present, a larger shear stress is needed to first generate the dislocations within the layer. This shear stress is given by [4]:

$$
\tau_{U A}=\tau_{O A}+\frac{4 \alpha G_{A} b \sin \theta}{t_{A}} \ln \left(\frac{t_{A}}{2 b \sin \theta}\right)
$$

Chu and Barnett [19] combined the theory by Sevillano for dislocation motion within an individual layer with the model for image effect to obtain an overall picture of the hardness behavior of a multilayer system. According to their model the overall strength of a multilayer system, $\sigma_{t o t}$, can be expressed as:

$$
\sigma_{t o t}=\sigma_{O}+\frac{2 \alpha b \cos \theta}{m \Lambda}\left[G_{2}{ }^{0} \ln \left(\frac{l_{2}}{b \cos \theta}\right)+G_{1}{ }^{0} \ln \left(\frac{l_{1}}{b \cos \theta}\right)\right]
$$

where, $G_{1}{ }^{0}$ and $G_{2}{ }^{0}$ are the shear modulus of pure materials 1 and $2, \Lambda$ is the bilayer period, $m$ is the Taylor factor, $b$ is the burgers vector. The model predictions have 


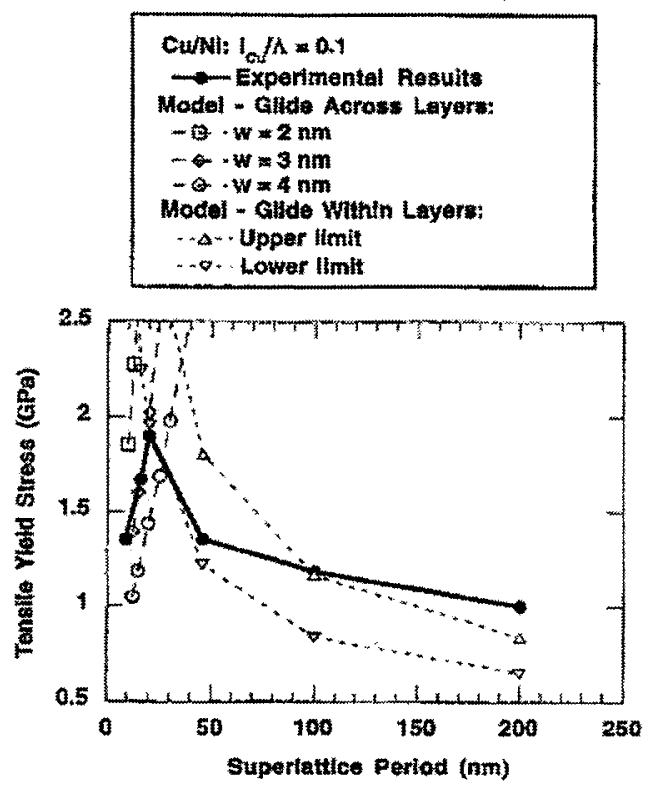

Figure 2.9: Experimental tensile yield stress vs. superlattice period for $\mathrm{Cu} / \mathrm{Ni}$ superlattices compared with model predictions for dislocation glide across and within layers [19].

been compared with the experimental observations for different metallic, nitride and metal/nitride multilayers and have shown good agreement [19],[4], [5],[2], [20],[51]. Figure 2.9 and Figure 2.10 show the comparison between the experimental results and the model predictions for yield stress and hardness variation with superlattice period for $\mathrm{Cu} / \mathrm{Ni}$ and $\mathrm{TiN} / \mathrm{NbN}$ superlattices.

\section{Size Interaction}

In multilayers, the lattice parameter mismatch between the adjacent layers is usually taken up by misfit dislocations and coherency strain. When the lattice mismatch is comparatively small (below the coherency wavelength limit $\left(\lambda_{c}\right)$ ), then the adjacent layers form a strained structure and the total misfit is accommodated by the coherency strain. But larger mismatch of lattice parameters (above the coherency wavelength limit $\left(\lambda_{c}\right)$ ) relaxes the strained structure gradually, and a part of the misfit is taken up by an array of misfit dislocations at the interface and rast of the 


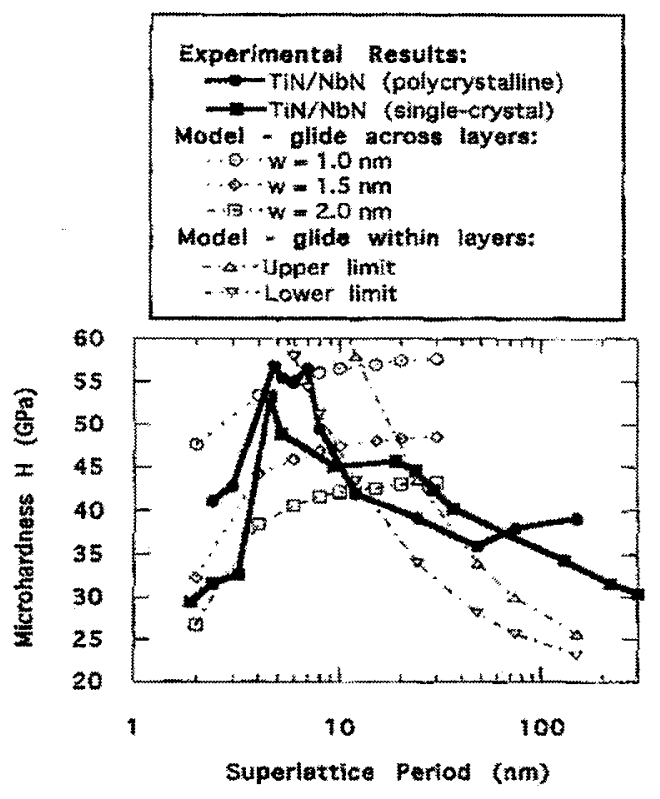

Figure 2.10: Experimental hardness vs. superlattice period for TiN/NbN superlattices compared with model predictions for dislocation glide across and within layers [19].

misfit is taken up by the coherency strain. This results in an increased density of misfit dislocation with increasing lattice mismatch between adjacent lamellae. These misfit dislocations act as obstacles to glide dislocation movement, at or near to the interface. In general this barrier is expected to increase if the spacing between the misfit dislocations decreases. The blocking strength of misfit dislocations to glide dislocation movement can be estimated as [9]-

$$
\tau_{d}= \begin{cases}\alpha \mu^{\frac{\delta_{a}}{a}-b} & \text { for } \lambda>\lambda_{c}, \\ 0 & \text { for } \lambda<\lambda_{c}\end{cases}
$$

where, $\frac{\delta a}{a}$ is the misfit of the lattice parameter, $\alpha=0.5, b$ is the Burgers vector and $\lambda$ is the wavelength. Rao et al. [9] showed that the misfit dislocations are an effective barrier to the glide dislocations for long wave lengths in the $\mathrm{Cu}-\mathrm{Ni}$ multilayer system, but the effect decreases gradually with multilayer wavelength. 


\section{Chemical Interaction}

'Chemical' or 'gamma surface' mismatch between the lamellae changes the dislocation core energy as it approaches the interface. Both the self-energy of the dislocation and the interaction energy between the Shockley partials continuously vary as the dislocation moves from one lamella to next due to modulus mismatch between the lamellae. However, the stacking fault energy changes abruptly as the leading partial moves into the next lamella, leading to a Koehler stress as discussed before. The chemical component of the blocking strength thus depends upon interface properties, in this case the stacking fault energy of the interface [9].

\section{Slip plane mismatch}

For a twinned interface where slip planes are not continuous, screw dislocations have to cross slip for transmission of slip across the interface. As a result, the blocking strength of such interfaces is expected to be substantially larger [9].

\subsection{Computational approach}

As the field of computational material science expands, computer modeling and simulation are becoming an established tool and an integral part of materials research and design. In recent years, the advent of ever more powerful and massively parallel computers has enabled the development of new concepts and algorithms for the computational modeling of materials [52]. The motivations for computer simulations of physical systems are many fold. One of the main motivations is that the computer simulation approach allows one to apply theory to study complex systems and gain insight into their behavior. Indeed, the complexity can go far beyond the reach of present analytical methods. There is yet another feature - computer simulations can fill the gap between theory and experiment. Some quantities or behaviors may be impossible or difficult to measure by experiment. With computer simulation such 
quantities can be computed. The laboratory experiment tells us what happens, whereas, theory combined with computation, allows us to find out why it happens and hopefully also to predict what will happen under similar circumstances. Moreover, by comparing the results with experiment, the validity of the model can also be ensured [53]. Other important aspects of computer simulation are the stability, accuracy and efficiency of the solution, which are of utmost importance for solving numerical algorithms. The explanatory, reliable and predictive capability of computer simulations can explain the ever-increasing impact of computation on modern materials science [54].

\subsubsection{Atomistic modeling scheme}

Atomistic simulations are playing an increasingly prominent role in materials science. At the atomic scale, defects such as dislocations, their core structures and mechanisms of their nucleation and motion play an important role in affecting the macroscopic material behavior. Conventional theoretical modelling and computer simulations such as those based on continuum approaches can not reveal the effects of such defects. Thus, those models must be supplemented with atomistic models to characterize materials behavior [13]. Predictions resulting from this atomic-level understanding are proving increasingly accurate and useful. Consequently, the field of atomistic simulation is gaining ground as an indispensable partner in materials research [12].

\section{Interatomic Potentials}

With the advancement of atomistic simulations, theoretical descriptions of interatomic potential functions are also necessary to describe atomic interactions. Interatomic potential functions play the most important role in every atomistic simulation. The accuracy and complexity of the potential determine the quality of the simulation results and the amount of computer time required [12]. 
Density functional theory (DFT) is one of the earliest methods wherein simulations were aimed towards the solution of the complex quantum many-body Schroedinger equation of the atomic system using numerical algorithms. Though the DFT method provides a highly accurate description of the quantum mechanical behavior of materials, the system sizes are still limited to only about a few hundred atoms. Moreover, the simulation time required is often many days of CPU time. Thus, it is essentially impossible to compute the mechanical response of a system of realistic dimension with this approach [55]. To get rid of this problem, physicists have developed more approximate descriptions of the interaction between particles. These semi-empirical models start by assuming a simplified form for the atomic interactions [56]. Substitution of a simpler (faster) potential allows one to obtain quick qualitative results; to scan many cases to obtain trends; to investigate longer time scales; and to model a larger system [12]. The simplest of such semi-empirical models is the pair potential, where the energy, $E_{\text {tot }}$ of an arbitrary arrangement of $N$ atoms is written as

$$
E_{t o t}=E_{0}+\frac{1}{2} \sum_{i}^{N} \sum_{j \neq i}^{N} \Phi_{i j}\left(r_{i j}\right) .
$$

Here $E_{0}$ is a constant, volumetric energy, $\Phi_{i j}$ is an energy potential that depends on the atomic species of both atoms $i$ and $j$ and $r_{i j}$ is the distance between the $i^{\text {th }}$ and $j^{\text {th }}$ atoms. The pair potential is limited in its applicability, both with respect to the materials that it can represent as well as the range of deformations the material may undergo [56]. Daw et al. derived a new method, the Embedded-Atom Method (EAM), based on density functional theory to calculate ground-state properties of realistic metal systems [57]. In EAM, the energy is expressed as a sum of individual atom energies and the total potential cnergy, $E_{t o t}$, of a configuration of atoms is written as [57]

$$
E_{t o t}=\frac{1}{2} \sum_{i} \sum_{j(\neq i)} \Phi_{i j}\left(r_{i j}\right)+\sum_{i} F_{i}\left(\bar{\rho}_{i}\right)
$$


In this expression, $\Phi_{i j}$ is a pair potential evaluated for the interatomic distances $r_{i j}$, $\bar{\rho}_{i}$ is the host electron density at atomic position $i$ due to neighboring atoms, and $F_{i}$ is the embedding energy which is physically interpreted as the energy associated with placing an isolated atom in a uniform electron gas of density $\bar{\rho}_{i}[56]$. The function $\bar{\rho}_{i}$ is found by linearly superposing the electron density from all neighboring atoms via the expression

$$
\bar{\rho}_{i}=\sum_{j(\neq i)} \rho_{j}\left(r_{i j}\right)
$$

where $\rho_{j}$ is the spherically averaged electron density field of an isolated atom. In this and the previous equation, the sums on $j$ are over all atoms within some cutoff radius, $r_{c u t}$ of atom $i$. This cutoff generally includes the first 2 or 3 neighbor distances in the crystal, beyond which it is assumed that atoms do not interact [56]. The ground-state properties, e.g. lattice constant, elastic constants, sublimation energy and vacancy-formation energy of the solid can be calculated from Equation (2.22) $[57]$.

\section{Molecular Dynamics(MD) method}

Molecular dynamics is a popular simulation method that permits a computation scale less than or equal to giga-atoms and a simulation period shorter than nano seconds [58]. In MD simulations, a physical system of $\mathrm{N}$ atoms is represented by a set of atomic coordinates, $\left\{x_{i} \mid i=1,2, \ldots, N\right\}$ and an algorithm is used to find a numerical solution of a set of coupled first-order ordinary differential equation, given by the Hamiltonian formulation of Newton's second law [13]

$$
m_{i} \frac{d^{2}}{d t^{2}} x_{i}=\frac{-\partial}{\partial x_{i}} V
$$

where, $m_{i}$ is the mass of the $i^{\text {th }}$ atom and $\mathrm{V}$ is the interatomic potential. The equation of motion is numerically integrated forward, with a finite time step [55]. During the simulations, the response of a material subjected to an external force is 
evaluated by following the dynamics of every atom in the simulation sample. The MD simulation comprises two phases: initial relaxation and loading. In the first phase, atoms are positioned according to an assumed lattice sites and their velocities are randomized to satisfy the Maxwell-Boltzman distribution at prescribed temperature. In order to reach the equilibrium state, the atoms are relaxed according to Equation (2.24) in the absence of any external force. In the second phase, an appropriate loading is applied by tiny increments. Thousands of relaxation steps will be engaged after each loading increment [58].

\subsubsection{Multiscale modeling scheme}

It has clearly been understood that the materials properties are profoundly affected by the interesting phenomena occurring at different length scales. At the atomic (angstrom) scale, understanding of atomic interactions is necessary to characterize materials behavior. At nanometer scale, defects such as dislocations, their core structures and mechanisms of their nucleation and motion control the mechanical behavior, while at scales of the micrometer or more, large collections of such defects including grain boundaries, dislocation pile-ups at grain boundaries and other microstructural features, govern materials properties. However at an engineering scale, forces arising from macroscopic stresses and/or temperature gradients may be the controlling elements of materials performance [52]. Fully atomistic simulations are essential for resolving the atomic scale phenomena, but they cannot be used to model entire systems on the micrometer scale, since computations are limited by reasonable memory requirements and simulation times. At the present state of the art, the length scale amenable to atomistic modeling is well below the typical grain size, which is required to model realistic microstructures [16]. In recognition of this limitation, the field of multi-scale materials modeling has emerged, whereby atomistic modeling is used in conjunction with larger scale models to predict the 
performance and behavior of complex materials across all relevant length and time scales [52]. Multiscale modeling methods fall under one of the two possible categories. One approach is a hierarchical scheme, wherein modeling is done separately at different length scales and the behavior of the system at the smaller scales is used as a source of parameters for the larger scales [16]. Such sequential modeling approaches are usually effective when material behavior at different scales has its own distinct characteristics [52]. The second approach is the hybrid scheme, in which some regions of a problem are treated by a fully atomistic approach whereas the other regions are solved by continuum approach, thus considering the material behavior at different scales concurrently in a combined model [52]. In this section a brief review of several methods will be presented. Detail discussion on the CADD model, which is the focus of this thesis, will be presented in Chapter 3.

\section{Discrete Dislocation modeling}

The Discrete Dislocation (DD) method presents a framework for solving boundary value problems wherein dislocations are treated as continuum entities moving in fields determined by elasticity [59], [60], [61], [62], [16]. In the DD approach, a continuum problem is divided into two complementary problems, shown in Figure 2.11. Problem I consists of dislocations in an infinite elastic continuum and is solved by superposition of the elastic fields due to the discrete dislocations. Problem II consists of a linear elastic continuum with no dislocations that enforces the boundary condition across the internal boundaries. All discontinuities and singularities of the dislocations are contained in the elastic fields due to the discrete dislocations, so the fields of problem II, denoted as image fields, are smooth and can be solved by standard numerical techniques, e.g. finite element method [14]. More detailed discussion on the discrete dislocation method will be presented in Chapter 3. Thus, the long range interactions between the defects are modeled accurately. The displacement $(u)$ and stress field $(\sigma)$ can be written by superposition of the two fields mentioned 


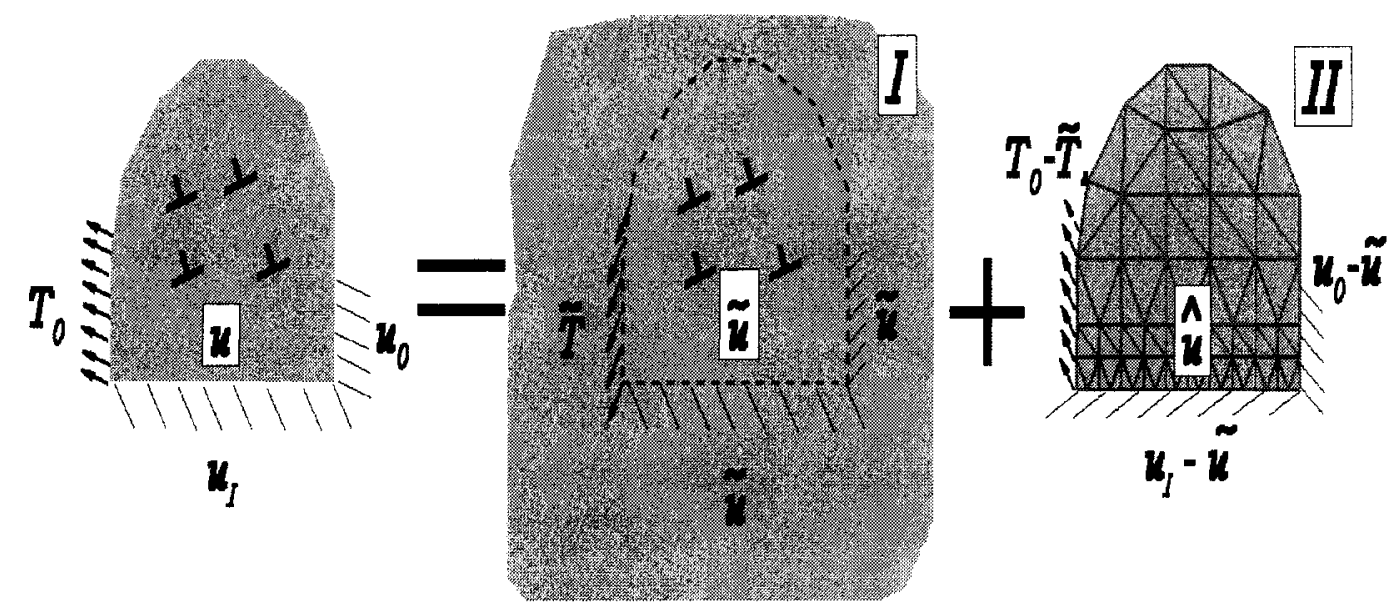

Figure 2.11: Schematic illustration of the solution procedure of the discrete dislocation method [16].

before:

$$
\begin{array}{r}
u_{i}=\tilde{u}_{i}+\hat{u}_{i} \\
\sigma_{i j}=\tilde{\sigma}_{i j}+\hat{\sigma}_{i j}
\end{array}
$$

The () fields are the sum of the fields of the individual dislocations, in their current configuration and the () fields represent the image fields that correct for the actual boundary conditions on the boundary. Short range dislocation interactions are governed by a set of constitutive rules, for instance, the conditions required for dislocation nucleation, the minimum dislocation separation for annihilation, the strength of dislocation junctions, the dislocation mobility, and dislocation/obstacle interaction [59], [16]. Suitable values for the parameters in these rules can be obtained through atomistic simulations.

\section{Quasicontinuum (QC) method}

Instead of identifying atomistic and continuum region, the QC method [63],[64] defines two types of atoms: 'non-local representative atoms' and 'local representative 
atoms'. In practice, these two atoms are essentially equivalent to the real atoms of the fully atomistic regions and the FE nodes of the continuum elements respectively [65]. The transition region between non-local and local representative atoms in the QC model is depicted in Figure 2.12. The pad atom positions are dictated by

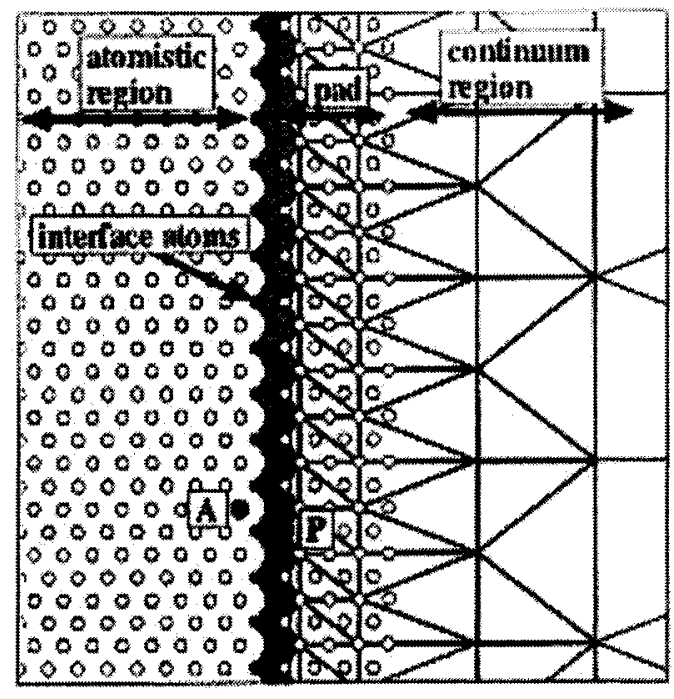

Figure 2.12: The atomistic/continuum region for the QC method [65].

interpolation from the FE nodal positions. The pad atom energies are not included in the total energy calculation, but the energies of the real and interface atoms include their interactions with the pad atoms. The interface atoms act as real atoms during the atomistic energy calculation but are also FE nodes of the continuum. Thus, the energies of the continuum elements adjacent to the interface (i.e. elements that have nodes corresponding to the interface atoms as shown in grey in the figure) are weighted differently in the total potential energy sum to avoid overestimation of energies of the interface atoms. The total potential energy of the QC model is then obtained by summing the energies of all atoms in the atomistic region and at the interface and all elements in the continuum domain as [65]-

$$
E_{Q C}=\sum_{i \in(A, I)} E_{i}\left(\boldsymbol{r}_{A}, \boldsymbol{r}_{I}, \boldsymbol{r}_{P}\right)+\sum_{\mu} \omega_{\mu} E_{\mu}
$$


where, the first term represents the total energy of the atomistic region by summing the energies of the real atoms and the interface atoms $[i \in(A, I)], \boldsymbol{r}_{A}, \boldsymbol{r}_{I}, \boldsymbol{r}_{P}$ are the position vectors of real, interface and pad atoms respectively, the second term gives the total energy of the continuum region by summing the energies of individual elements $E_{\mu}$ in the continuum region. $\omega_{\mu}$ is the weight function and it implies that elements that touch the interface contribute a fraction $\omega_{\mu}$ of their energy, while all other elements contribute their entire encrgy to the sum. The $\omega_{\mu}$ depends on the shape of the element, but always satisfy $0<\omega_{\mu}<1[65]$.

\section{Finite Element and Atomistic (FEAt) method}

The FEAt model [66], is the only model that attempts to solve the problem of a local/non-local mismatch by introducing a non-local continuum region near the atomistic/continuum interface. The transition region for the FEAt model is shown in Figure 2.13, where the grey elements are elements in the pad region. In the FEAt model, the pad atoms directly overlap the continuum nodes and it extends to twice the cut-off radius of the EAM interatomic potentials. In this way, the FE region is resolved down to the atomic-scale in the pad region, and atoms in the atomistic region have their full compliment of their neighbors. The FEAt model does not make use of a unified energy function for the entire coupled problem [65]. The energy in the elements in the FEAt model is defined in two different ways. Outside of the pad region, non-linear elasticity is used. In an effort to mitigate the effects of the sharp transition from a non-local atomistic region to a local continuum, the FEAt model also uses a non-local continuum formulation to describe the energetics of the elements in the pad region. The energy of the continuum region in the FEAt is then found by summing the energy the local and non-local FEs [65]-

$$
E_{F E A t}^{c}=\sum_{\mu \in P} E_{\mu}^{(\text {nonloc })}\left(\boldsymbol{r}_{I}, \boldsymbol{r}_{P}\right)+\sum_{\mu \in P} E_{\mu}^{(\text {nonlin })}
$$


The energy of the atomistic region is found by the usual sum over atoms, including the pad atoms, as [65]-

$$
E_{F E A t}^{a}=\sum_{i \in A, I, P} E_{i}\left(\boldsymbol{r}_{A}, \boldsymbol{r}_{I}, \boldsymbol{r}_{P}\right)
$$

where the positions of the pad atoms provide the neighbor environment for all the atoms in the sum.

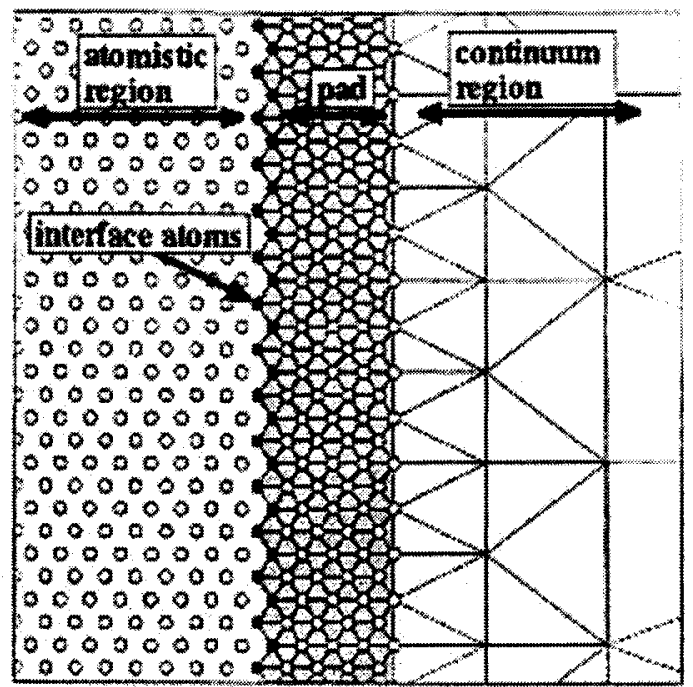

Figure 2.13: The atomistic/continuum region for the FEAt method [65].

\section{Concurrent Length Scale (CLS) method}

In the CLS method [67], the model is designed in such a way that all of the pad atoms are always in direct correspondence with nodes of finite elements in the continuum region, as in the FEAt model. Also, as in the QC method, elements that are in contact with the interface contribute less than their full energy to the total potential energy of the system, as thus these are shaded in gray in the Figure 2.14. In the continuum region all the finite elements are modeled as linear elastic. The atomistic energy of the CLS method is described by the potentials with two- and three-body interactions. Rather than considering atom-by-atom basis interactions, the CLS method was formulated in terms of the energy of each bond in the system. 
Specifically, each two- or three-body bond contributes its full energy to the system if all of the participating atoms in the bond are in the atomistic region or on the interface. If the bond includes one or more atoms from the pad, the contribution is scaled by a factor of $1 / 2$ [65]. Examples of bonds that contribute fully (dark lines) and partially (dash lines) are shown in Figure 2.14 [65].

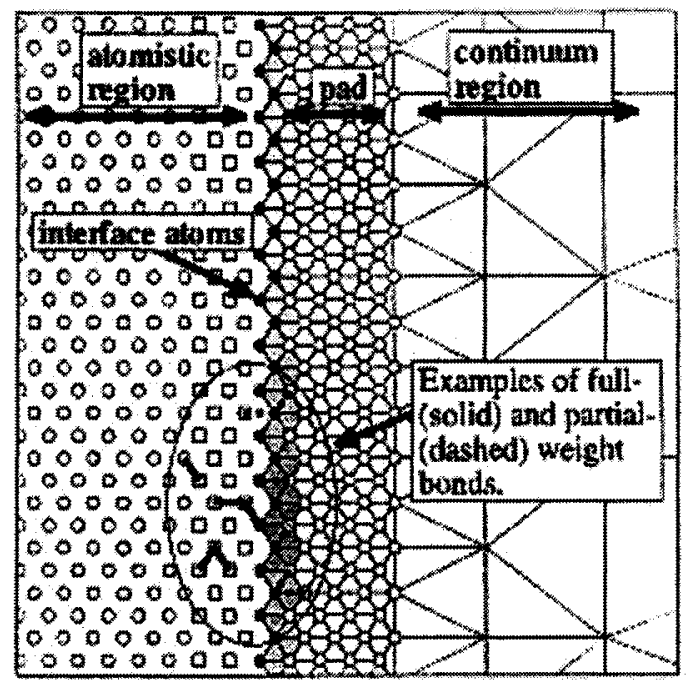

Figure 2.14: The atomistic/continuum region for the CLS method [65]. 


\section{Chapter 3}

\section{Coupled Atomistic and Discrete Dislocation (CADD) Method}

We have discussed in the previous section that it is advantageous to implement multiscale modelling schemes in solving problems occurring at several length and/or time scales, but most of the existing methods have some limitations associated with them. For example, the FEAt method [66] and the CLS approach [67] are unable to support continuum dislocations in the finite element region. Moreover, the size of the atomistic region can not be easily adapted during the simulation. The quasicontinuum (QC) method of Tadmor et al. also do not allow for the continuum description of a dislocation, and therefore, every dislocation in the QC model requires a fully atomistic description around its core and slip plane [16]. Hence, computational cost becomes enormous while handling large number of dislocations. The Coupled atomistic and discrete dislocation (CADD) model is a new hybrid scheme that extends the capabilities of the existing models by incorporating some unique features. Like most other existing hybrid models (i.c., FEAt [66], CLS [67], QC method [64]), the CADD method also begins by separating the physical problem into spatial regions that are modelled either by a fully atomistic description or by continuum finite elements. But the most unique characteristic of the CADD method is its ability to accommodate discrete dislocations in the continuum region as well as in the atomistic region. Like other methods the CADD model successfully reduces the computational memory 
and time from what would be required to perform the same simulations using a fully atomistic model. Moreover, the CADD framework can detect dislocations near the atomic/continuum interface and pass them across the interface efficiently [14],[16]. The CADD method consists of four essential components: the atomistic model, the continuum model, the coupling between these two regions and the method for detecting and passing dislocations between these two regions. The first two components, the atomistics and continuum framework, are simply taken from the well-developed methods found in the literature. The atomistic framework is adopted from the embedded atom method (EAM) (see section 2.6.1) and the continuum problem can be solved using the DD framework developed by van der Giessen and Needleman [59] as discussed in section 2.6.2. The details of the coupling between these two regions and the detection and passing of dislocation across the interface are the new additions in this model [16].

A body $\Omega$, shown schematically in the top left of Figure 3.1, is imagined to be divided into an atomistic region $\Omega_{A}$ and a continuum region $\Omega_{C}$ joined at an interface $\partial \Omega_{I}$ defined by a row of atoms. The body is subjected to traction $\boldsymbol{T}=\boldsymbol{T}_{0}$ on $\partial \Omega_{T}$ and displacement $\boldsymbol{u}=\boldsymbol{u}_{0}$ on $\partial \Omega_{u}$. Since the tractions on the atomistic region are inherently discrete, the applied load is treated as lumped forces $f_{A}$ on individual atoms. The body contains $N$ continuum dislocations, each of which has the Burgers vector $\boldsymbol{b}^{i}$ and position $\boldsymbol{d}^{i}$, where $i$ is the number of a dislocation. The reference configuration is termed as $\boldsymbol{X}$ which is the undeformed, defect free state of the body and a displacement $\boldsymbol{u}$ brings it to the deformed configuration $\boldsymbol{x}=\boldsymbol{X}+\boldsymbol{u}$. This boundary value problem is solved for the equilibrium stress $\sigma$, strain $\epsilon$, displacement $u$ and discrete dislocation positions $\boldsymbol{d}^{i}$ in the continuum, and simultaneously for the equilibrium positions of the atoms, as a function of the imposed boundary conditions [16]. The detail description of the solution procedures have already been published in several journals [14],[68],[15],[16]. A brief idea about the atomistic and continuum 
solution procedure and dislocation detection and passing will be presented in the following sections.

\subsection{Continuum Solution Procedure}

The continuum elastic problem is solved using an approach very similar to that of the standard DD methodology described in [59]. The continuum elastic problem is divided into two complementary problems I and II, as shown in Figure 3.1. Problem I corresponds to an infinite homogeneous elastic material which contains $N$ discrete dislocations. The solution for the total stress $\tilde{\boldsymbol{\sigma}}$, strain $\tilde{\boldsymbol{\epsilon}}$ and displacements $\tilde{\boldsymbol{u}}$ in Problem I is obtained by superposition of the known elastic fields due to all the dislocations at positions $\boldsymbol{d}^{i}$ in an infinite elastic continuum, given by [16]

$$
\tilde{\boldsymbol{\sigma}}=\sum_{i}^{N} \tilde{\boldsymbol{\sigma}}^{i}, \quad \tilde{\boldsymbol{\epsilon}}=\sum_{i}^{N} \tilde{\epsilon}^{i}, \quad \tilde{\boldsymbol{u}}=\sum_{i}^{N} \tilde{\boldsymbol{u}}^{i}
$$

where, $\tilde{\boldsymbol{\sigma}}^{i}, \tilde{\boldsymbol{\epsilon}}^{i}$ and $\tilde{\boldsymbol{u}}^{i}$ are the linear elastic solutions for the fields of the $i^{\text {th }}$ dislocation in the infinite material. This superposition leads to tractions $\tilde{T}$ along the $\partial \Omega_{T}$ and some displacement $\boldsymbol{u}=\tilde{\boldsymbol{u}}$ along $\partial \Omega_{u}$ and $\partial \Omega_{I}$ which do not satisfy the overall boundary conditions $\boldsymbol{T}_{0}, \boldsymbol{u}_{I}$ and $\boldsymbol{u}_{0}$. Thus, Problem II is designed as a "corrective" boundary value problem whose fields, combined with those of Problem I, satisfy the true boundary condition. Problem II then becomes a boundary value problem for a linear elastic body, containing no dislocations in it. Because all the discontinuities and singularities are associated with the fields of Problem I, the fields of Problem II are smooth and, therefore, can be solved accurately using the finite element method.

Corrective traction $\hat{\boldsymbol{T}}=\boldsymbol{T}_{0}-\tilde{\boldsymbol{T}}$ (on $\partial \Omega_{T}$ ) and corrective displacement $\hat{\boldsymbol{u}}=\boldsymbol{u}_{0}-\tilde{\boldsymbol{u}}$ (on $\partial \Omega_{u}$ ) and $\hat{\boldsymbol{u}}=\boldsymbol{u}_{I}-\tilde{\boldsymbol{u}}$ (on $\partial \Omega_{I}$ ) are applied as boundary conditions in this case. The displacements, stresses, and strains of Problem II are denoted by $\hat{\text {. The total }}$ fields in the continuum region are then [16]

$$
\boldsymbol{u}=\tilde{\boldsymbol{u}}+\hat{\boldsymbol{u}}, \quad \boldsymbol{\sigma}=\tilde{\boldsymbol{\sigma}}+\hat{\boldsymbol{\sigma}}, \quad \boldsymbol{\epsilon}=\tilde{\boldsymbol{\epsilon}}+\hat{\boldsymbol{\epsilon}}
$$



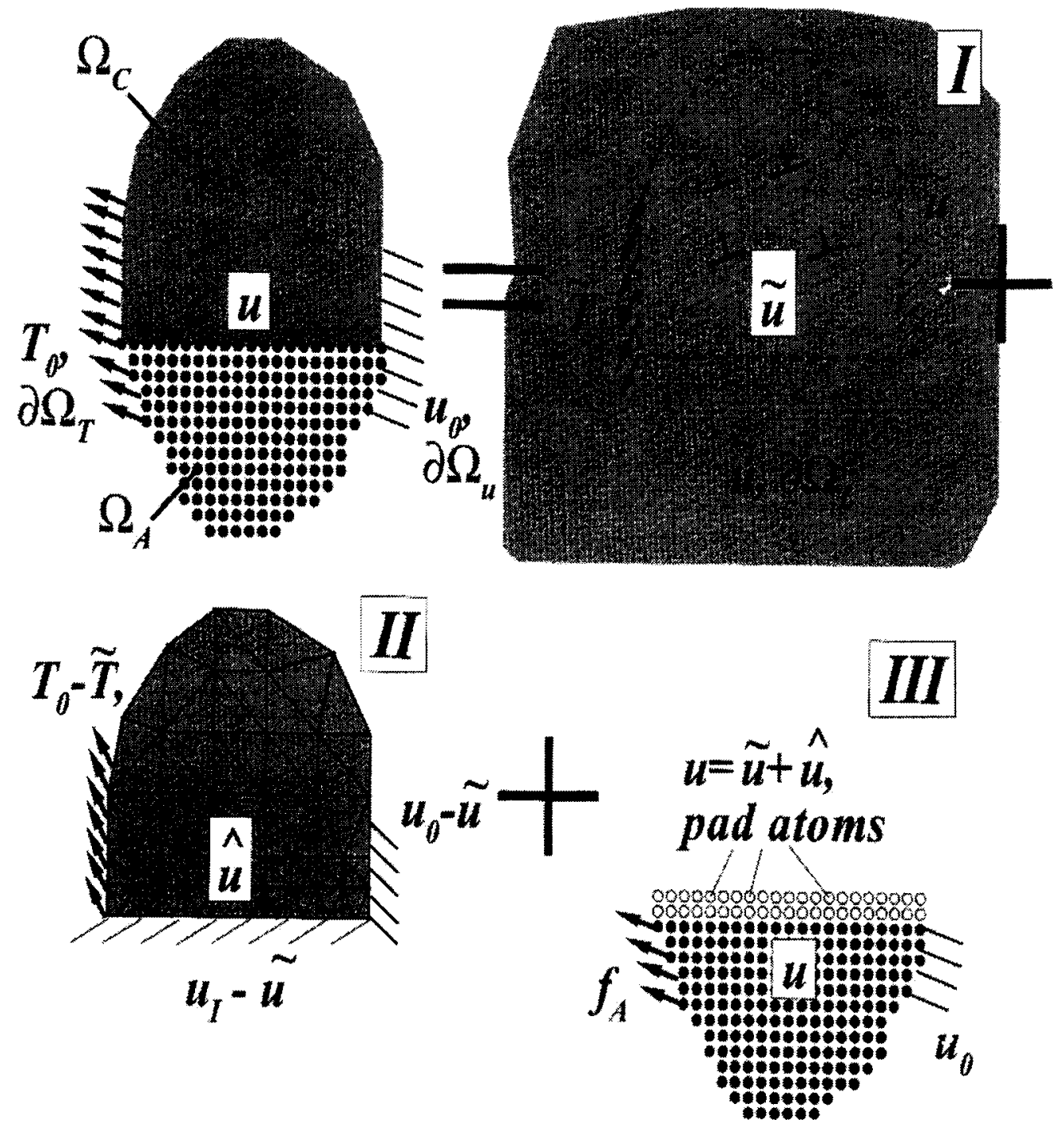

Figure 3.1: Schematic illustration of the solution procedure for the CADD method [16]. 
which satisfy the overall boundary conditions imposed on the continuum region. For Problem II, the fully anisotropic, linear elastic constitutive relation is used, so that the continuum elastic constants are matched those of the crystalline atomistic region. However, for the field of Problem I describing the discrete dislocations, isotropic elasticity with the Voigt-averaged elastic constants are used. Thus the constitutive relations in the continuum region are $[16]$

$$
\begin{gathered}
\tilde{\sigma_{i j}}=2 \mu \tilde{\epsilon_{i j}}+\lambda \tilde{\epsilon_{k k}} \delta i j \\
\hat{\sigma_{i j}}=C_{i j k l} \hat{\epsilon_{i j}}
\end{gathered}
$$

where, $\mu$ and $\lambda$ are the Voigt-average elastic constants found from the fully anisotropic modulus tensor $C_{i j k l}$. Thus, a fully anisotropic solution is obtained when there is no dislocation present in the model. But when discrete dislocations exist, small errors are introduced due to the isotropic solution of the $\sim$ field. Thus, the free energy functional $E^{C}$ for the continuum region can be written as [16]

$$
E^{C}=\frac{1}{2} \int_{\Omega_{C}}(\hat{\boldsymbol{\sigma}}+\tilde{\boldsymbol{\sigma}}):(\hat{\boldsymbol{\epsilon}}+\tilde{\boldsymbol{\epsilon}}) d V-\int_{\Omega_{T}} \boldsymbol{T}_{0}(\hat{\boldsymbol{u}}+\tilde{\boldsymbol{u}}) d A
$$

Partial derivative of Equation 3.5 with respect to the position of the dislocation $d^{i}$ yields the Peach-Koehler force $\boldsymbol{p}^{i}$ on the $i^{\text {th }}$ dislocation,

$$
p^{i}=-\left(\frac{\partial E^{C}}{\partial \boldsymbol{d}^{i}}\right)
$$

The P-K force can be obtained directly from the stress field calculated in Problems I and II. But the finite element solution to the stress field $\hat{\boldsymbol{\sigma}}$, obtained at the Gauss points of the element, is not smooth across element interfaces. Alternatively, it is also computationally feasible to calculate the P-K force numerically during the solution procedure [16]. 


\subsection{Atomistic Solution Procedure}

The atomistic region is inherently non-local, since the interaction energies of realistic interatomic potentials extend beyond-near-neighbor atoms. This region may contain any number of atomic defects such as dislocations, grain boundaries, vacancies, voids, or amorphous regions. The degrees of freedom in this region are the positions of the real atoms $\left(\boldsymbol{r}_{A}\right)$ and the interface atoms $\left(\boldsymbol{r}_{I}\right)$. The only assumption about these atomistic regions is that near the atomistic/continuum interface their behavior approaches the linear elastic response used to describe the continuum. The only nonlinear behavior allowed to "pass" across the atomistic/continuum interface is the core of a dislocation [16]. Abruptly terminating atomistic regions, or abruptly beginning a finite element description of the material, introduces a spurious surface energy and non-physical interface reconstructions, as the atoms along the interface may not have proper atomic coordination for the interatomic interactions. To overcome this problem and to ensure proper coordination of the atoms at and near the interface, a number of pad atoms has been introduced that overlaps with the continuum region. The pad region is shown in Figure 3.2. The pad thickness must be such that real atoms can see a full complement of their neighbor atoms during the solution process and the minimum thickness of the pad must exceed the range of interatomic interactions $r_{c u t}$ in the material. As the pad atoms reside on the continuum region, their positions are determined by interpolating the nodal displacements obtained from the continuum solution. From the viewpoint of the real atoms and interface atoms, the pad atom positions are instantaneously fixed. Denoting as $E_{i}$ the energy of the $i^{t h}$ atom as determined from the EAM function, the total atomistic free energy functional is given by [16]

$$
E^{a}=\sum_{i \in(A, I, P)} E_{i}\left(\boldsymbol{r}_{A}, \boldsymbol{r}_{I}, \boldsymbol{r}_{P}\right)-\boldsymbol{f}_{A} \cdot \boldsymbol{u}_{A}
$$


where, $\boldsymbol{u}_{A}$ are the atom displacements. The sum over atoms includes real atoms $(A)$, interface atoms $(I)$ and pad atoms $(P)$. Partial derivatives of $E^{a}$ with respect to the positions of real atoms $\left(\boldsymbol{r}_{A}\right)$ and interface atoms $\left(\boldsymbol{r}_{I}\right)$ then yield full atomistic force on the atoms $[16]$,

$$
\boldsymbol{f}=-\left(\frac{\partial E^{a}}{\partial \boldsymbol{r}_{A I}}\right)
$$

The total free energy for the atomistic domain includes interactions with the pad atoms, but partial derivatives of $E^{a}$ with respect to the pad atoms would generally induce non-physical surface effects. Thus, these derivatives are simply omitted because they are not needed [16]. Other than ensuring proper coordination of the real atoms in the vicinity of the interface, the pad atoms also inform the atomistic region about the deformation in the continuum region. Similarly, the interface atoms inform the continuum about the deformation in the atomistic region. Due to the non-local nature of the atoms, a finite thickness pad is required to transmit the continuum deformation to the atoms. In contrast, because the finite element continuum is local, only a set of interfacial atoms are required to transmit the atomistic deformation to the continuum region.

\subsection{Equilibrium configuration}

After each increment of external loading it is necessary to bring the entire system to the equilibrium. Unlike other coupling scheme (i.e. QC and CLS) CADD does not have a well-defined energy functional for the entire system. Thus, to drive the entire system toward equilibrium after each load step the standard, computationally efficient, conjugate gradient method can not be used. Alternatively, the CADD method adopts a modified conjugate gradient minimization scheme, named Pseudo Conjugate Gradient approach, to search for the point of zero forces on all degrees of freedom rather than minimizing the total energy. The force values are obtained from 
the derivatives of the atomistic and continuum energy functional given in Equation 3.7 and 3.5 respectively. At the beginning of each iteration the direction of descent is chosen using the standard conjugate gradient procedure and along this direction, a point of zero forces is determined. At such a point, the vector of forces is perpendicular to the direction of descent and is used to choose the direction for the next iteration. The atomic coordinates and dislocation positions are updated during each search step of the CG minimization. Using this revised atom and dislocation positions, the continuum displacements and the positions of pad atoms are updated $[16],[14]$.

\subsection{Dislocation Detection and Passing}

\subsubsection{Dislocation Detection}

The key strength of the CADD approach and the feature that makes it unique and powerful is its ability to detect dislocations that have been nucleated in the atomistic region and that are moving towards the continuum region during the solution procedure. This is done by defining a "detection band" inside the atomistic region at a short distance from the atomistic/continuum interface, as shown in Figure 3.2. The detection band consists of small triangular elements, with each element sitting on three different slip planes. If a dislocation passes along one of these planes, it generates a Lagrangian finite strain in the element of [68]

$$
E_{i j}=\frac{\left(b_{i} m_{j}+m_{i} b_{j}\right)}{2 d}+\frac{m_{i} b_{k} b_{k} m_{j}}{2 d^{2}}
$$

where, $\boldsymbol{m}$ is the slip plane normal, $\boldsymbol{b}$ is the burgers vector, $d$ is the interplanar spacing. For a given crystal structure and orientation, all the possible slip systems and associated strains $\boldsymbol{E}$ are computed by a subroutine. The code is equipped for FCC, Hexagonal and BCC crystals. The detection algorithm monitors the strains in the detection band elements and compares them to all possible dislocation slip 


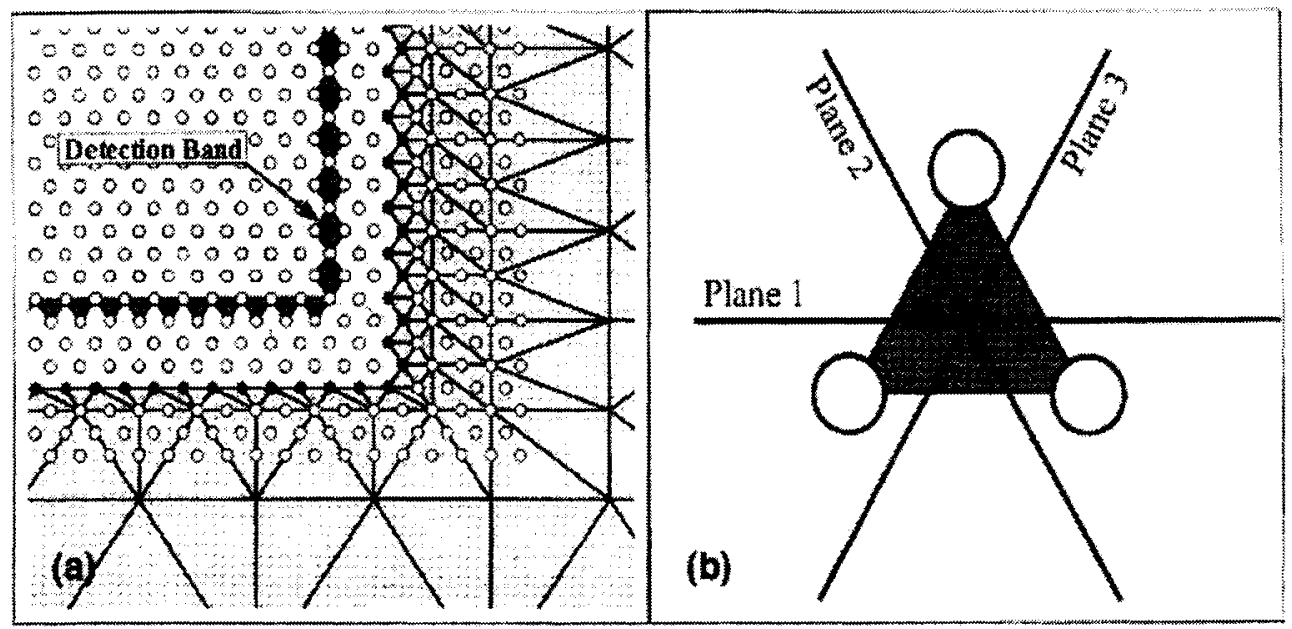

Figure 3.2: (a) Dark grey elements are used in dislocation detection. Filled circles are interface atoms and the open circles are the pad atoms that reside on the finite elements (b) A close up of one detection element, indicating the three slip planes on which it lies [16].

strains after each CG energy minimization step. When the strain in an element corresponds to a particular dislocation, the dislocation core is assumed to reside at the centroid of that element and the slip plane and burgers vector can also be identified [16],[68]. During the simulation many dislocations may pass through a single detection band element. Thus, it is necessary to filter out the contributions from all previous slip activities and consider only the displacements resulting from new defects [68]. Moreover, the elastic deformation, rigid body translation and rotation associated with the detection band element must also be eliminated [16].

\subsubsection{Dislocation Passing}

After being detected, the dislocation is passed to the continuum as a discrete dislocation. This is done by artificially shifting the core of the dislocation along its slip plane from its location in the detection band to a location across the interface in the continuum region. At the same time, the continuum elastic displacement of a dislocation dipole is superimposed onto those of the atoms and nodes. These 


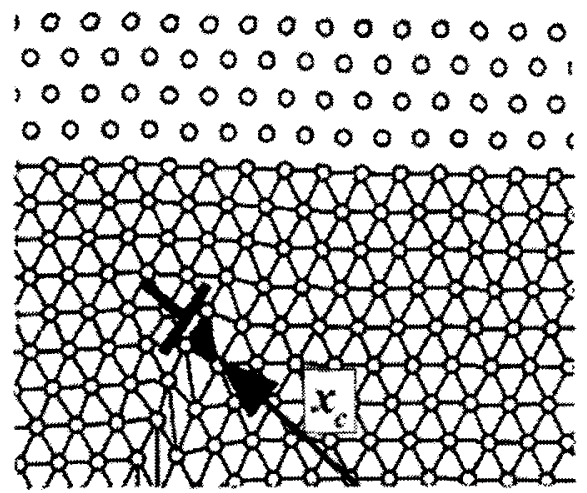

(a)

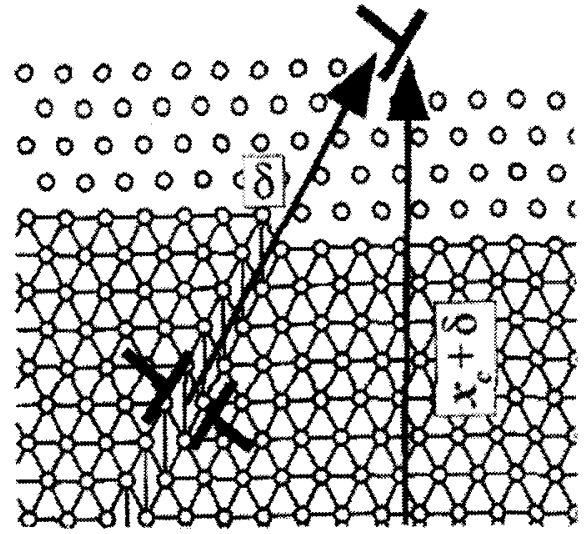

(b)

Figure 3.3: (a) The dislocation is detected at position $\mathbf{x}_{c}$ (b) dislocation is passed across the atomistic/continuum interface at position $\mathbf{x}_{c}+\boldsymbol{\delta}$ [16].

displacements cancel the original core in the atomistic region at $\boldsymbol{x}_{c}$ and replaces it with a continuum dislocation at the position $\boldsymbol{x}_{c}+\boldsymbol{\delta}$ in the continuum region, shown in Figure 3.3. Once this core is in the continuum region, it is added to the array of discrete dislocations [16],[68]. While passing the dislocation from the continuum region back to the atomistic region, it is artificially pushed into the atomistic region by superposition of its linear elastic displacement fields on the atom positions. In this case the passing is done only when the dislocation comes to within a small tolerance of the interface. The core of the introduced dislocation is quickly relaxed to the correct atomistic core in the subsequent energy minimization steps [16]. The total solution procedure of the CADD method is illustrated in Figure 3.4.

\subsection{Modification of the CADD model}

The CADD method had previously been applied to examine the phenomena of 2D nano-indentation into a single crystal Aluminum film bonded to a rigid substrate [14]. Both thick and thin hexagonal aluminum films were studied during 


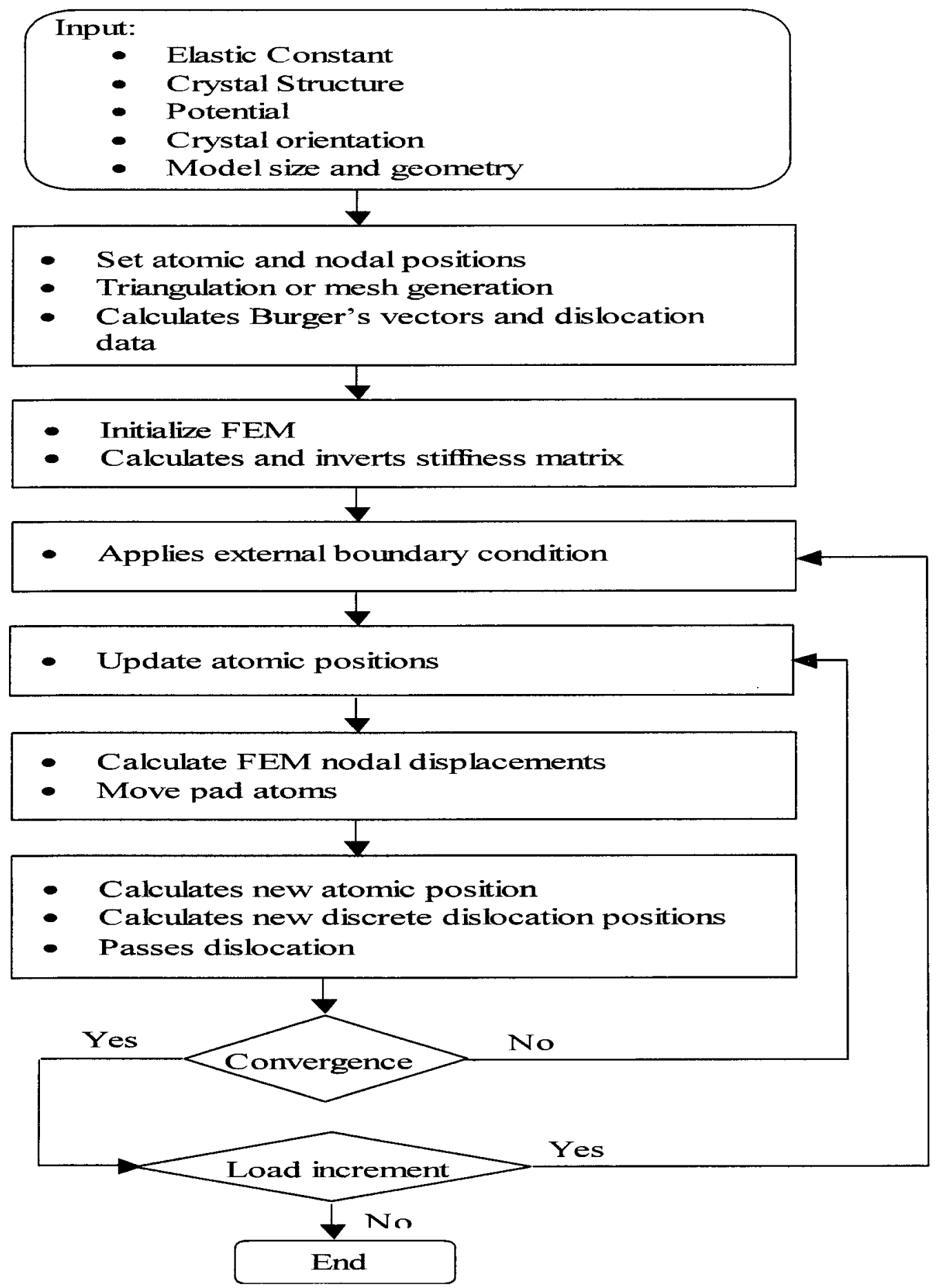

Figure 3.4: CADD flow chart. 
displacement-controlled and force-controlled nano-indentation experiments. The present research was started with an aim to modify the existing single grain CADD model for multiple grains. One of our main objectives was to use this code to investigate the phenomena associated with nano-indentation into a layered structure. Two materials, i.e. Nickel and Aluminum, having distinct material properties were selected for this present work. Nickel is characterized by its higher hardness than aluminum, which enabled us to study a bilayered structure consists of a soft film coated on a hard substrate and vice versa. A multilayer structure having alternating layers of hard and soft materials can also be studied. The significance of such a multilayer structure has already been discussed in Chapter 1. Moreover, the effects of lattice constant mismatch and elastic constant mismatch between the two adjacent layers on dislocation nucleation, dislocation movement and dislocation-interface interaction can also be revealed.

\subsubsection{Selection of the potential}

To investigate the structures and the thermodynamic properties of a system and to obtain meaningful results from atomistic simulations of materials, the selection of accurate potentials is of considerable interest. The usefulness of the results strongly depends upon the quality of the interatomic potential employed in the simulation. For metallic system, the traditional approach of using pairwise potentials have known deficiencies to investigate the defective regions such as crack tips and free surfaces. Daw and Baskes's idea of adding the embedded energy term for every atom to the pairwise potential have shown dramatic improvement in simulation results over pair potentials without severe computational effort. Using a similar approach, Voter and Chen [69] fit an interatomic potential to the $\mathrm{Ni}_{3} \mathrm{Al}$ alloy system. This potential can treat fcc $\mathrm{Ni}$, fcc $\mathrm{Al}$ and the $\mathrm{Ni}_{3} \mathrm{Al}$ alloy [69]. Details of the fitting procedure are presented in [69]. The potential gives a perfect fit to the experimental values of 
lattice constant $\left(a_{o}\right)$, cohesive energy $\left(E_{c o h}\right)$ and bulk modulus (B) for any choice of pair potential $(\Phi(r))$ and electron density $(\rho(r))$. Other material properties i.e. the elastic constants, the vacancy formation energy, the bond length and the bond energy of the diatomic molecule also show good agreement with experimental results [69]. Figure 3.5 shows all the functions for the $\mathrm{Ni}, \mathrm{Al}$ and $\mathrm{Ni}_{3} \mathrm{Al}$ potentials. This figure demonstrates that the repulsive nature of the embedding function is balanced by the attractive pairwise potential and for the pure metals, the total attraction of $\mathrm{Ni}$ for $\mathrm{Al}$ is actually stronger than that of $\mathrm{Ni}$ for $\mathrm{Ni}$ or $\mathrm{Al}$ for $\mathrm{Al}$ [69]. Table 3.1 and Table 3.2 show the experimental data used in the fits along with the calculated values of Voter-Chen potential for Aluminum and Nickel respectively.
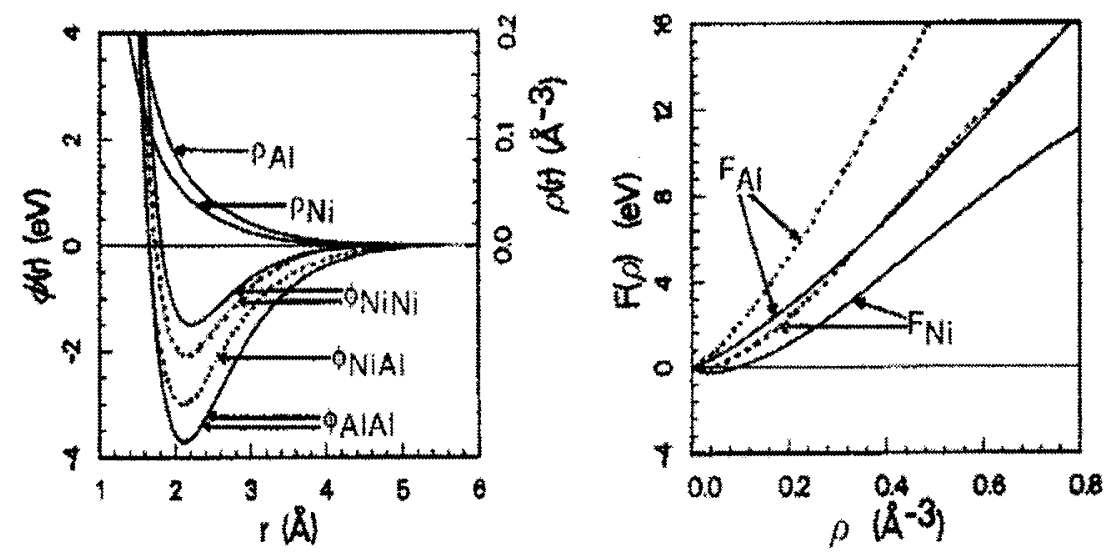

Figure 3.5: The functions comprising the $\mathrm{Ni}, \mathrm{Al}$ and $\mathrm{Ni}_{3} \mathrm{Al}$ potentials. The dotted lines represent the variation of the functions with respect to scaling of electron density $(\rho)$ [69].

\section{Indenter potential}

A rigid, frictionless, cylindrical indenter is chosen for indentation which is defined by a repulsive potential of the form

$$
\Phi= \begin{cases}C(r-R)^{2} & \text { for } r<R \\ 0 & \text { for } r \geq R\end{cases}
$$




\begin{tabular}{|c|c|c|}
\hline Property of aluminum & expt. & calc. \\
\hline$a_{o}(\AA)$ & 4.05 & 4.05 \\
\hline$E_{c o h}(\mathrm{eV})$ & 3.36 & 3.36 \\
\hline$B\left(10^{12} \mathrm{erg} / \mathrm{cm}^{3}\right)$ & 0.79 & 0.79 \\
\hline$C_{11}\left(10^{12} \mathrm{erg} / \mathrm{cm}^{3}\right)$ & 1.14 & 1.07 \\
\hline$C_{12}\left(10^{12} \mathrm{erg} / \mathrm{cm}^{3}\right)$ & 0.619 & 0.652 \\
\hline$C_{44}\left(10^{12} \mathrm{erg} / \mathrm{cm}^{3}\right)$ & 0.316 & 0.322 \\
\hline Vacancy formation energy $\Delta E_{1 V}^{f}(\mathrm{eV})$ & 0.75 & 0.73 \\
\hline Bond Energy $D_{e}(\mathrm{eV})$ & 1.60 & 1.54 \\
\hline Bond length $R_{e}(\AA)$ & 2.47 & 2.45 \\
\hline
\end{tabular}

Table 3.1: Experimental and calculated properties of aluminum [69].

\begin{tabular}{|c|c|c|}
\hline Property of nickel & expt. & calc. \\
\hline$a_{o}(\AA)$ & 3.52 & 3.52 \\
\hline$E_{\text {coh }}(\mathrm{eV})$ & 4.45 & 4.45 \\
\hline$B\left(10^{12} \mathrm{erg} / \mathrm{cm}^{3}\right)$ & 1.81 & 1.81 \\
\hline$C_{11}\left(10^{12} \mathrm{erg} / \mathrm{cm}^{3}\right)$ & 2.47 & 2.44 \\
\hline$C_{12}\left(10^{12} \mathrm{erg} / \mathrm{cm}^{3}\right)$ & 1.47 & 1.49 \\
\hline$C_{44}\left(10^{12} \mathrm{erg} / \mathrm{cm}^{3}\right)$ & 1.25 & 1.26 \\
\hline Vacancy formation energy $\Delta E_{1 V}^{f}(\mathrm{eV})$ & 1.60 & 1.60 \\
\hline Bond Energy $D_{e}(\mathrm{eV})$ & 1.95 & 1.94 \\
\hline Bond length $R_{e}(\AA)$ & 2.20 & 2.23 \\
\hline
\end{tabular}

Table 3.2: Experimental and calculated properties of nickel [69]

where, $C=100 \mathrm{eV} / \AA^{2}$ is chosen empirically, $r$ is the distance between atom center and indenter center and $R=50 \AA$ is chosen in this study as the indenter radius. The $\mathrm{Al}$ and $\mathrm{Ni}$ atoms interact with this potential, which effectively repels the atoms and forces them to remain outside the $50 \AA$ radius of the indenter.

\subsubsection{Lattice constant calculation for $\mathrm{Al}$ and $\mathrm{Ni}$}

For our research we selected 2D hexagonal structures of Aluminum and Nickel with their $\mathrm{z}$-axis perpendicular to the in-plane deformation. The hexagonal structure is not a "real" crystal structure, and it is unstable both in 3D and out-of-plane defor- 
mation. It is stable in $2 \mathrm{D}$ because it is constrained in the $\mathrm{z}$ direction which allows us to get 2D in-plane deformation. Another feature of such 2D hexagonal crystal structure is that, a dislocation gliding on one of the three slip systems can dissociate into two dislocations, one on each of the other two systems. Such dissociation can be viewed as a $2 \mathrm{D}$ analogue to cross-slip in $3 \mathrm{D}[16]$. Also, the dislocation character can only be edge with its line direction perpendicular to the $\mathrm{x}-\mathrm{y}$ plane of the model, simplifying the details of dislocation detection and passing. Morcover, the elastic properties are exactly isotropic in-plane for hexagonal materials. These features of hexagonal crystal structure motivate us in using such fictitious model material for our simulation. Our simulations cannot be used, therefore, to make accurate quantitative predictions about real materials. Instead, they permit us to examine trends and generic behaviors that real materials may also exhibit.

The Voter-Chen EAM potential [69] was used to create the structures of these two materials. Initially the positions of a number of atoms for each material were generated using a separate computer program. Then the structures were allowed to relax using the molecular dynamics code (Dynamo [70]) that finds the equilibrium configuration of the atoms using the Voter-Chen potential. The equilibrium condition to calculate the lattice constants is given by [57]

$$
A_{i j}+F^{\prime}(\bar{\rho}) V_{i j}=0
$$

where

$$
\begin{aligned}
A_{i j} & =\frac{1}{2} \sum_{m} \Phi_{m}^{\prime} a_{i}^{m} a_{j}^{m} / a^{m} \\
V_{i j} & =\frac{1}{2} \sum_{m} \rho_{m}^{\prime} a_{i}^{m} a_{j}^{m} / a^{m}
\end{aligned}
$$

and where $a_{i}^{m}$ is the $i^{t h}$ component of the position vector to the $m^{t h}$ neighbor, $\Phi_{m}^{\prime}=[d \Phi(r) / d r]_{r=a^{m}}$ and $\rho_{m}^{\prime}=[d \rho(r) / d r]_{r=a^{m}}$, (see section 2.6.1 for more detail). From the relaxed configuration of the atoms, the Bravais lattice vectors of each 
material were calculated. Figure 3.6 shows the structures of these two materials after relaxation. For $\mathrm{Al}$, the Bravais vectors are:

$$
\begin{array}{r}
b_{1}=[2.7663137,0.0,0.0] \\
b_{2}=[1.383156,2.395698,0.0] \\
b_{3}=[0.0,0.0,3.0]
\end{array}
$$

and for $\mathrm{Ni}$, the Bravais vectors are:

$$
\begin{array}{r}
b_{1}=[2.409421,0.0,0.0] \\
b_{2}=[1.204710,2.086620,0.0] \\
b_{3}=[0.0,0.0,3.0]
\end{array}
$$

Although, as we said earlier, the hexagonal structure is $2 \mathrm{D}$ and is constrained in the z-direction, it is necessary to specify the equilibrium spacing between layers (for hexagonal material, this is only one layer) in the z-direction to generate an infinite crystal using DYNAMO [70]. For the DYNAMO code to work, this spacing should be greater than half of the cutoff radius of the EAM potential. We chose the spacing $3.0 \AA$ in the $\mathrm{z}$-direction for both $\mathrm{Al}$ and $\mathrm{Ni}$, as the cutoff radius for the Voter-Chen EAM potential is $5.5 \AA$. Although $\mathrm{Al}$ and $\mathrm{Ni}$ may have different independent values, we were able to select only one periodic length in the z-direction. Thus, we had to stretch one or compress the other along the z-direction to make them fit together.

\subsubsection{Elastic modulus Calculation}

In the CADD formulation, a fully anisotropic and linear elastic constitutive relation is used for the fields of Problem II, that describes the finite element solution of the total continuum field, to ensure a match of elastic constants in the continuum region to those of the crystalline atomistic region. However, for the fields of Problem I describing the discrete dislocation, we use the analytic fields from isotropic elasticity 


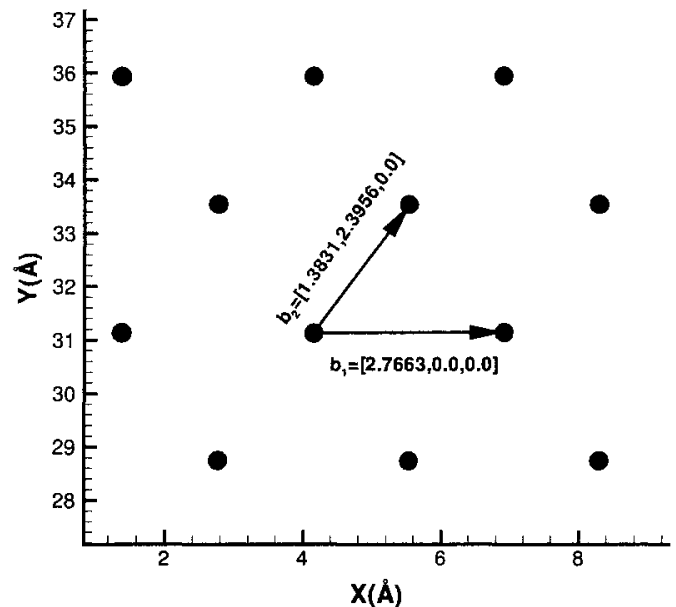

(a)

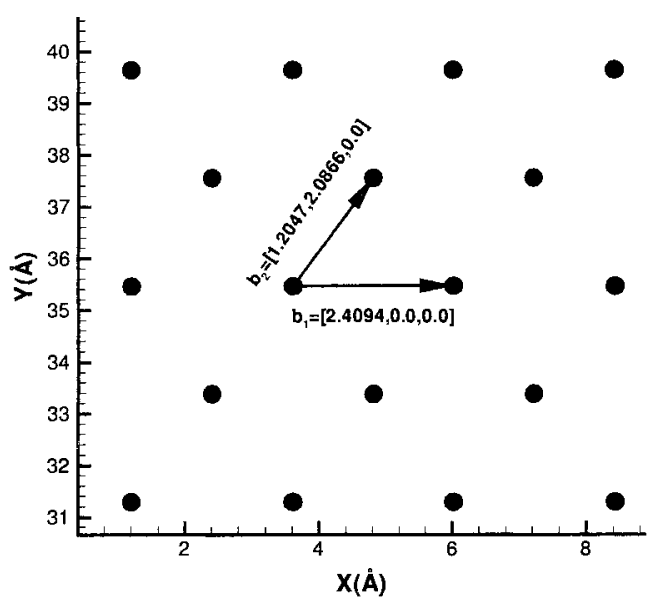

(b)

Figure 3.6: Bravais lattice vectors of (a) Aluminum and (b) Nickel.

with Voigt-averaged elastic constants. The constitutive relations in the continuum region are shown in Equation 3.3 and 3.4. The macroscopic elastic moduli $C_{i j k l}$ in Equation 3.4 can be written in terms of the microscopic interatomic interactions. At equilibrium, these moduli take the form [57]

$$
C_{i j k l}=\left(B_{i j k l}+F^{\prime}(\bar{\rho}) W_{i j k l}+F^{\prime \prime}(\bar{\rho}) V_{i j} V_{k l}\right) / \Omega_{0}
$$

where $\Omega_{0}$ is the undeformed atomic volume, and

$$
\begin{aligned}
B_{i j k l} & =\frac{1}{2} \sum_{m}\left(\Phi_{m}^{\prime \prime}-\Phi_{m}^{\prime} / a^{m}\right) a_{i}^{m} a_{j}^{m} a_{k}^{m} a_{l}^{m} /\left(a^{m}\right)^{2} \\
W_{i j k l} & =\sum_{m}\left(\rho_{m}^{\prime \prime}-\rho_{m}^{\prime} / a^{m}\right) a_{i}^{m} a_{j}^{m} a_{k}^{m} a_{l}^{m} /\left(a^{m}\right)^{2} \\
V_{i j} & =\sum_{m} \rho^{\prime} a^{m} a_{i}^{m} a_{j}^{m} / a^{m} .
\end{aligned}
$$

where, $\Phi_{m}^{\prime \prime}=\left[d^{2} \Phi(r) / d r^{2}\right]_{r=a^{m}}$ and $\rho_{m}^{\prime \prime}=\left[d^{2} \rho(r) / d r^{2}\right]_{r=a^{m}}$

This expression for the elastic modulus demonstrates an important connection between atomistic and continuum models and are used for the finite element problem. 
This expression will yield three independent non-zero components of the moduli for a crystal with cubic symmetry. In Voigt notation they are expressed as follows [57]

$$
\begin{array}{r}
C_{1111}=C_{11}=\left[B_{11}+F^{\prime}(\bar{\rho}) W_{11}+F^{\prime \prime}(\bar{\rho})\left(V_{11}\right)^{2}\right] / \Omega_{0}, \\
C_{1122}=C_{12}=\left[B_{12}+F^{\prime}(\bar{\rho}) W_{12}+F^{\prime \prime}(\bar{\rho})\left(V_{11}\right)^{2}\right] / \Omega_{0}, \\
C_{1212}=C_{44}=\left[B_{12}+F^{\prime}(\bar{\rho}) W_{12}\right] / \Omega_{0},
\end{array}
$$

The materials studied in this work are hexagonal $\mathrm{Al}$ and $\mathrm{Ni}$ oriented with the z-axis out-of-plane, so as to create an effectively $2 \mathrm{D}$ atomistic region. Using the Voter-Chen EAM potential, the calculated in-plane elastic constants of aluminum and nickel are presented in Table 3.3 and Table 3.4 respectively.

\begin{tabular}{|c|c|}
\hline$C_{11}=C_{22}$ & $1.5126 \mathrm{eV} / \AA^{3}$ \\
\hline$C_{12}=\lambda$ & $0.9485 \mathrm{eV} / \AA^{3}$ \\
\hline$C_{66}=\mu$ & $0.28203 \mathrm{eV} / \AA^{3}$ \\
\hline
\end{tabular}

Table 3.3: Calculated in-plane elastic constants for aluminum.

\begin{tabular}{|c|c|}
\hline$C_{11}=C_{22}$ & $4.8722 \mathrm{eV} / \check{\AA}^{3}$ \\
\hline$C_{12}=\lambda$ & $2.4986 \mathrm{eV} / \AA^{3}$ \\
\hline$C_{66}=\mu$ & $1.1868 \mathrm{eV} / \AA^{3}$ \\
\hline
\end{tabular}

Table 3.4: Calculated in-plane elastic constants for nickel.

\subsubsection{Model description for bilayer simulation}

Figure 3.7 shows a bilayer simulation model, in which a thin film of one material was placed on top of a substrate of other material. Different film thicknesses (i.e. $40 \AA$ or $50 \AA$ ) were chosen for different bilayer simulation models but the overall size of the model was kept constant at $920 \AA$ by $530 \AA$. The radius of the indenter was $50 \AA$. We defined one atomistic region beneath the indenter for both of these two layers. 
The thickness and the width of the atomistic region were chosen to be $160 \AA$ and $110 \AA$ respectively. To study the nucleation of the dislocations beneath the indenter, and the interactions between the dislocations with the interface and to study the role of misfit dislocations in the deformation process, a significant portion of the whole system should be considered as an atomistic region under the indenter. Thus, the width of the atomistic region is selected to be greater than the diameter of the indenter and the thickness is chosen to be greater than the film thickness. Figure 3.8 shows the atomistic region of a $50 \AA$ nickel film on top of an aluminum substrate model. The atoms are at the vertices of the small triangular elements, which are shown only to make visualization of the deformation easier. We can also see the interface between these two layers in the middle. Although we defined one atomistic region for both of these two layers, the atom spacings are not uniform in the whole region. Above the layer interface, the atoms are placed into their Bravais lattice sites based on the lattice constant of material 1 i.e., nickel. But below the layer interface, a new lattice constant of material 2 i.e., aluminum, is used to determine the positions of the atoms. On the other hand, we defined several continuum regions around the atomistic region. In these continuum regions, the size of the finite elements are set to increase gradually as they move away from the atomistic/continuum interface, Figure 3.9. But still the nodes of the elements represent the Bravais lattice sites of the crystal. The last row of atoms in the atomistic region is considered as the interface nodes between atomistic region and continuum region. After defining the positions of the real atoms and the continuum nodes, the pad atoms are added to the model. The pad atoms occupy the physical space of the finite elements and their positions are determined in a similar way as for the real atoms, Figure 3.9. The thickness of the pad region should be equal to or greater than the cut of radius of the interatomic potentials. For the Voter-Chen interatomic potential it is $5.5 \AA$. After creating the model, it becomes necessary to specify the materials of each atom 


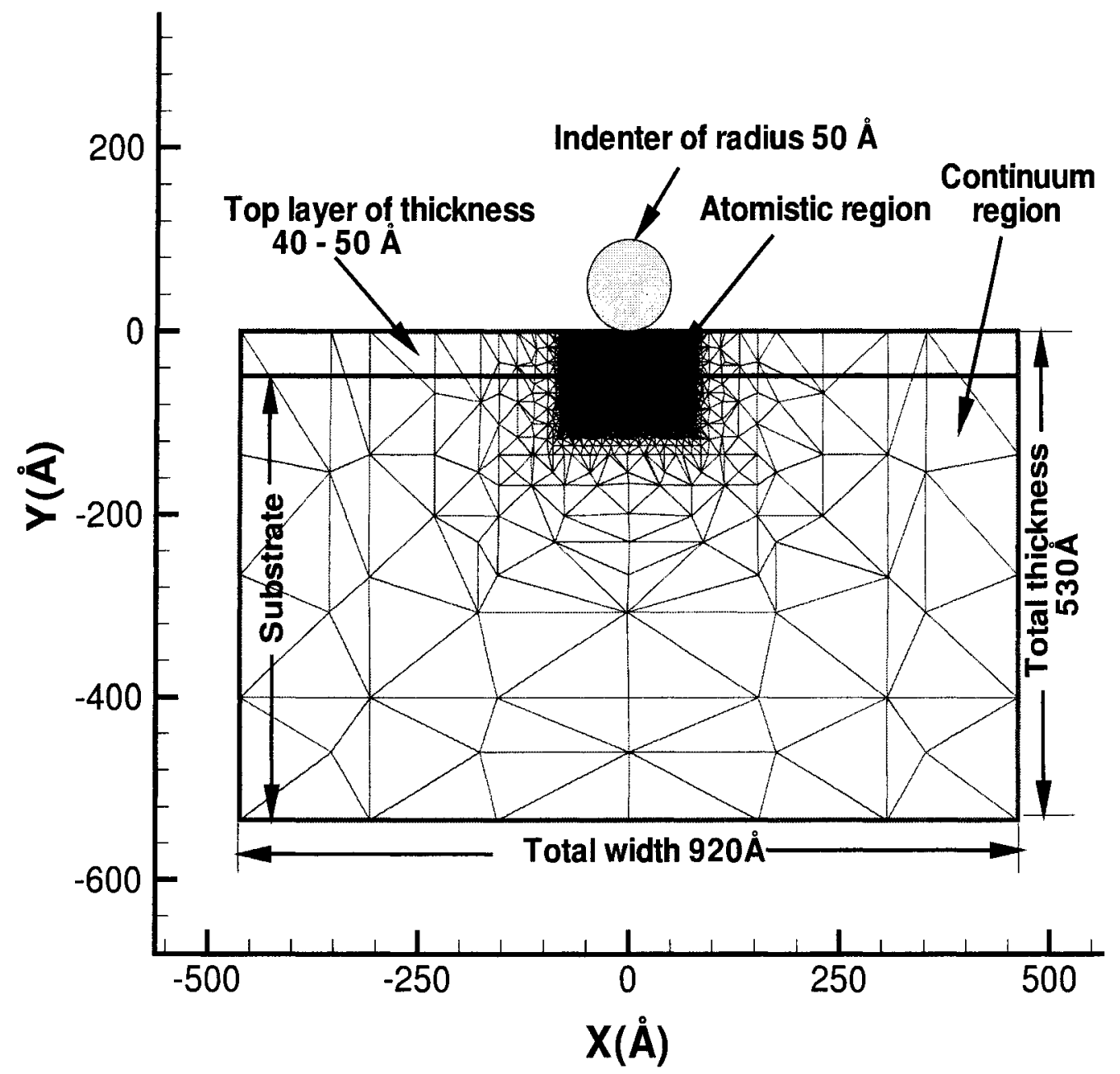

Figure 3.7: Bilayer simulation model. 


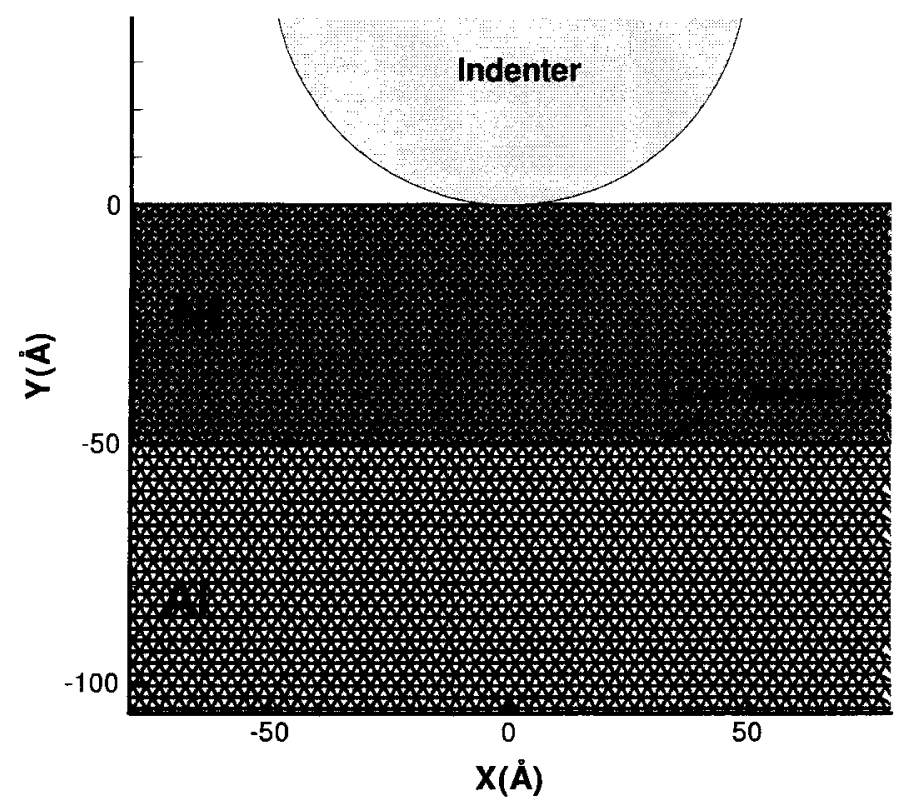

Figure 3.8: Atomistic region under the indenter and the position of the layer interface between $\mathrm{Ni}$ and $\mathrm{Al}$.

and the continuum element. In the atomistic region it can be done in a straight forward way. Any atom located above the interface are considered as material 1, and rest of the atoms below the interface are considered as material 2. But the materials of the continuum elements are defined in a slightly different way. If the centroid of an element falls above the interface then the material of that element is considered as material 1, otherwise, elements having their centroid below the interface are considered as material 2. Although we specified a uniform thickness of the film at the beginning, we obtained a nonuniform interface in the continuum region as sizes of the elements increase gradually as we move further from the interface, Figure 3.10. We assume that, this discrepancy will not introduce significant error in our results, since it is at a very small length scale. 


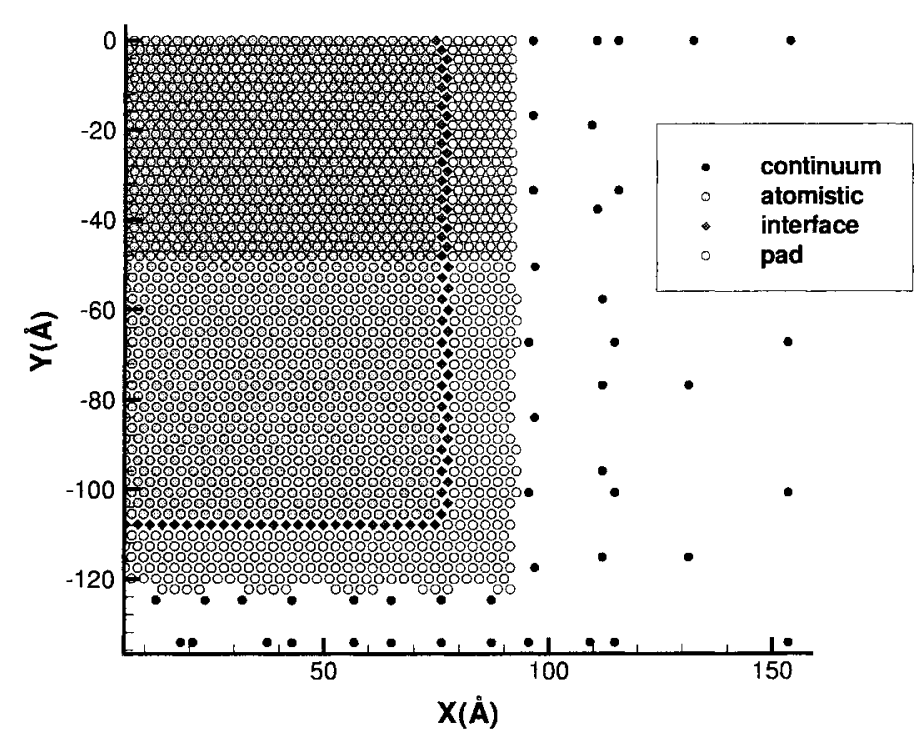

Figure 3.9: Distribution of real atoms (light gray circles), interface atoms (dark gray diamonds), pad atoms (white circles) and continuum nodes (black circles)in bilayer simulation model.

\subsubsection{Model description for multilayer simulation}

Figure 3.11 shows the geometry of a multilayer simulation model, in which, one layer of $\mathrm{Ni}$ and another layer of $\mathrm{Al}$ films are placed on top of a Ni substrate. The thickness of the films and the substrate are shown in the figure. The size of the models for every multilayer simulation case were kept fixed at $920 \AA$ by $600 \AA$. The figure also shows the positions of the two interfaces of this model. The key feature of this model is the addition of a second atomistic region near the second interface. An important point to be noted here is the size of this region, which is little bit wider than that of the first atomistic region. It is necessary to make it wider as the dislocations nucleated in the top layers, spread out as they come down to the bottom layers. We also made it very thin and considered only several rows of atoms on the either side of the interface, to study the interactions between the dislocations with the interface 


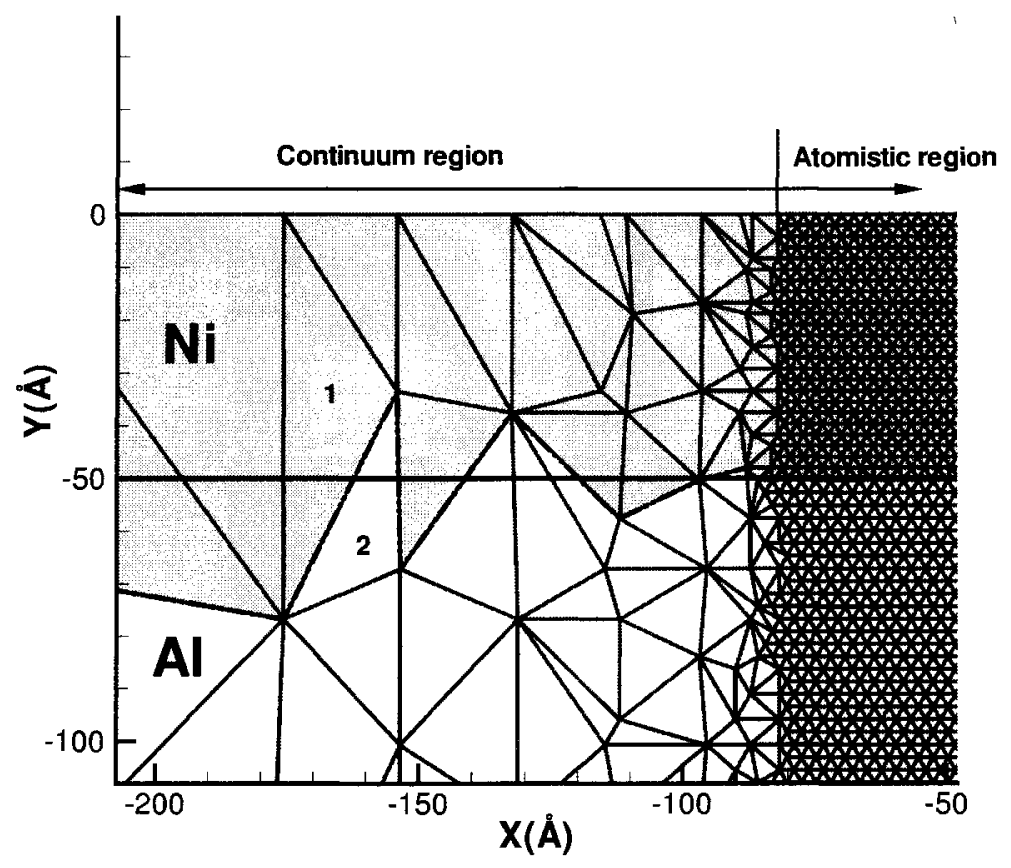

Figure 3.10: Actual position of the interface in the continuum region (marked by dashed line), whereas, solid line defines the defined film thickness.

and with the misfit dislocations but without increasing the computational effort significantly. It is possible to reduce the system size further by considering several small atomistic regions along the interface, in the positions where dislocations may interact with the interface, but that will increase the complexity of the modelling. Figure 3.12 shows a close view of the positions of the real atoms, interface atoms, pad atoms and the continuum nodes around the second interface. Here we see a second layer of $\mathrm{Al}$ film (more widely spaced atoms above the interface) is placed on top of Ni substrate. These positions have been determined in the same way as we did for the bilayer models. The pad atoms are also added around the atomistic region based on the lattice constants of these two materials. As we introduced an additional atomistic region, a second detection band is needed to be defined inside the second atomistic region to detect the dislocations coming down from the top layers. Figure 3.13 shows the position of this second detection band inside the atomistic region. 


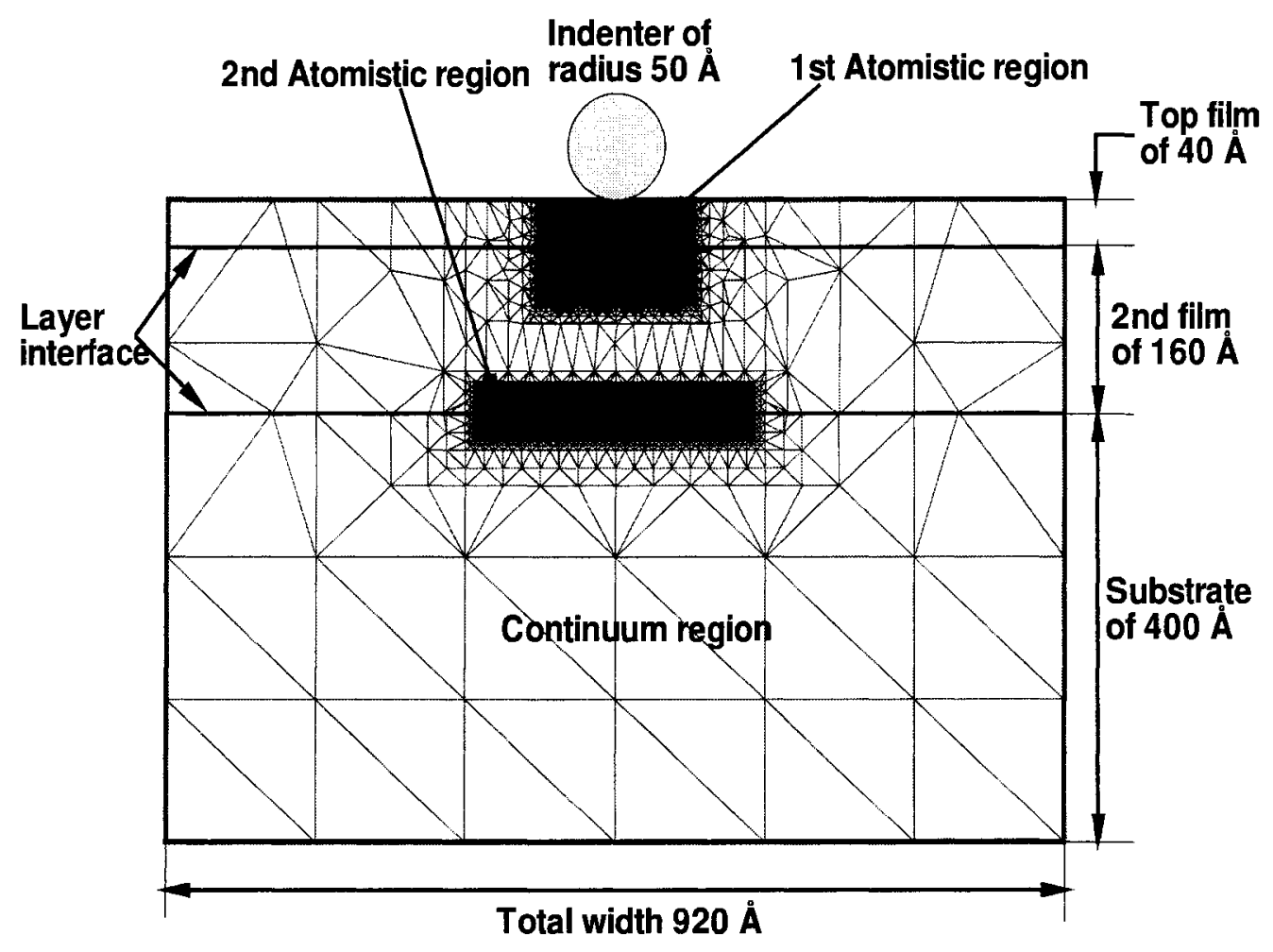

Figure 3.11: Multilayer simulation model.

Thereafter, a slightly different multilayer simulation model has been studied, in which, we considered an identical multilayer simulation model as before, but ignored the second atomistic region near the second interface. This was done intentionally by replacing the atoms at that region with the continuum nodes. Thus, the gray colored atoms in Figure 3.12 became the continuum nodes and the white colored elements in Figure 3.13, which were initially the mesh joining those atoms, became finite elements having different elastic constants across the interface. In brief, there was a fully atomistic mismatch across the interface before, but only the elastic constant mismatch after doing the modification. All other parameters, i.e. total number of 


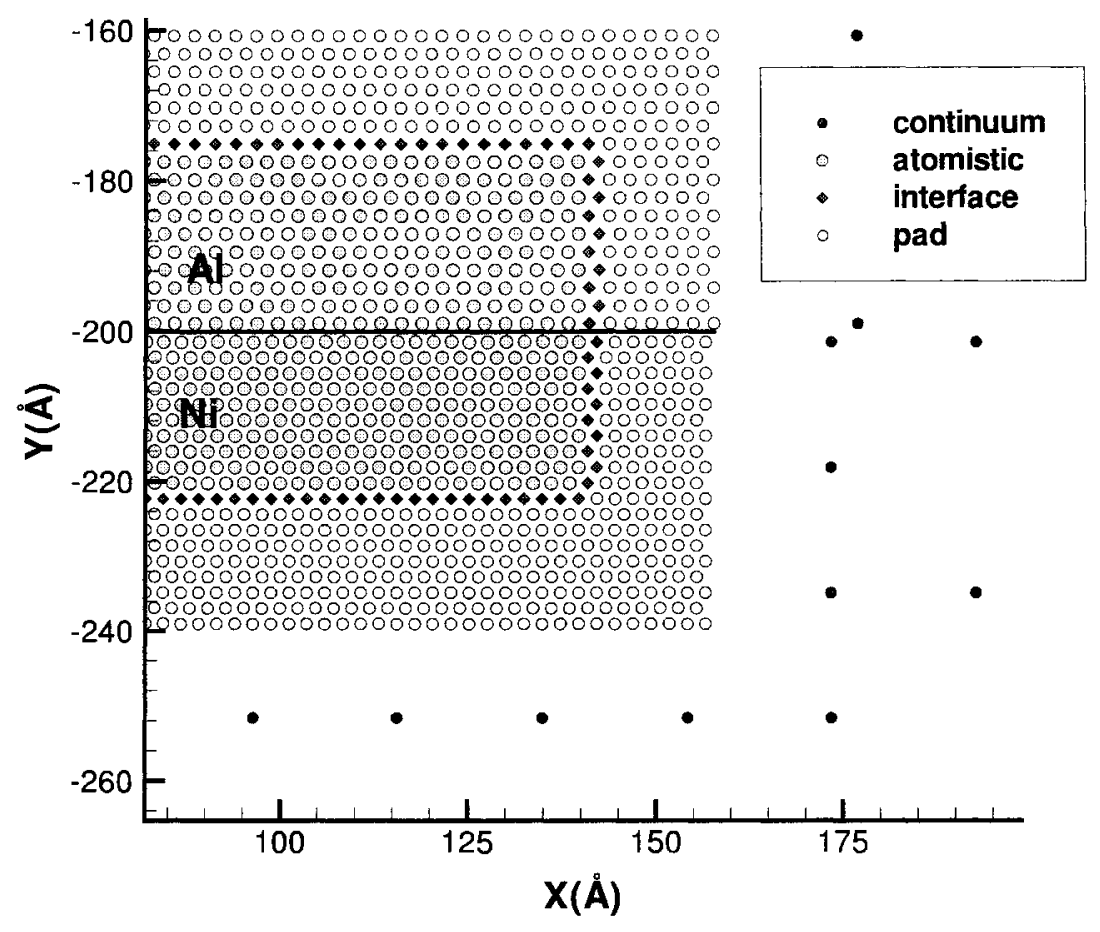

Figure 3.12: Positions of real atoms (light gray circles), interface atoms (dark gray diamonds), pad atoms (white circles) and continuum nodes (black circles) around the second atomistic region.

the pad atoms, layer thicknesses etc., were kept constant. Our objective was to study the change in behavior of the systems if we neglect the atomistic details in the regions where those are important. Moreover, that also allowed us to see the effectiveness of the continuum model to predict the dislocation behavior near the interface compared to the atomistic model. 


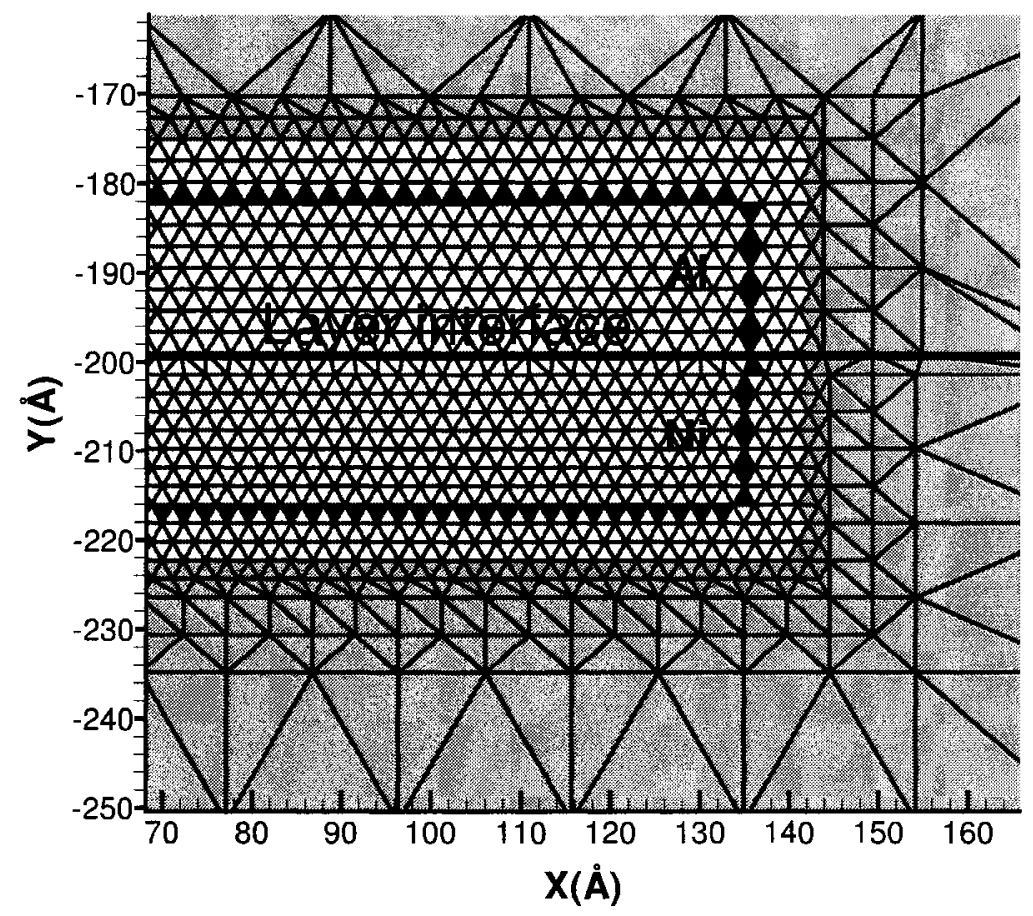

Figure 3.13: Position of the detection band (small black triangular elements) inside the second atomistic region. The grey elements are the continuum elements. 


\section{Chapter 4}

\section{Results and analysis}

\subsection{Bilayer simulation results}

As we have discussed in the previous chapter, the existing single grain CADD code is modified to observe the effects of nano-indentation into an Al-Ni bilayer system. Five different types of models are considered for bilayer simulation-

- Pure Aluminum single crystal

- Pure Nickel single crystal

- A $50 \AA$ aluminum layer placed on top of a nickel substrate of $480 \AA$ thick

- A $40 \AA$ nickel layer placed on top of a aluminum substrate of $490 \AA$ thick

- A $50 \AA$ nickel layer placed on top of a aluminum substrate of $480 \AA$ thick

In the first two cases, pure aluminum and pure nickel, are chosen as the materials for the entire region, i.e. these are single grain calculations to establish the baseline behavior of the pure materials. These two cases help us in determining the expected variation of the materials' response due to nano-indentation into bilayer systems. In the other three cases either a nickel or aluminum layer of varying layer thickness is placed on top of the other material.

In every model, the atoms/nodes that are located at the bottom surface are held fixed 
and a zero displacement boundary condition is applied. For rest of the atoms/nodes, a zero displacement constraint is applied only in the z-direction (the deformation is 2D). The indenter is allowed to move only in the y-direction. The upper surface is free, into which the indenter is pressed. The loading is quasi-static, and is achieved by displacement controlled nano-indentation. In this type of loading, the indenter position is moved down incrementally and a uniform incremental compressive strain is superimposed on the equilibrium solution from the previous load step. Then, the atoms, nodes, and continuum dislocations are allowed to relax while holding the indenter at a fixed position.

\subsubsection{Aluminum film on top of nickel substrate}

Aluminum has a lattice constant slightly larger than that of nickel. Thus, when an aluminum film is deposited on a nickel substrate (namely $\mathrm{Al} / \mathrm{Ni}$ ), this mismatch between two adjacent layers results in an array of misfit dislocations along the interface. Figure 4.1 shows the positions of the misfit dislocations (marked by points 1-9) along the interface for this case. These misfit dislocations play an important role to initiate the plastic deformation and also act as obstacles to glide the dislocation movement, at or near to the interface. Figure 4.2 to 4.5 , illustrate the deformation phenomena occurring at the atomistic region under the indenter at various stages of loading. The atoms are at the vertices of the small triangular elements, which are shown only to make visualization of the deformation easier. Figure 4.2 shows a close-up view of the misfit dislocations located at points 3-8 in Figure 4.1. As the indentation process progresses these misfit dislocations start to move and take new positions, points 3'-8' in Figure 4.2, along the layer interface. An important thing to be noticed here is that all these misfit dislocations move inward to the center of the model from their initial positions while indentation progresses. At certain stage of indentation one of these misfit dislocations (at point 6' in Figure 4.2) dissociates 


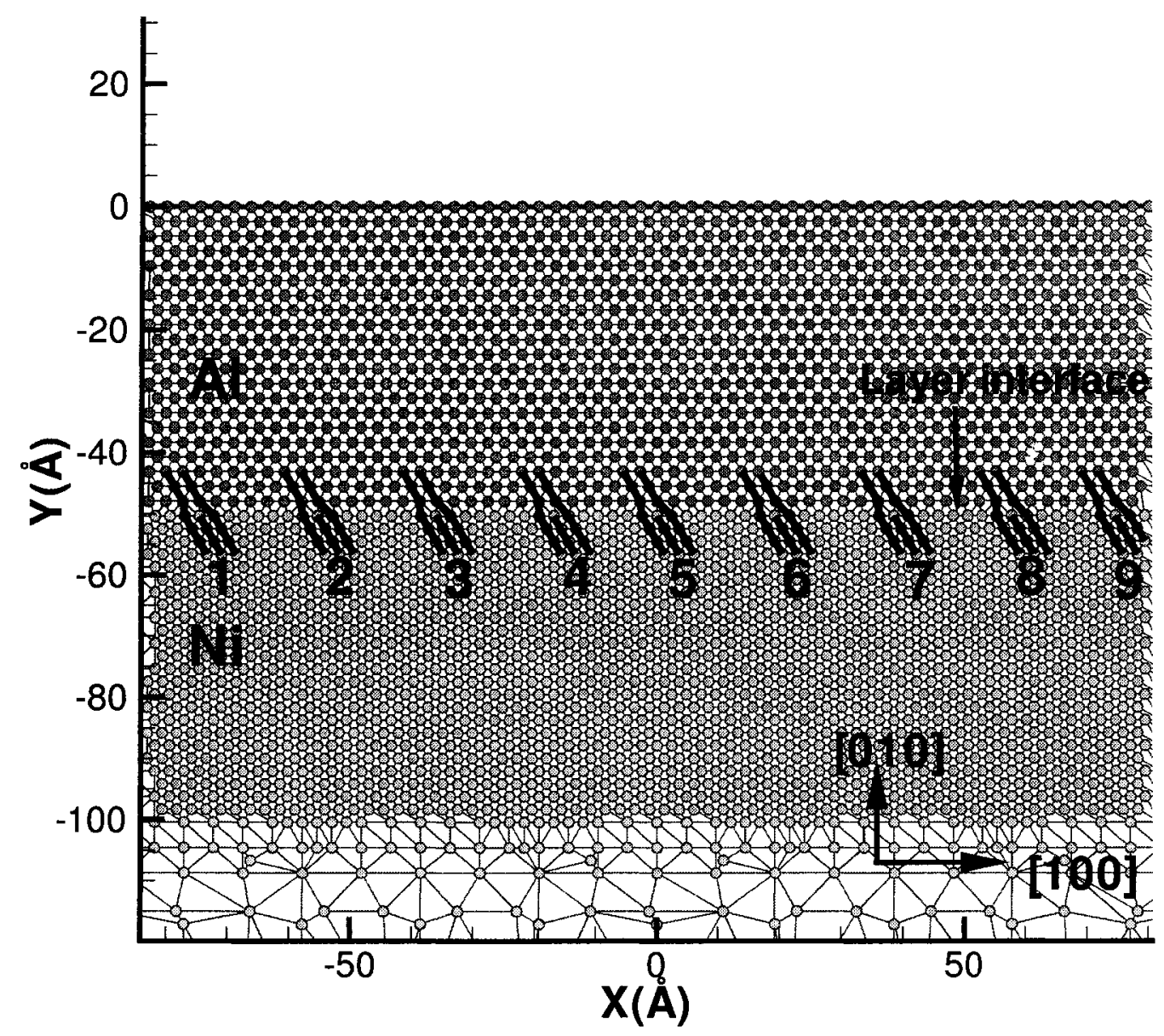

Figure 4.1: Positions of the misfit dislocations (point 1-9) along the interface due to the lattice constant mismatch between $\mathrm{Al}$ and $\mathrm{Ni}$.

and moves into the top aluminum layer. Right after the dissociation, two dislocations move upward along two slip planes and reach the surface of the top layer at point 'A' and 'B'. Figure 4.3 shows the dissociation of second dislocation also from the layer interface, point 4 ' in Figure 4.3, which also travels upward along two slip planes and reaches the top layer surface at point ' $\mathrm{C}$ ' and ' $\mathrm{D}$ '. The unique feature of the 2D hexagonal crystal structure is that, one dislocation gliding on one of the three slip systems can dissociates into two dislocations, one on each of the other two 


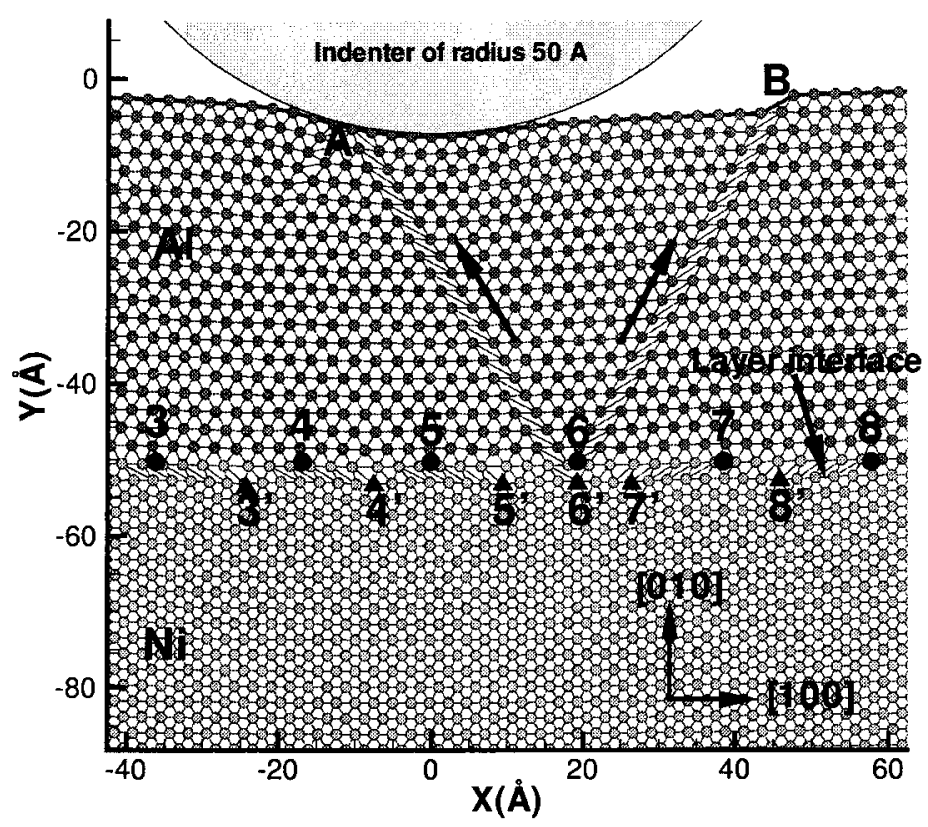

Figure 4.2: The primed (') positions are the final positions of the misfits. Dissociation of misfit starts from one of these positions (point $6^{\prime}$ ).

slip systems [14]. After reflecting back from the surface at point ' $\mathrm{A}$ ', the dislocation again dissociates into two new dislocations at point 'E', Figure 4.4. One of the new dislocations moves horizontally to the right and the other one moves down and to the right. As the indentation process progresses further, dislocations continue to dissociate from the layer interface and move to the aluminum layer. In addition to the dissociation activities from the interface, some dislocations also nucleate beneath the indenter inside the aluminum layer. But the thing to be noticed here is that all of the dislocation activities i.e., nucleation, dissociation and movement are confined within the aluminum layer instead of nickel during the whole indentation process, Figure 4.5. Since nickel is a harder material compared to aluminum, it is easier for a dislocation to move in aluminum than in nickel. 


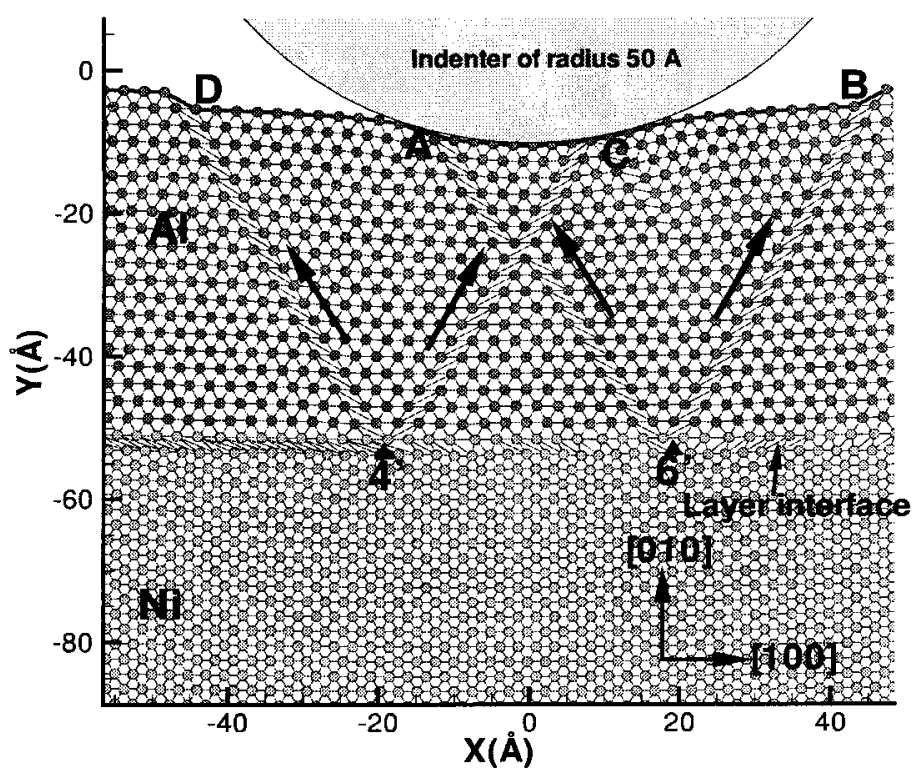

Figure 4.3: Dissociation of the misfit dislocations at different locations in the layer interface.

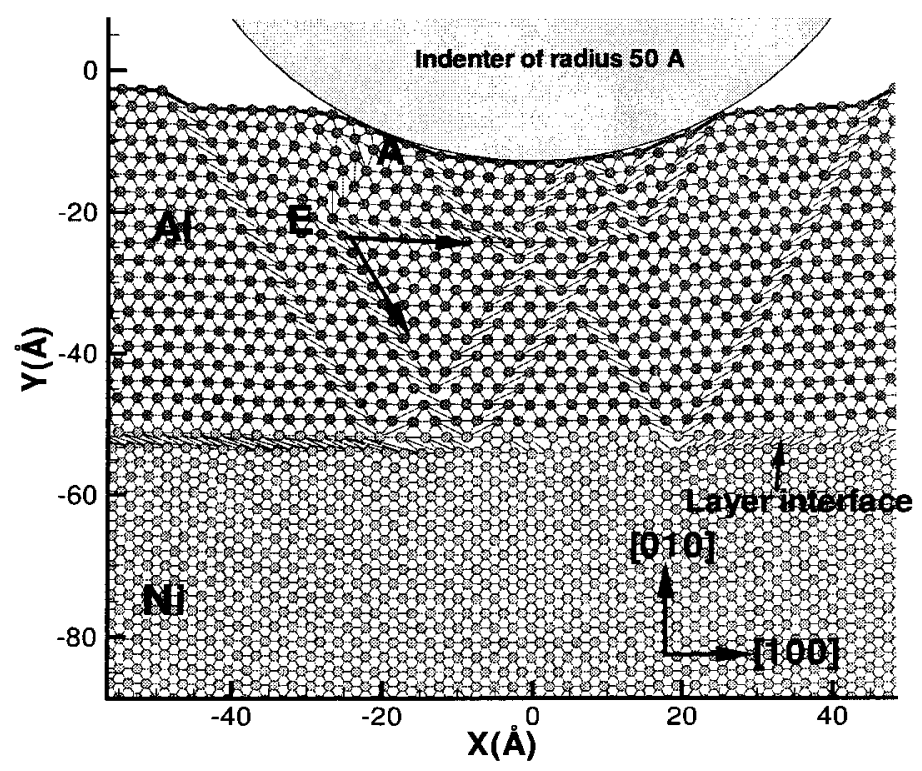

Figure 4.4: Dissociation of dislocation (at point E) after reflecting back from the indenter surface (point A). 


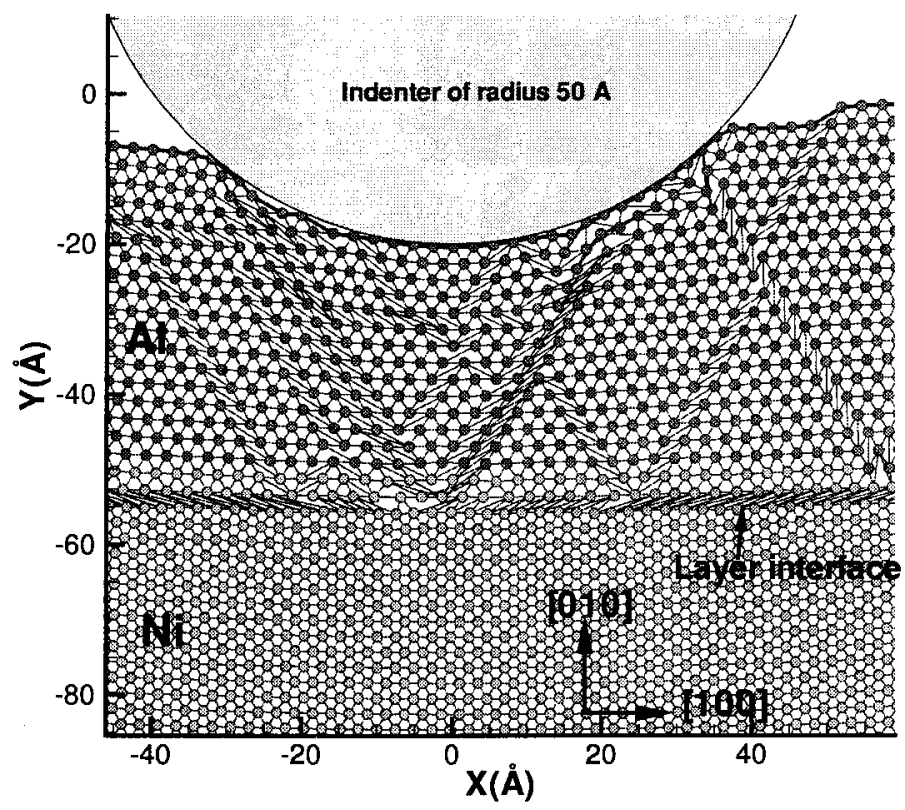

Figure 4.5: Accumulation of the dislocations in the aluminum layer.

\subsubsection{Nickel film on top of aluminum substrate}

A similar type of response is also found when a nickel layer is placed on top of an aluminum substrate (namely $\mathrm{Ni} / \mathrm{Al}$ ). Here also we notice the presence of misfit dislocations at the interface, marked by points 1-8 in Figure 4.6. Figure 4.7 show the initial and final positions of the misfits located between points $2-7$ respectively along the interface, which move outward from their initial positions. The opposite motion of the misfits in this case can be explained by the presence of dislocations of opposite sign, in Figure 4.6, to those in Figure 4.1 that form in the nickel layer due to its smaller lattice constant. Moreover, the stresses induced by the indenter also have a significant influence on the misfit dislocation movement and dissociation along the interface, which will be discussed in the following section. As in the $\mathrm{Al} / \mathrm{Ni}$ model, one of the misfit dislocations ( $b 1)$ dissociates ( $b 2$ and $b 3$ ) and moves along two slip planes into the lower aluminum layer. The misfit dislocation (b1) in Figure 


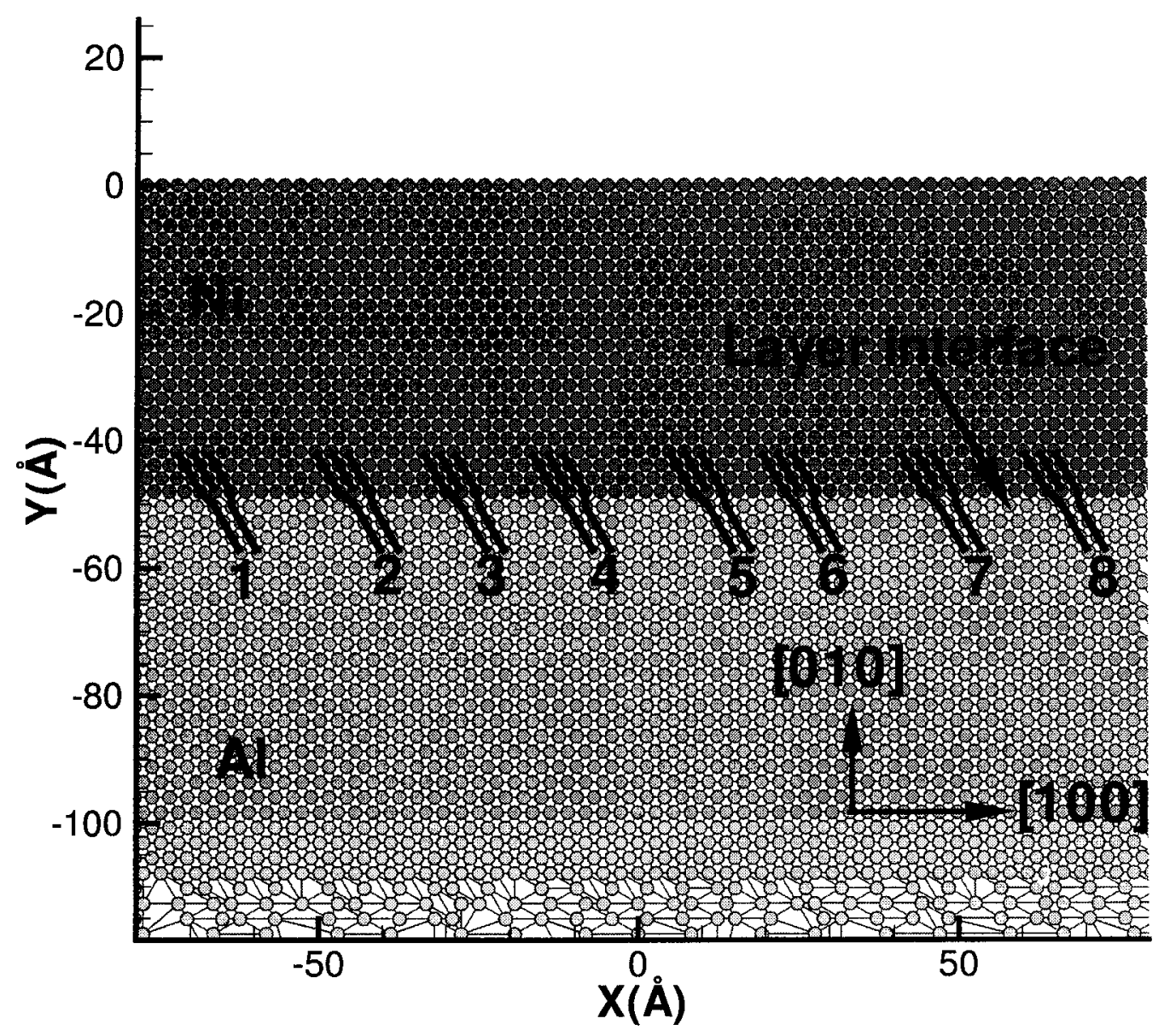

Figure 4.6: Position of the misfits along $\mathrm{Ni} / \mathrm{Al}$ interface. 


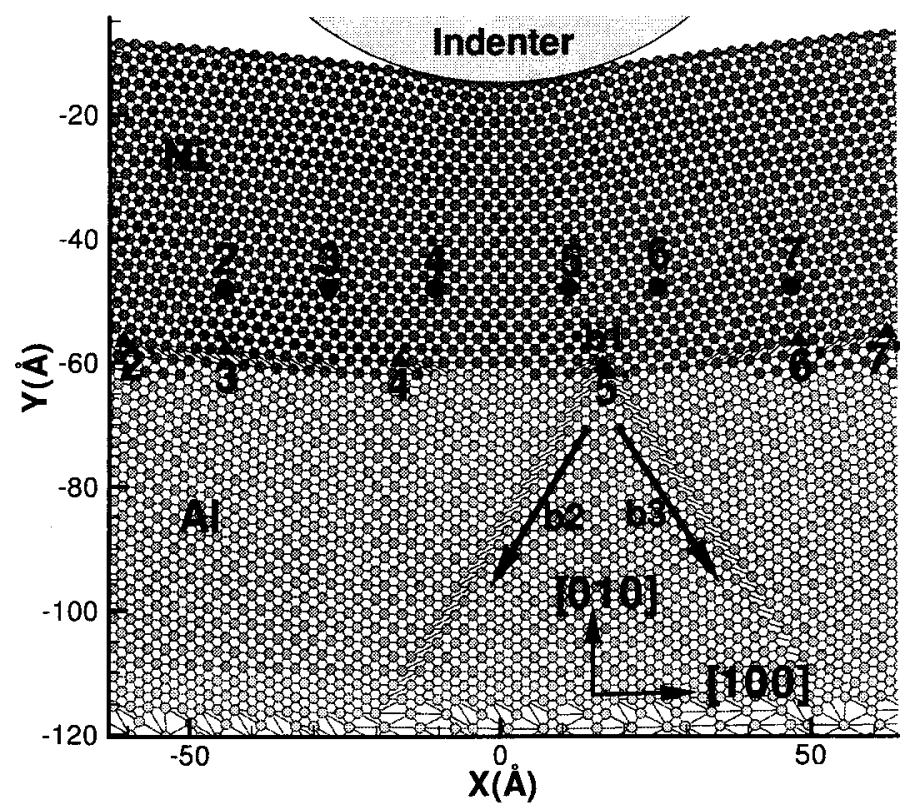

Figure 4.7: Movement of Misfit dislocations along the interface.

4.7 is found to be an edge dislocation whose Burgers vector is $[-2.766,0,0]$. The slip system of this dislocation can be written as: $[-\mathrm{a}, 0,0]\left(\begin{array}{ll}0 & 10\end{array}\right)$, where $\mathrm{a}=2.766 \stackrel{\AA}{\mathrm{A}}$, the lattice constant for Aluminum. The slip systems for the dissociated dislocations $b 2$ and $b 3$ can be written as:

$$
\begin{gathered}
b 2=[-1.383156,-2.395698,0.0](-1-10)=a / 2[-1,-\sqrt{3}, 0](-1-10) \\
b 3=[-1.383156,2.395698,0.0](1-10)=a / 2[-1, \sqrt{3}, 0](1-10)
\end{gathered}
$$

It is also observed that the misfit dislocations always move in the Al layer rather than in the Ni in both these cases, Figure 4.2 and Figure 4.7, which is quite expected due to the lower hardness of aluminum than nickel. While indenting further we also observe a few dislocations to nucleate in the nickel layer and then move to the aluminum layer across the layer interface, Figure 4.8. 


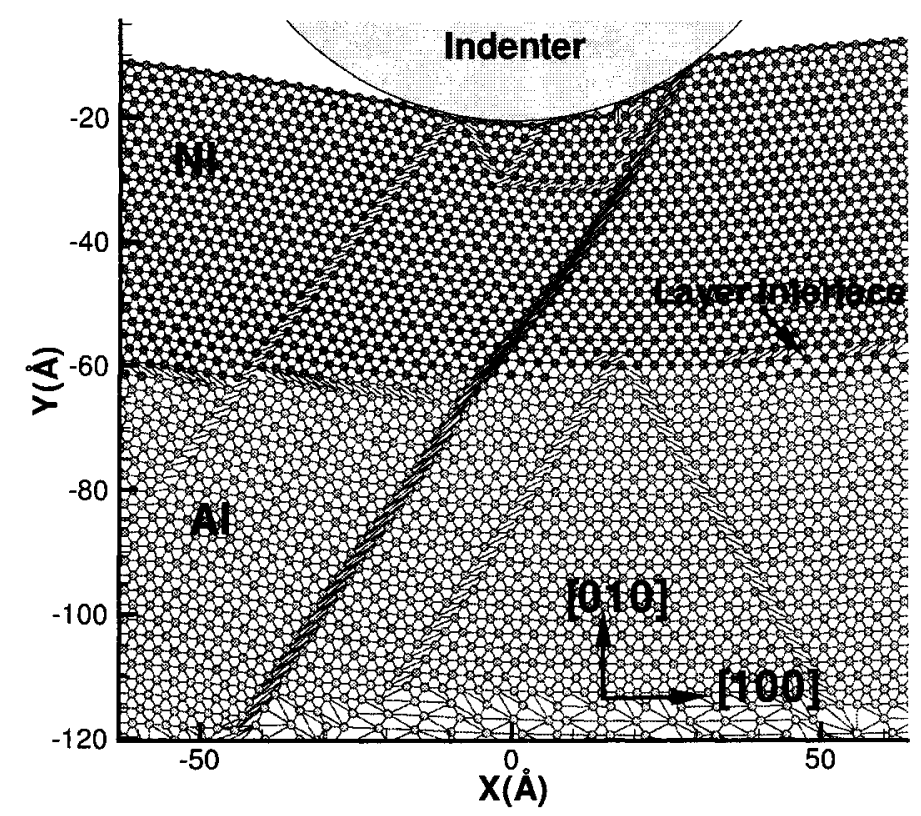

Figure 4.8: Passing of dislocation across the Ni/Al interface.

\subsubsection{Load-Displacement curves for Al-Ni bilayer systems}

Figure 4.9 presents the variation of load-displacement curves for these five cases. The maximum indentation depth varies from $20 \AA$ to $30 \AA$, which is approximately 50 percent of the film thickness for the bilayer models. At this large penetration depth it is not possible to determine film properties accurately without significant influence from the substrate. But since we are mainly interested about the dislocation nucleation and their interaction with the interfaces, we allow penetration as large as possible. In every case we find a complete elastic response of the system at the initial stage of loading, while the first drops in the curves indicate nucleation of the first dislocations (marked by points P1-P5 in Figure 4.9). The displacernent at which nucleation occurs and the corresponding load for every case are summarized in Table 4.1. This figure also clearly shows that the first dislocation nucleates at different level of load for the different cases. Since nickel is harder than aluminum, 


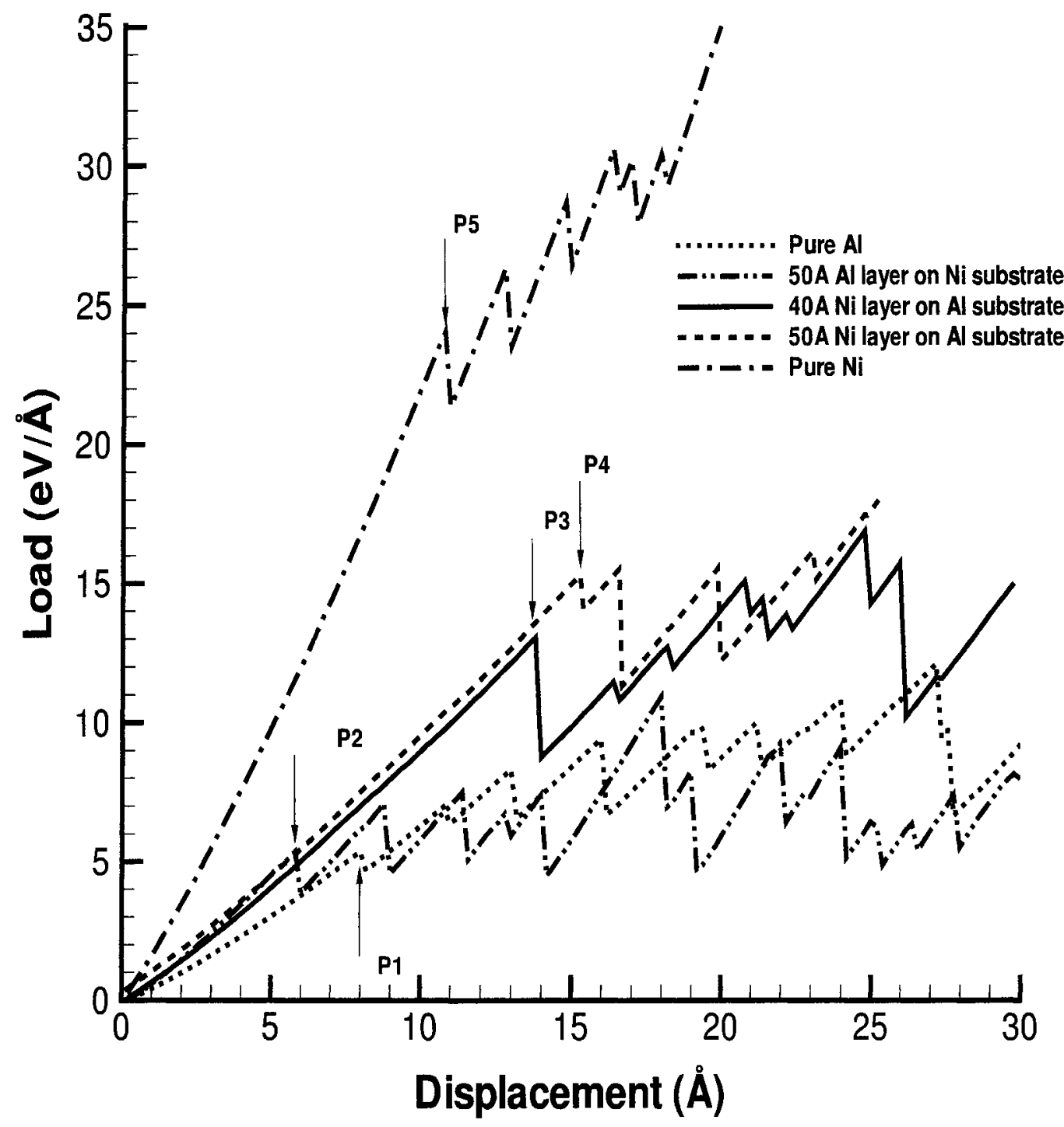

Figure 4.9: Load-Displacement curves for different Al-Ni bilayer system. For every case the overall thickness of the model is $530 \AA$.

the amount of load required to nucleate the first dislocation in nickel is much higher than that of aluminum, see Table 4.1. Similarly a nickel layer placed on top of an aluminum substrate also exhibit higher a load requirement to nucleate the first dislocation compared to an aluminum layer placed top of a nickel layer. 


\subsubsection{Hardness measurement for bilayer systems}

Load-displacement data are used to get information about the hardness variation with indentation depth, Figure 4.10. The hardness is measured by dividing the load by the contact area after every load step and the contact area at every load step is calculated by taking the projected area of the indented surface. In every case the hardness increases up to certain level of indentation depth and after that it decreases gradually or remains constant. Before reaching the maximum hardness point, there exist some fluctuations in the curve which have happened due to the quasi-static type of loading and sudden increase in contact area at certain load step. The maximum hardness obtained in each case is presented in Table 4.1. It is also clear from the figure that pure nickel is the hardest among the others and the aluminum layer placed on top of nickel shows the lowest hardness at most of the indentation depth. Though in the $\mathrm{Al} / \mathrm{Ni}$ case the bottom layer is of nickel, it still shows lower hardness than pure aluminum. The reason can be the bottom nickel layer acts like a rigid substrate, which impede any dislocation movement into it, thus, the overall response of this model is almost similar to the pure aluminum except that the aluminum layer thickness is less in this case. It can be concluded from this figure that by placing a thin layer of hard material on a substrate of soft material drainatically increases the overall hardness of the system and this hardness can be increased further by increasing the layer thickness. The hardness trends after first dislocation nucleation are shown in Figure 4.11. These trend lines are drawn only to smooth the hardness curves that will make visualization easier but can not be used to predict the materials' response beyond the point of maximum penetration depth for each case. According to our discussion in Chapter 2, hardening at nano-meter length scale occurs when large number of dislocations, confined over small volume, get tangled with each other and the movement of one dislocation is hindered by other dislocations and also by the interface. On the other hand, the indentation size effect leads to a 


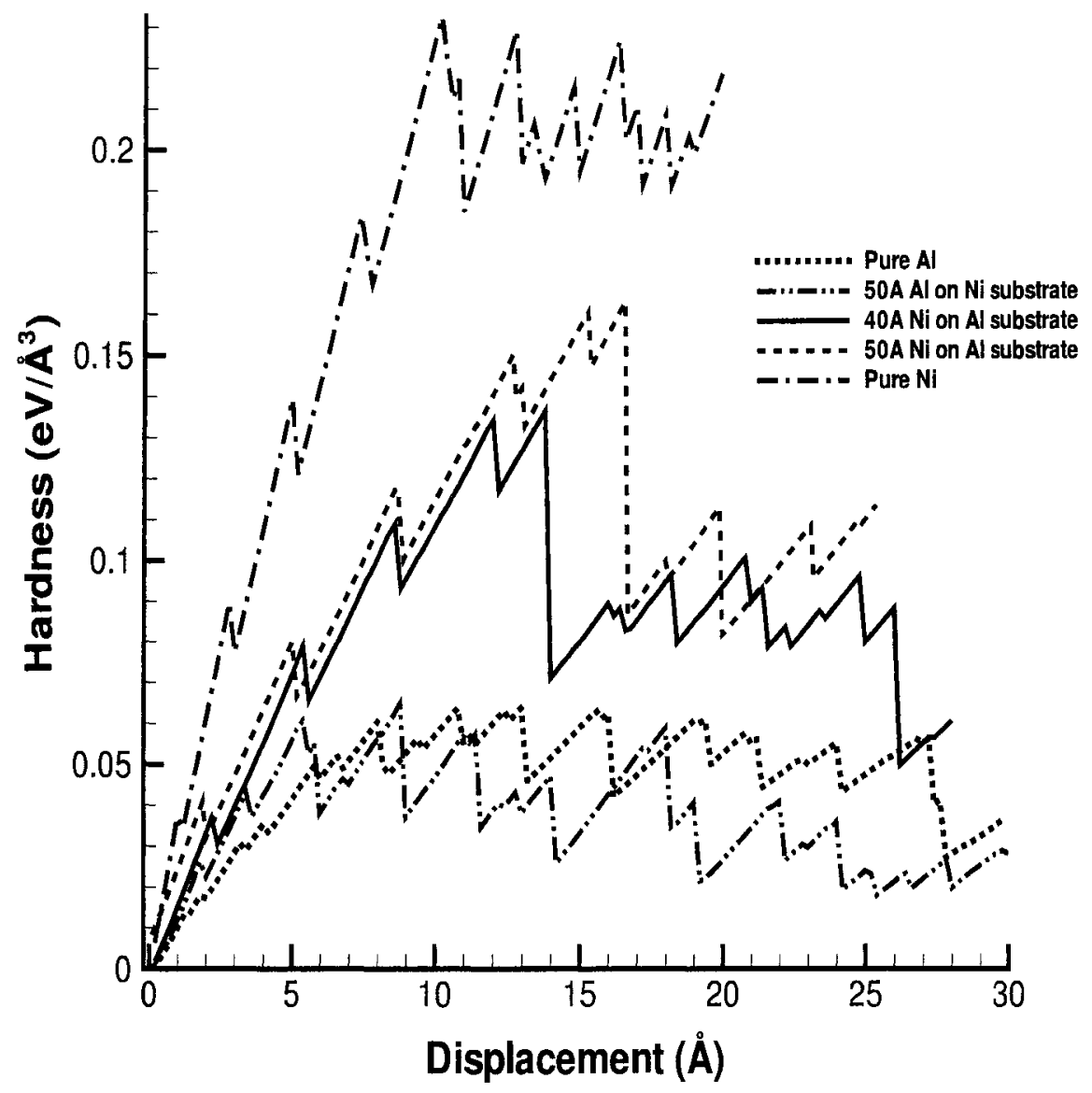

Figure 4.10: Hardness variation with depth of penetration for different bilayer models. For every case the overall thickness of the model is $530 \AA$. 
decrease in hardness at higher penetration depth due to the lower strain gradient. Thus, the hardness trends presented in Figure 4.11 are affected by simultaneous contributions of these two factors. These trends suggest that the indentation size effect is dominant in every case except for pure nickel, where the above two factors have equal contributions. The pure aluminum model (Figure 4.11(a)) behaves like a single crystal thick film bonded to a rigid substrate. The maximum penetration depth $(32 \AA)$ is much less than its overall thickness. Thus, there is no influence from the substrate. There exist large number of dislocations but they can spread over a large volume. Thus, hardening due to higher dislocation density is not present. But for an aluminum film on top of a nickel substrate model (Figure 4.11(c)), there exists a substantial influence of the nickel substrate. Up to $20-25 \AA$, hardness decreases steadily but after that, it seems to increase with penetration depth. As the dislocations fail to cross the $\mathrm{Al} / \mathrm{Ni}$ interface, a large number of dislocations accumulate within a small film volume and lead to increase in hardness at higher penetration depth. However, for other two cases (Figure 4.11(d) and (e)), where a nickel is placed on top of an aluminum substrate, even at maximum penetration depth (which is more than 50 percent of the film thickness), dislocations are quite mobile and do not experience any resistance from the interface. So, hardening due to increased dislocation density or dislocation-interface interaction is not observed. The movement of dislocations and the dissociation phenomena within single crystal pure $\mathrm{Ni}$ are not so abundant as those for single crystal pure Al. Moreover, unlike other bilayer models, the maximum indentation depth $\left(\delta_{\max }\right)$ is not that substantial (only 3.33 percent of the total bilayer thickness) to get any predominant effects of one of those two factors, rather the effects of hardening is almost mitigated by the indentation size effects at that small penetration depth.(Figure 4.11(b)). 


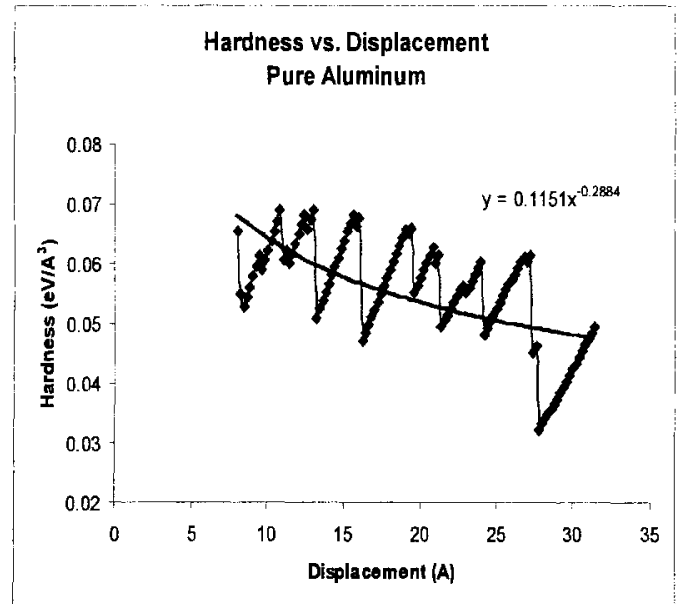

(a)

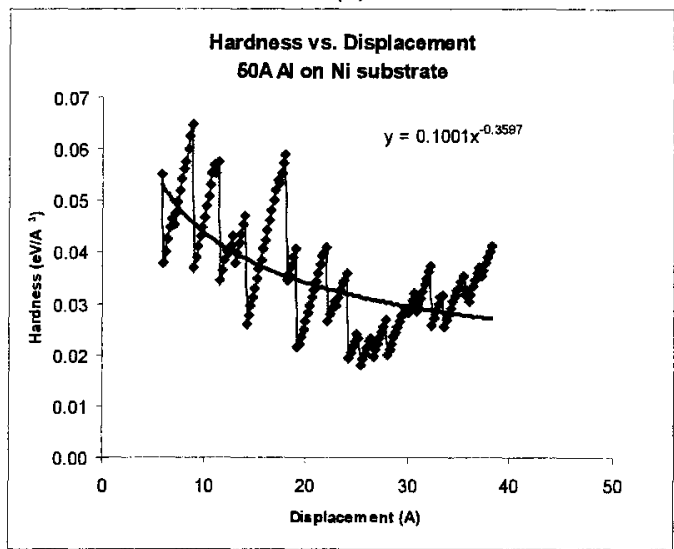

(c)

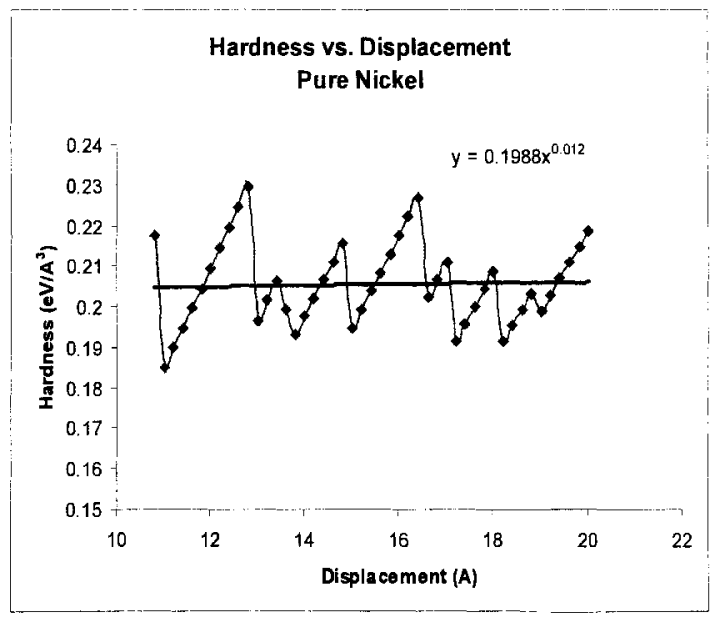

(b)

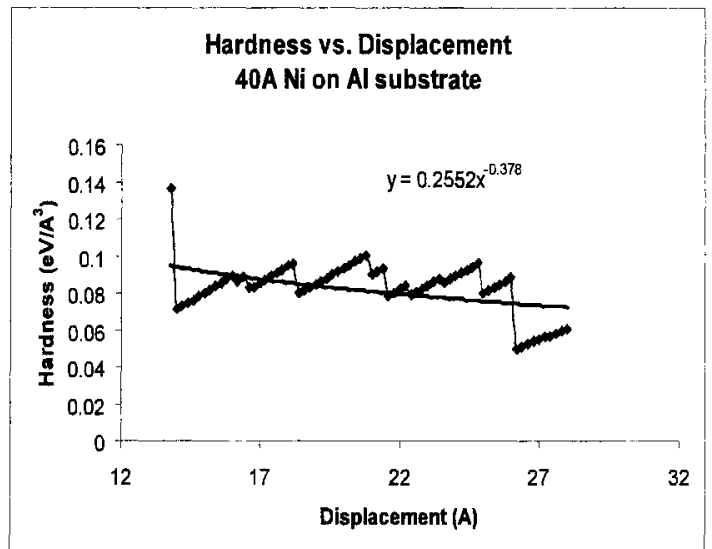

(d)

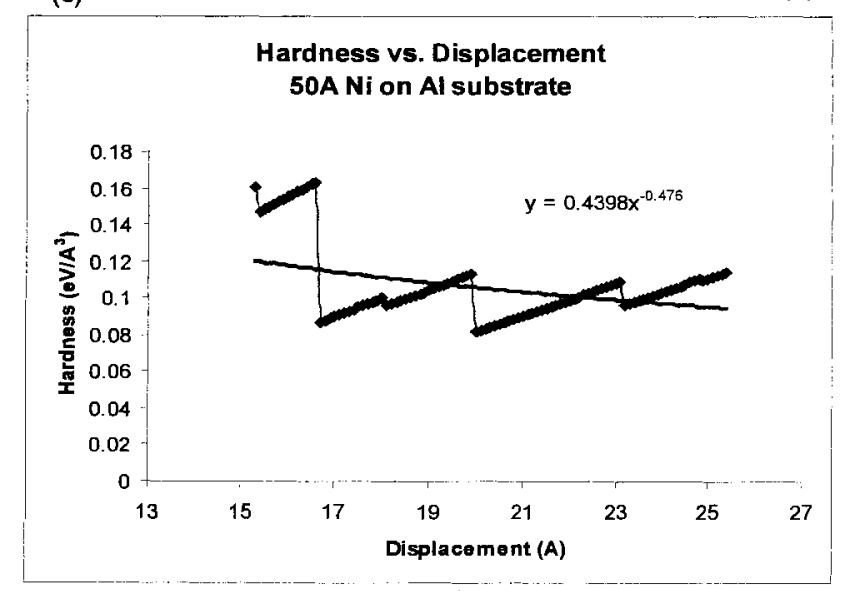

(e)

Figure 4.11: Hardness trends after first dislocation nucleation for different bilayer models. 


\begin{tabular}{|l|l|l|l|}
\hline Case & $\delta(\AA)$ & $P(\mathrm{eV} / \AA)$ & $H_{\max }(\mathrm{GPa})$ \\
\hline Pure $\mathrm{Al}$ & 8.0 & 5.3857 & 10.23 \\
\hline $50 \AA \mathrm{Al}$ on top of $\mathrm{Ni}$ & 5.8 & 5.3714 & 10.39 \\
\hline $40 \AA \mathrm{Ni}$ on top of $\mathrm{Al}$ & 13.8 & 13.074 & 21.88 \\
\hline $50 \AA \mathrm{Ni}$ on top of $\mathrm{Al}$ & 15.3 & 15.333 & 26.13 \\
\hline Pure $\mathrm{Ni}$ & 10.8 & 24.089 & 37.28 \\
\hline
\end{tabular}

Table 4.1: Summary of the results obtained from load vs. displacement curve and hardness vs. displacement curve for all bilayer models.

\subsubsection{Atomic level stress calculation}

To reveal the underlying mechanisms that initiate the movement and dissociation of the misfit dislocations along the interface, the atomic-level stresses are evaluated during the indentation process. The commonly known Virial theorem is used to evaluate these stresses, which for the EAM description of a material can be written as

$$
\sigma_{i}^{I J}=\frac{1}{\Omega_{i}} \sum_{j \neq i}\left[\Phi^{\prime}\left(r_{i j}\right)+F^{\prime}\left(\bar{\rho}_{i}\right) \rho^{\prime}\left(r_{i j}\right)+F^{\prime}\left(\bar{\rho}_{j}\right) \rho^{\prime}\left(r_{i j}\right)\right] \frac{r_{i j}^{I} r_{i j}^{J}}{r_{i j}}
$$

where, the superscripts $I$ and $J$ refer to Cartesian components, $\sigma_{i}^{I J}$ refers to the stress tensor at atom $i, r_{i j}^{I}$ is the $I^{\text {th }}$ component of the relative position vector between atoms $i$ and $j$, and $r_{i j}$ is its magnitude. $\Omega_{i}$ is the atomic volume at site $i . \Phi, F$ and $\rho$ are the pair potential, embedding energy and electron density respectively, while the prime components denote the differentiation with respect to $r_{i j}$ (see section 2.6.1 for more detail). Initially, when the indenter is well above the free surface, the indenter has no effect on the stress levels of the atoms. The atomic stresses are only the residual stresses that develop due to the interactions between different atoms during the relaxation process. Figure 4.12 shows the residual stress distribution of the atoms in the atomistic region. The interactions betwcen $\mathrm{Al}$ and $\mathrm{Ni}$ atoms across the interface can also be seen from this figure. When the indenter comes in contact with the free surface, the stress levels of the atoms start to change. To get the true 


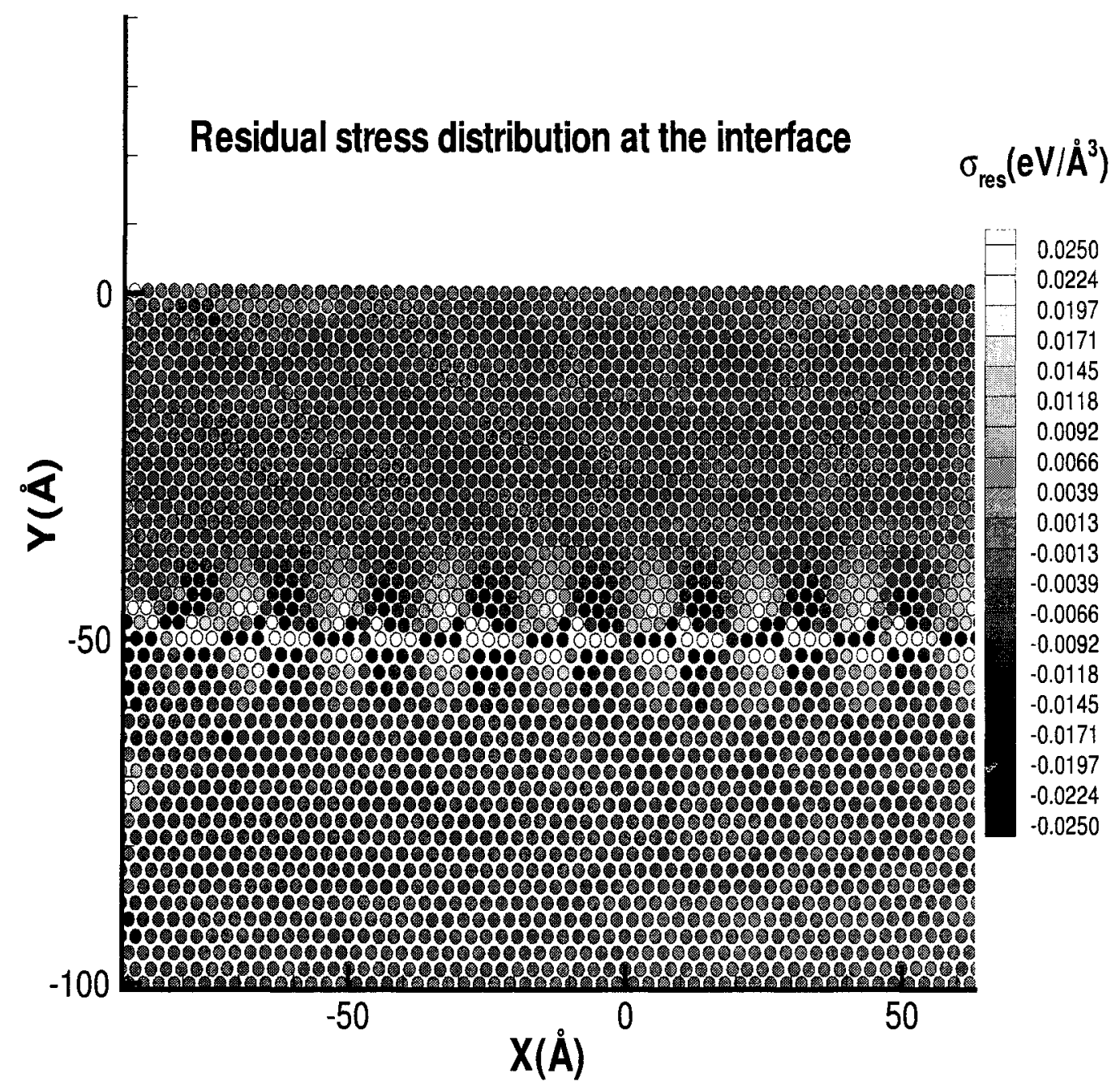

Figure 4.12: Residual stress distribution along the Ni/Al interface.

effect of the indenter, the residual stresses have been deducted from the total shear stress values of every atom. It has been found that during initial stages of loading, the indenter has no significant effect on the stress levels of most of the atoms except those located at the interface. Even when the indenter is just in contact with the interface (depth of penetration $0.3 \AA$ ), it alters the stress levels of the atoms located at the interface, Figure 4.13(a). The bright and dark colored zones in this figure are the points where the misfit dislocation cores are located. As the indentation 


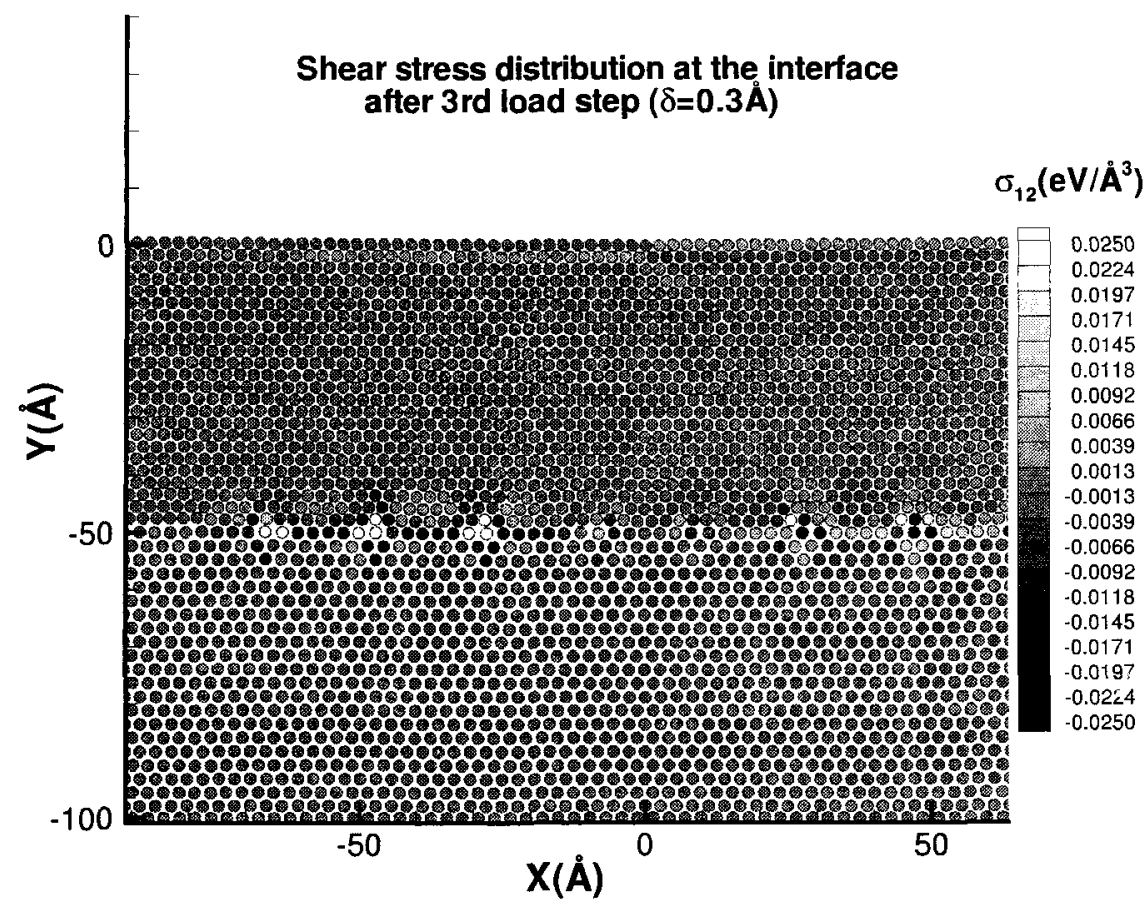

(a)

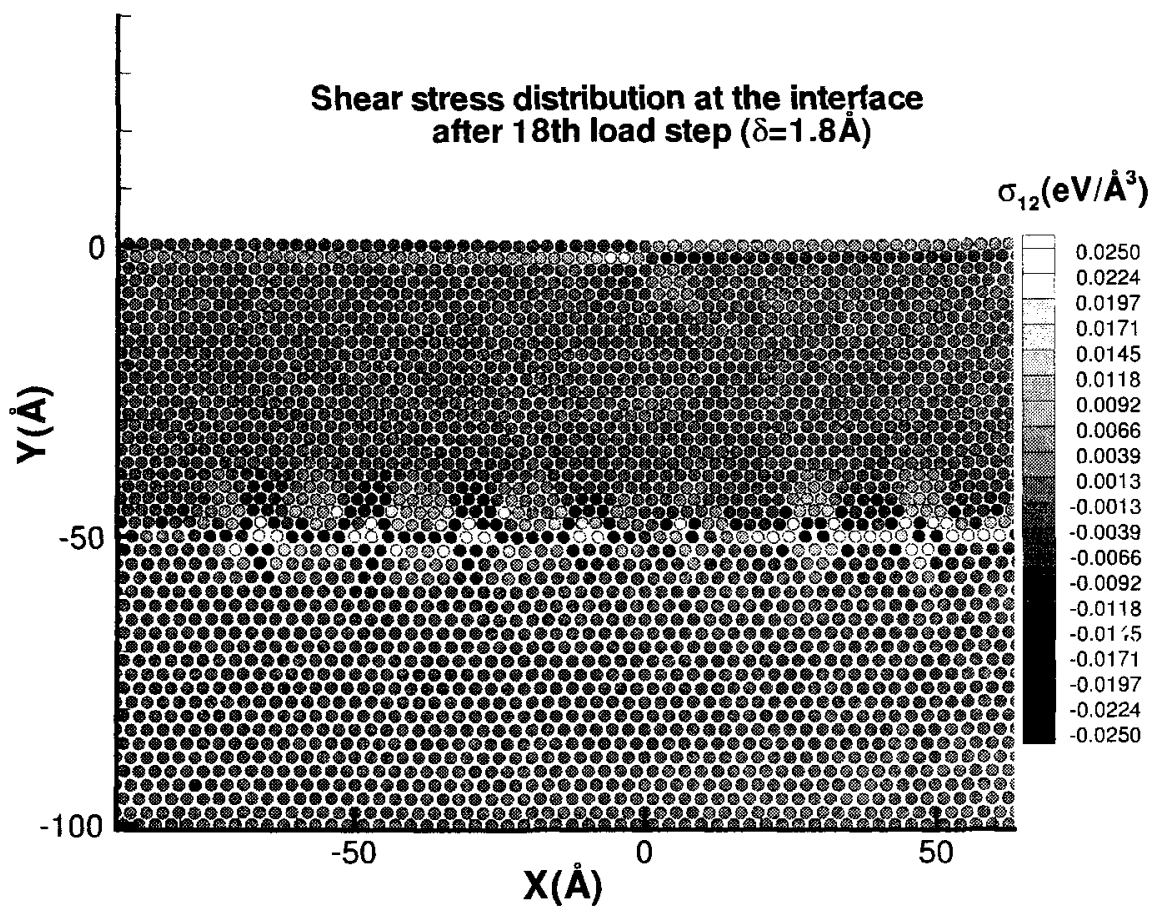

(b)

Figure 4.13: The effect of indenter on the atoms in the atomistic region at the indentation depth (a) $0.3 \AA$ and (b) $1.8 \AA$. 
progresses, the effect of the indenter at those locations on the interface becomes more significant, Figure 4.13(b), which gradually causes the dislocations to move along the interface and ultimately forces the dislocations to dissociate into the soft $\mathrm{Al}$ layer. The effect of the indenter on the stresses of surface atoms can also be seen in this figure. To get a more detailed picture about this phenomenon, the stresses of the interfacial atoms are plotted in Figure 4.14 and Figure 4.15. Figure 4.14, shows the shear stress variation of the atoms located at the interface $(Y=-50 \AA)$ after the 3rd and 18th load step. Two important things can be deduced from this figure. Firstly, as the indentation process progresses the effect of the indenter becomes

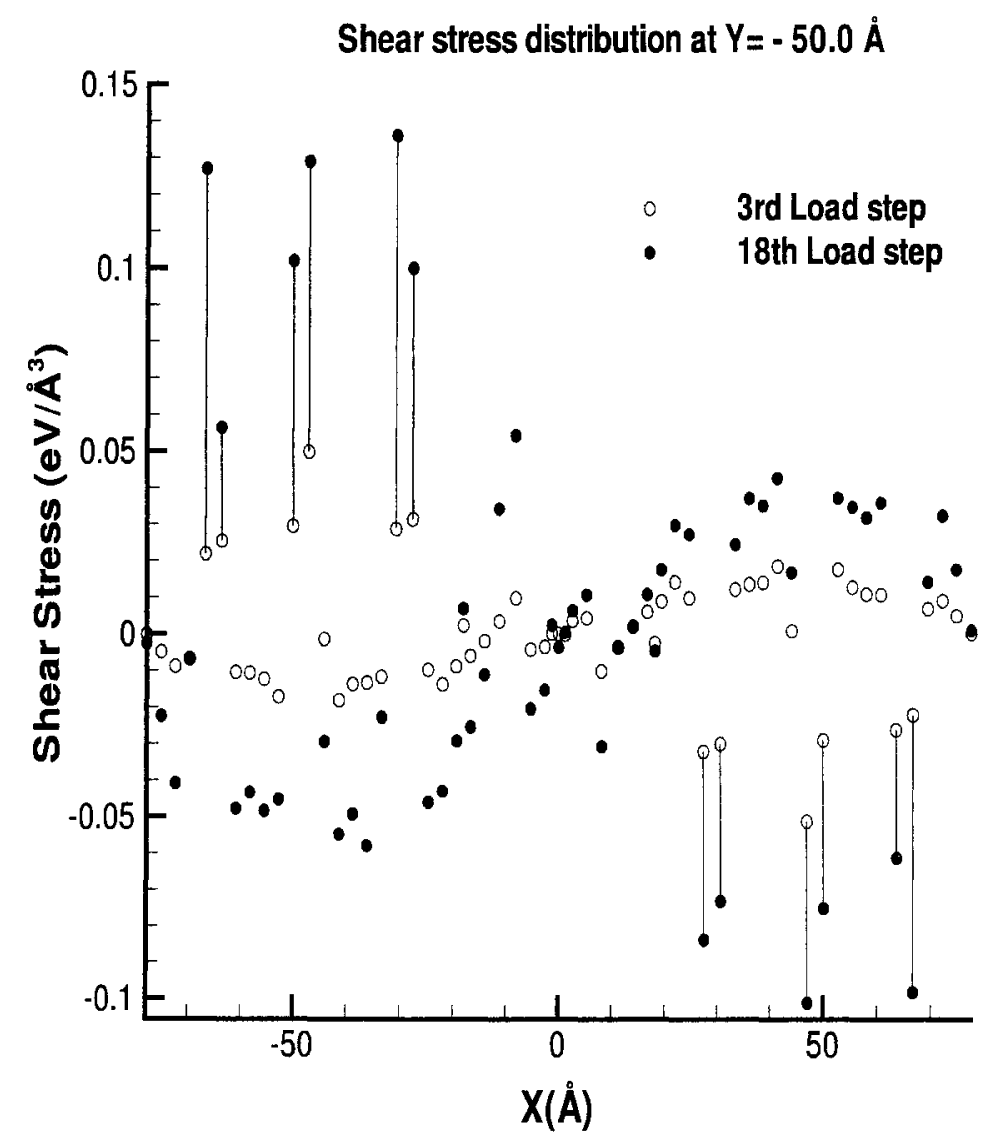

Figure 4.14: Shear stress variation of the atoms located at the interface $(Y=-50.0 \AA)$. The open circles are the stresses after 3rd load step and the filled circles are the stresses after 18th load step. 


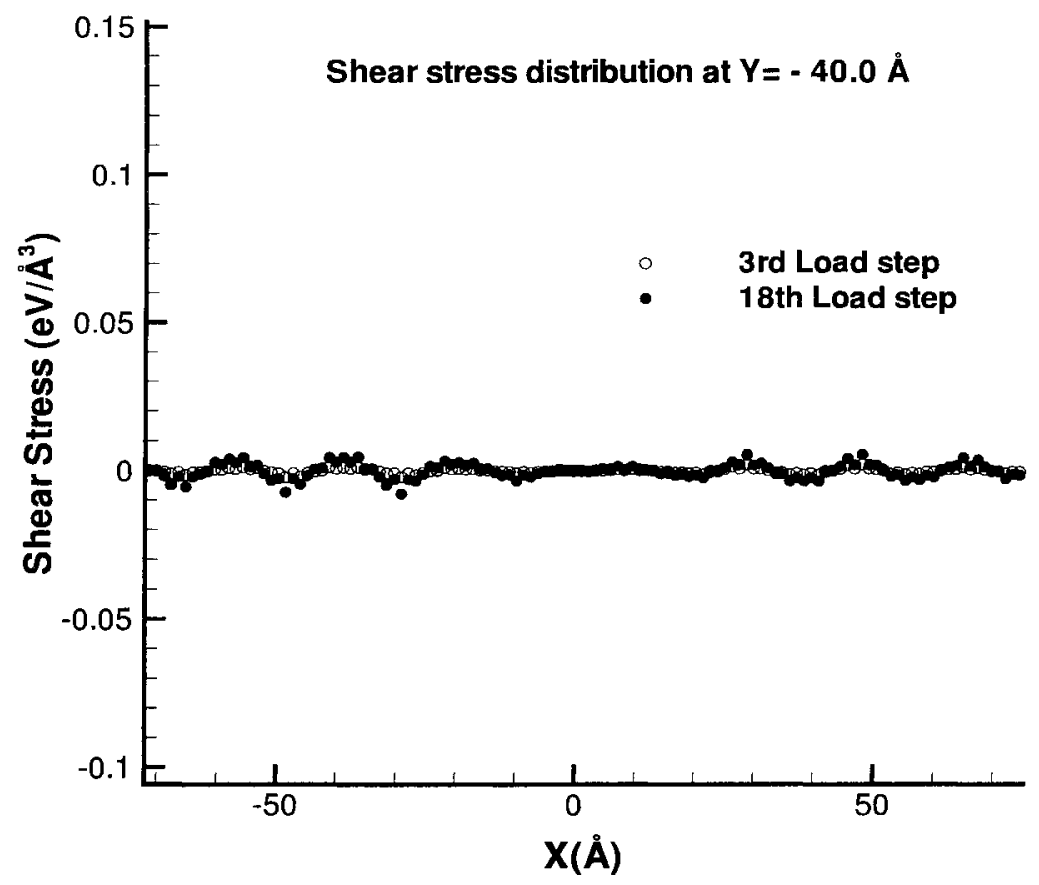

(a)

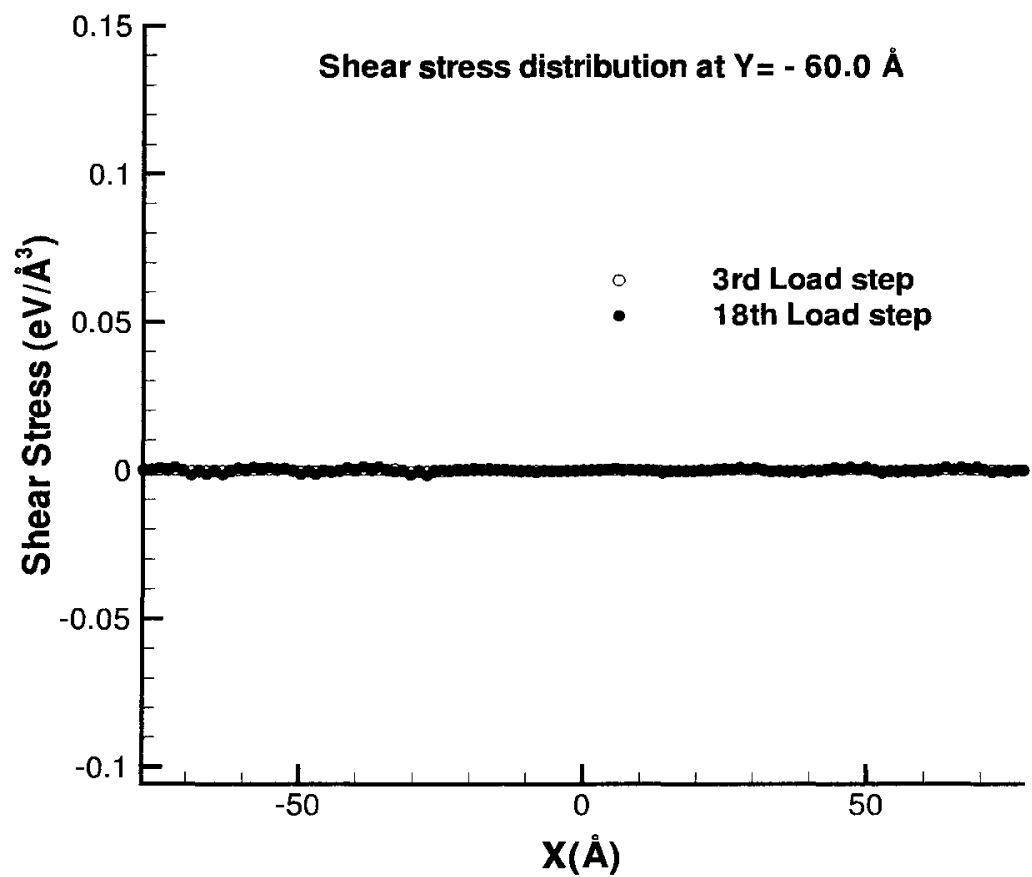

(b)

Figure 4.15: Shear stress variation of the atoms located (a) $10.0 \AA$ above the interface $(Y=-40.0 \AA)$ and (b) $10.0 \AA$ below the interface $(Y=-60.0 \AA)$. The open circles are the stresses after $3 r d$ load step and the filled circles are the stresses after 18th load step. 
more prominent at the positions where the misfits are located. The large variation of stresses at those positions can also be seen from this picture. Secondly, the stress levels at the same locations on the either side of the center of the model are opposite to each other. Though the magnitudes are not the same but the opposite nature of the stresses results in opposite motion of the misfit dislocations along the grain boundary. As it has been discussed before, the motion can either be inward or outward depending upon the film-substrate system. As we move further from the interface, either top or bottom, the effect of the indenter becomes less significant. Figure 4.15(a) and Figure 4.15(b) show the stress variation of the atoms located $\pm 10 \AA$ from the interface. No noticeable variations are observed at those positions.

\subsection{Multilayer simulation results}

After simulating the bilayer models, the CADD code has been modified further to study an Al-Ni multilayer structure, and its simplest case with two layers of films coated on a substrate is modelled. Thin layers of $\mathrm{Al}$ and $\mathrm{Ni}$ films on either $\mathrm{Al}$ or $\mathrm{Ni}$ substrate are considered:

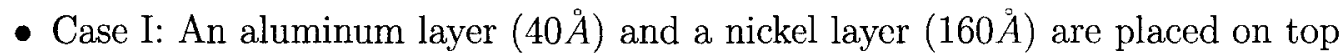
of an aluminum substrate ( $400 \AA$ ) (namely $\mathrm{Al}-\mathrm{Ni}-\mathrm{Al}$ system).

- Case II: A nickel layer (40 $\AA$ ) and an aluminum layer $(160 \AA)$ are placed on top of a nickel substrate $(400 \AA)$ (namely Ni-Al-Ni system).

- Case III: Ni-Al-Ni system but without atomistic region at the second interface (namely Ni-Al-Ni without 2 nd atomistic).

The behavior of these models are compared with the behaviors of the pure metals of the same model size. 


\subsubsection{Ni-Al-Ni system}

Figure 4.16 shows the deformed configuration of the Ni-Al-Ni system due to the nano-indentation. Any dislocation, that nucleates in the top Nickel film, is able to cross the first interface quite easily, and shows easy glide along the soft aluminum layer. But when it comes down to the second interface, its movement is blocked due to the presence of hard $\mathrm{Ni}$ substrate. Moreover, the misfit dislocations present at the interface also incorporate extra resistance to the dislocation motion. Figure 4.16 shows, one of the dislocations, nucleated in the top layer, point A in Figure 4.16, hits one of the misfits present at the second interface, point B in Figure 4.16 and triggers it to be dissociated into the $\mathrm{Al}$ layer, point $\mathrm{C}$ in Figure 4.16. As we modeled this portion of the interface with the atomistic region, both the lattice constant mismatch and the elastic constant mismatch between adjacent layers contribute to the blocking of dislocation movement.

\subsubsection{Ni-Al-Ni system (without an atomistic region at the second interface)}

The necessity of the atomistic region near the interface can be justified by modeling the previous case but replacing the second atomistic region with the finite elements. Thus, we intentionally ignore the atomistic details (i.e. misfit dislocations, stacking faults etc.) at the second interface. Figure 4.17 shows the deformed configuration of this model due to the indentation, which is slightly different than that of the previous case. As before, any dislocation that nucleates in the top layer can cross the first interface very easily. But, instead of being blocked at the second interface, it also crosses the second interface and eventually yields the hard Ni substrate, Figure 4.17. The result suggests that glide dislocations do not experience significant resistance while crossing the interface if only the elastic constant mismatch exists between the adjacent layers. Thus, the continuum model is not sufficient to predict 


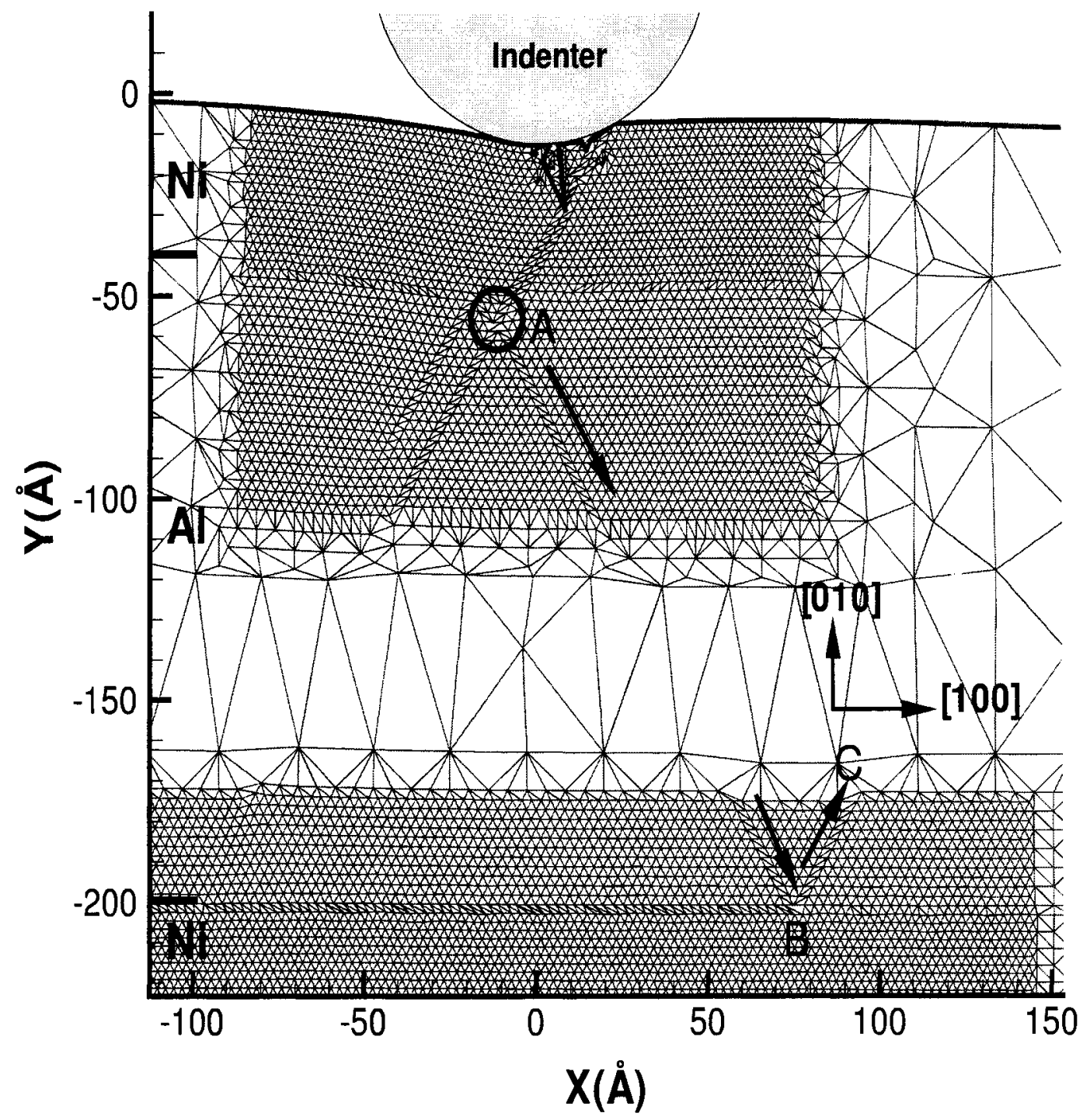

Figure 4.16: Deformation configuration of Ni-Al-Ni model. 


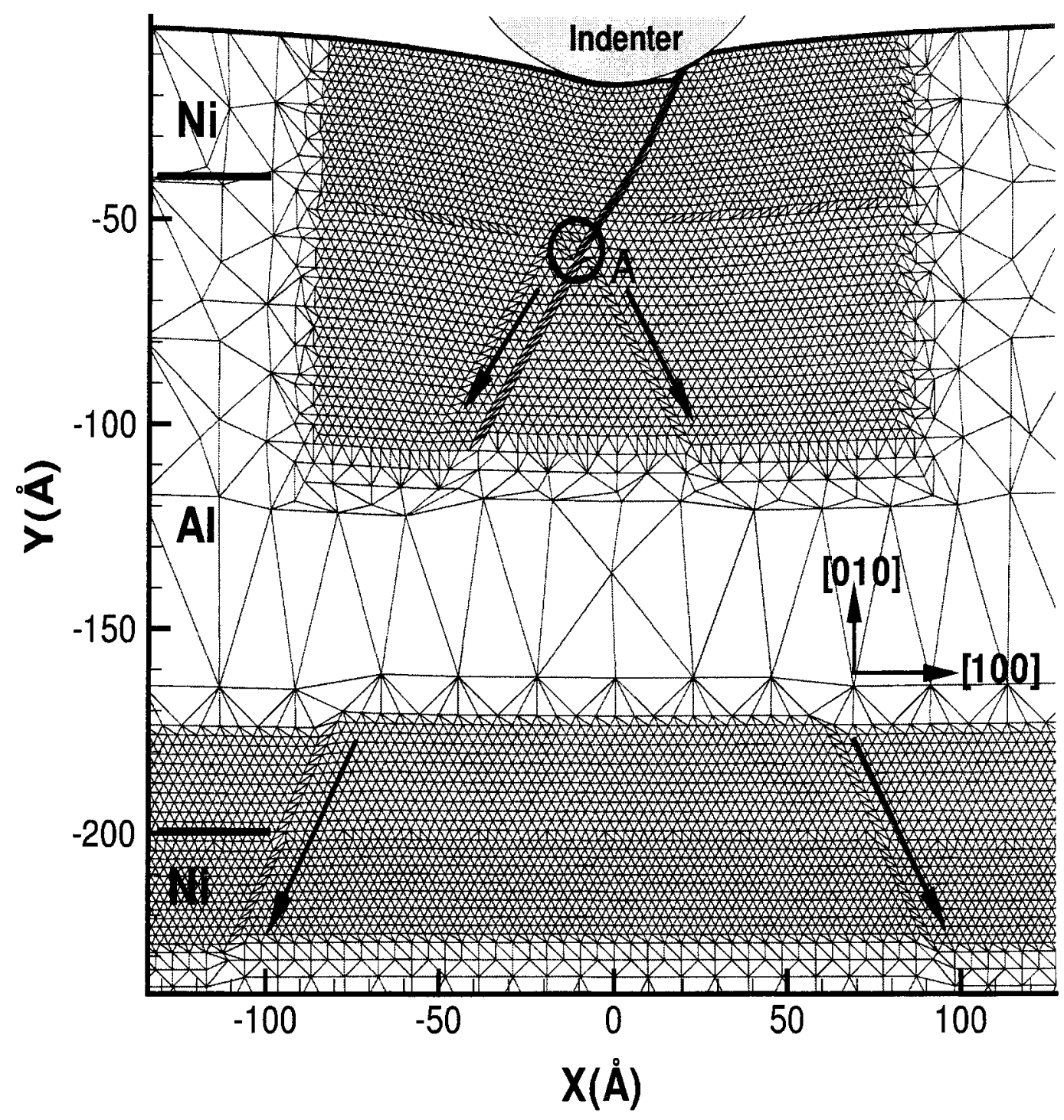

Figure 4.17: Deformation configuration of $\mathrm{Ni}-\mathrm{Al}-\mathrm{Ni}$ (without 2nd atomistic region) model.

the behavior exactly and requires the necessity of atomistic models at the locations where dislocation/interface interactions are possible. 


\subsubsection{Load-Displacement curves for $\mathrm{Ni}$-Al multilayer sys- tems}

Figure 4.18 shows a plot of load vs. displacement for all of the five cases. Like the bilayer models, all multilayer models show a good elastic response at the initial stage of loading. The first dislocation nucleation points are marked by points P1-P5 in Figure 4.18 for different cases. The amount of load required to nucleate the first dislocation $(P)$, the corresponding penetration depth $(\delta)$ and the amount of load drop $(\Delta P)$, and maximum hardness obtained $\left(H_{m a x}\right)$ for all cases are summarized in Table 4.2 .

Pure Ni model exhibits the highest load requirement $(24.869 \mathrm{eV} / \AA$ at penetration depth of $11.5 \AA$ ), whereas, $\mathrm{Al}-\mathrm{Ni}$ - $\mathrm{Al}$ models shows the lowest load requirement $(4.7323 \mathrm{eV} / \AA$ at penetration depth of $6.7 \AA$ ) to nucleate the first dislocation, which is also less than that of the pure aluminum model $(6.5132 \mathrm{eV} / \AA)$. The other two models, Ni-Al-Ni and $\mathrm{Ni}-\mathrm{Al}-\mathrm{Ni}$ (without the 2nd atomistic), have almost similar $P-\delta$ curves until the first dislocation nucleates (points P2 and P3 in Figure 4.18). The amount of load required and the indentation depth are almost the same but the amount of load drop at those points are quite different. A higher load drop of $5.2477 \mathrm{eV} / \AA$ in the Ni-Al-Ni system, compared to $2.716 \mathrm{eV} / \AA$ for $\mathrm{Ni}-\mathrm{Al}-\mathrm{Ni}$ (without 2nd atomistic) model, corresponds to the nucleation of dislocation at the top layer, as well as the dissociation of the misfit dislocation at the second interface. All the plastic deformations are confined only at the top film for $\mathrm{Al}-\mathrm{Ni}-\mathrm{Al}$ model, due to the resistance imposed by the hard second layer at the first interface. But for the Ni-Al-Ni model, dislocations are able to yield both the films but not the substrate. However, for $\mathrm{Ni}-\mathrm{Al}-\mathrm{Ni}$ (without 2nd atomistic) model, the substrate has also been yielded, as the second interface fail to block the dislocation movement. 


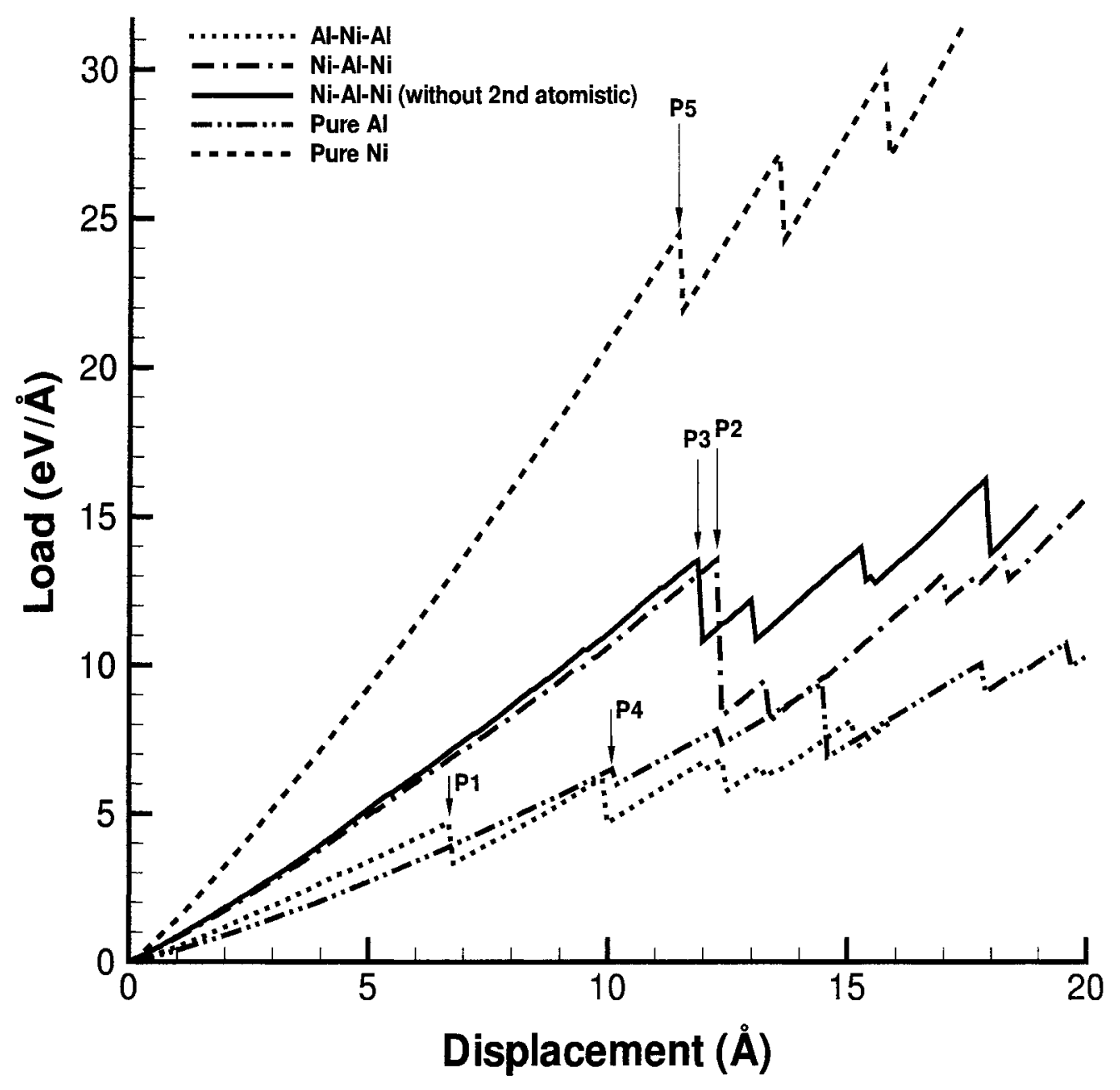

Figure 4.18: Load-Displacement curves for different Al-Ni multilayer system. 


\subsubsection{Hardness measurement for multilayer systems}

Figure 4.19 shows the hardness vs. displacement plots for all of the multilayer models. Pure Ni exhibits the highest hardness of $36.82 \mathrm{GPa}$ (see Table 4.2) compared to the other models. Hardness values of Ni-Al-Ni model and Ni-Al-Ni(without 2nd atomistic) model are almost the same but less than that of the pure Ni due to the presence of soft $\mathrm{Al}$ layer in the middle. Hardness of Al-Ni-Al system is almost the same as pure $\mathrm{Al}$ since all the plastic deformations occur in the top aluminum layer. Figure 4.20 shows the hardness trends after nucleation of first dislocation for three $\mathrm{Al}-$ Ni multilayer models. For the Al-Ni-Al model, Figure 4.20(a), the hardness remains unchanged as the indentation progresses and thus, there is no clear evidence of a hardening or the indentation size effect. This behavior is similar to that obtained in single crystal thin film results [14]. The hardness in this case is undoubtedly affected by the close influence of a hard second layer of Ni. The hardness trend for the Ni-Al-Ni model, Figure 4.20(b), can be said to be free from any influence of the substrate but there exists a good influence of the interface in determining the model's properties. We observe a hardening effect due to dislocation blocking at the second interface, although the rise is not very sharp. This is because the hardening is mitigated by simultaneous indentation size effect. In the Ni-Al-Ni (without 2nd atomistic) model, Figure 4.20 (c), we do not see any dislocation blocking at any interface, so no hardening occurs here. As such, we only see the indentation size effect in the last model as the indentation progresses. 


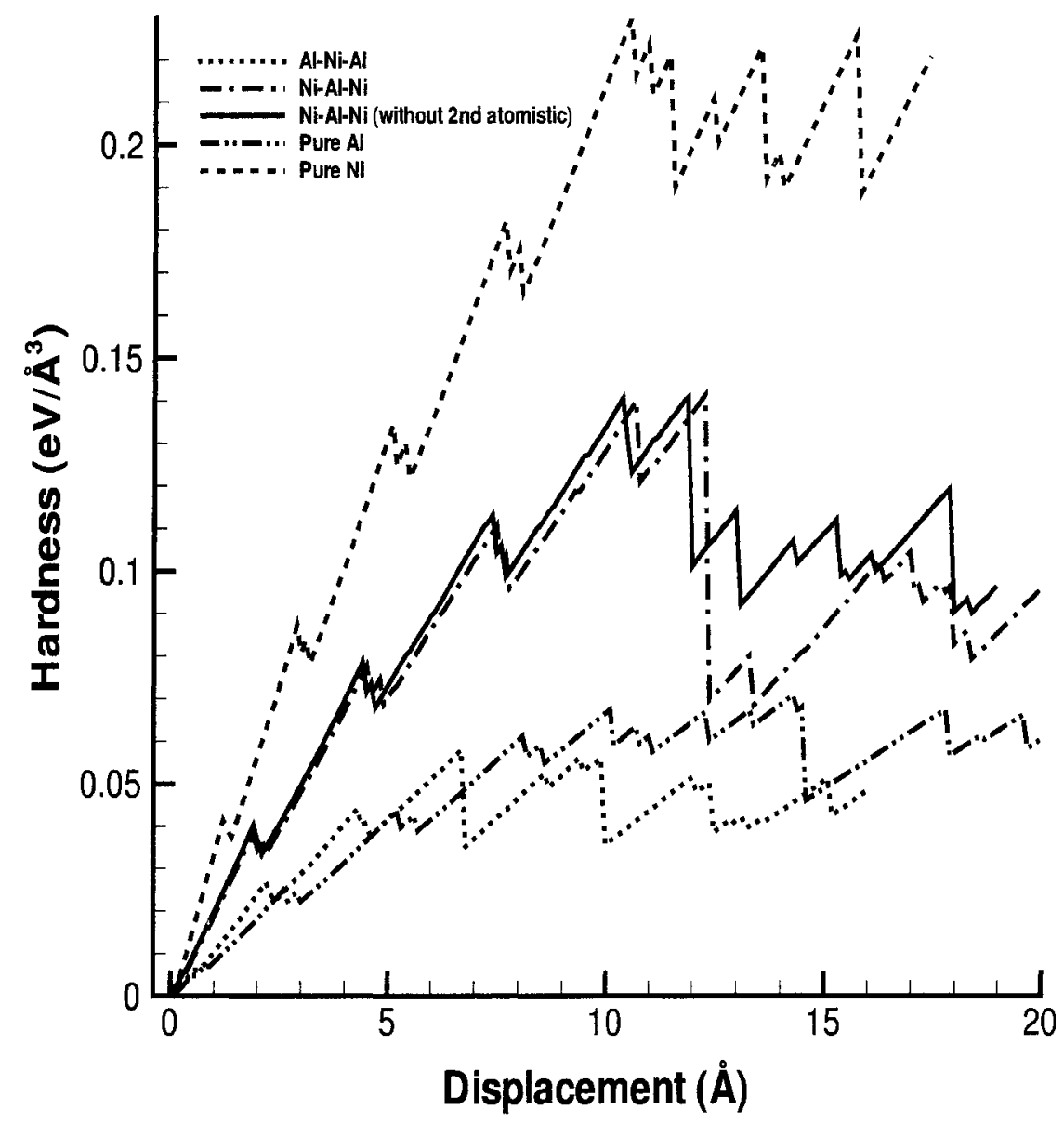

Figure 4.19: Hardness variation with depth of penetration for different multilayer models.

\begin{tabular}{|l|l|l|l|l|l|}
\hline Case & $\delta(\AA)$ & $P(\mathrm{eV} / \AA)$ & $\Delta P(\mathrm{eV} / \AA)$ & $H_{\max }(\mathrm{GPa})$ & Deformed layer \\
\hline Al-Ni-Al & 6.7 & 4.7323 & 1.3941 & 9.31 & top film only \\
\hline Ni-Al-Ni & 12.3 & 13.559 & 5.2477 & 22.66 & both of the films \\
\hline Ni-Al-Ni & 11.9 & 13.526 & 2.716 & 22.57 & $\begin{array}{l}\text { both films and } \\
\text { the substrate }\end{array}$ \\
\hline Pure Al & 10.1 & 6.5132 & 0.5064 & 11.34 & Not applicable \\
\hline Pure Ni & 11.5 & 24.869 & 2.523 & 36.82 & Not applicable \\
\hline
\end{tabular}

Table 4.2: Summary of the results obtained from load vs. displacement curve and hardness vs. displacement curve for all multilayer models. 


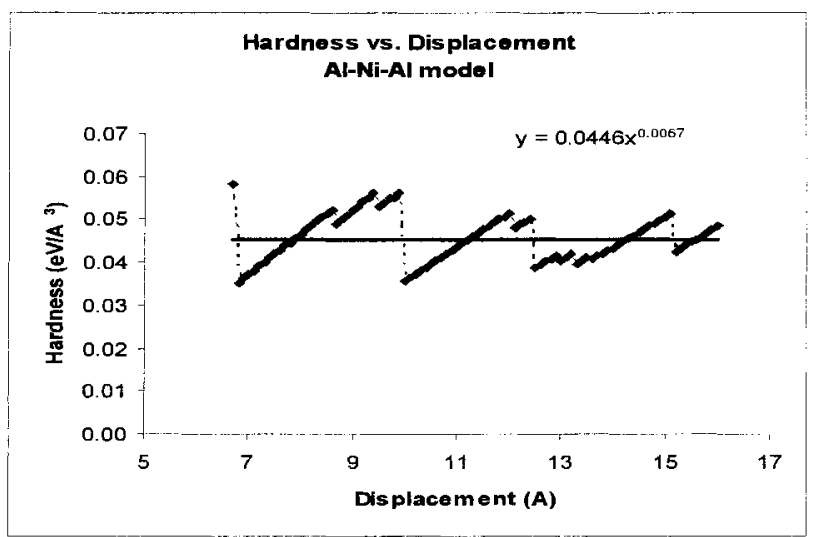

(a)

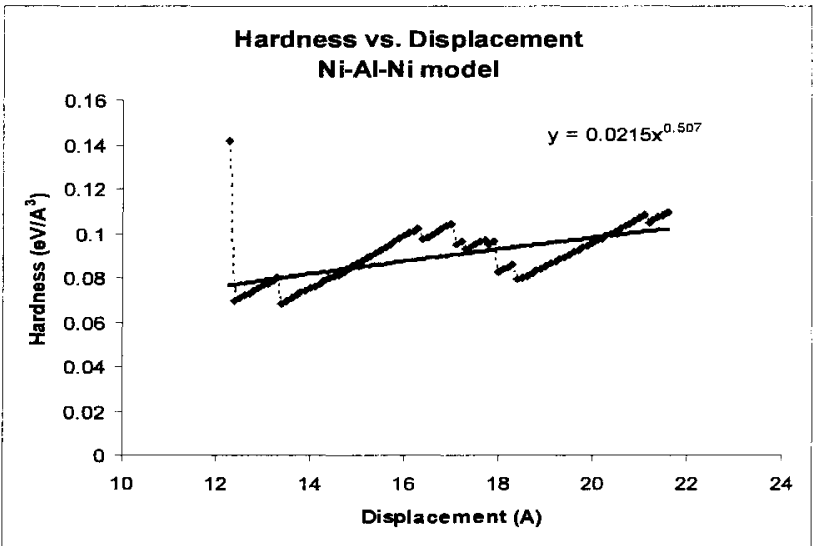

(b)

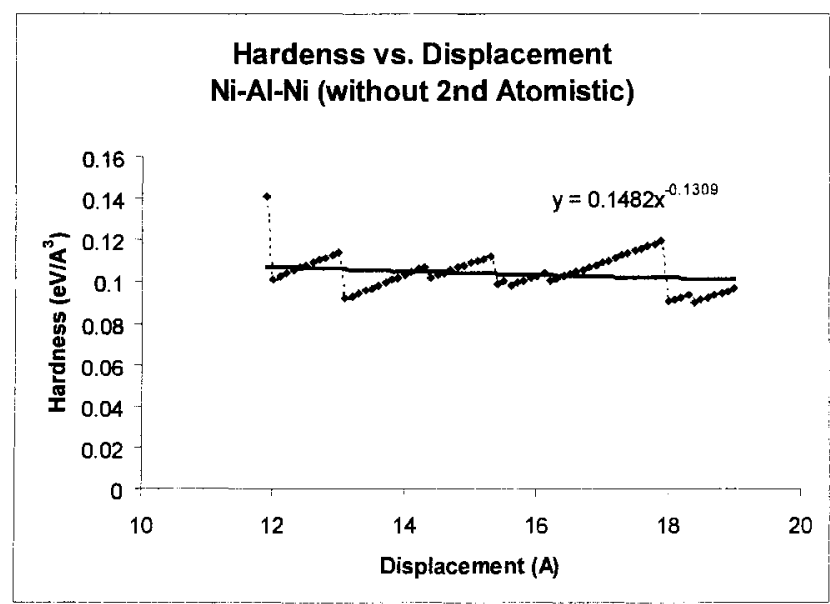

(c)

Figure 4.20: Hardness trends after first dislocation nucleation for different multilayer models. 


\section{Chapter 5}

\section{Conclusions and Future Recommendations}

\subsection{Summary of conclusions}

Nano-crystalline metals are gaining popularity among researchers and scientists, for both modeling and experiment, as they exhibit enhanced material properties which are highly desirable in many engineering applications. The purpose of this work was to investigate the underlying mechanisms and the influence of nanostructural defects in determining the nano-indentation response and dislocation plasticity associated with the nanolayered structures. Some interesting observations have been revealed upon completion of this work.

We basically examined two different types of bilayer models- a soft Al film on top of a hard $\mathrm{Ni}$ substrate (Al/Ni model) and a hard $\mathrm{Ni}$ film on top of a soft $\mathrm{Al}$ substrate ( $\mathrm{Ni} / \mathrm{Al}$ model). The behaviors of these two models were compared with the responses of single crystal pure aluminum and pure nickel. In both of these two types of bilayer models, the lattice constant mismatch between film and substrate led to the formation of misfit dislocations at the layer interface. These misfit dislocations initiated the plastic deformation and also played a key role in determining the subsequent overall deformation of the model. Moreover, the misfit dislocations acted as obstacles to the glide dislocation movement. During the indentation process, the 
movements of misfit dislocations were observed along the layer interface, either inward or outward of the center of the model, depending upon the placement of the material in the layers. Dissociation of the misfit dislocations, which can be viewed as a $2 \mathrm{D}$ analogue of cross-slip in 3D, occurred from the interface at certain stage of indentation, and in every bilayer model it happened into the aluminum layer (either film or substrate) due to its lower hardness compared to nickel. It had also been revealed that the stress field induced by the indenter dictated the movement of the misfit dislocations along the interface and their dissociations. When the indenter was brought in contact to the surface, it significantly altered the stress level of the atoms that were located near the surface and along the interface. The effect was found to be more intense especially at the locations where the misfit dislocations were located. However, the stress field of the indenter did not significantly affect the stress levels of the atoms that were located away from the surface or the interface. Load -displacement curves for each bilayer model showed good elastic response of the model at the initial stages of loading, whereas, the first drop in each curve was an indication of the initiation of plastic deformation of the models either by nucleation of a dislocation or by dissociation of a misfit dislocation from the interface. This phenomenon occurred at different level of load and different indentation depth for every case. Pure nickel was found to exhibit a higher load requirement than pure aluminum to nucleate the first dislocation. Similarly, the Ni/Al model exhibited a higher load requirement to initiate the dissociation of a misfit dislocation than the $\mathrm{Al} / \mathrm{Ni}$ bilayer model. The variation of hardness with indentation depth was measured by dividing the load by the projected contact area after every load step. It was found that the overall hardness of the bilayer models depend primarily upon the hardness of the film, although at higher penetration depths, the substrate effect cannot be ignored. Thus, by placing a hard film coating on a soft substrate enhanced the hardness and further enhancement was achieved by increasing the film 
thickness. The hardness trends after the first dislocation nucleation were dictated by simultaneous contributions from the indentation size effect due to the strain gradients and the hardening effect due to the high dislocation density. The hard substrate exhibited substantial resistance to dislocation movement and resulted in hardening when penetration depth was more than 50 percent of the film thickness. On the other hand, the soft substrate failed to resist dislocation movement and did not show any hardening effect even at a large penetration depth as the substrate also yielded along with the film. The responses of the pure materials were believed to be free from any effects of the substrate since the maximum indentation depths were very small compared to the overall film thickness. Easy glide of dislocations and dissociation events within pure aluminum resulted in lower dislocation density and indentation size effect at higher indentation depth. Whereas, the indentation size effect was almost mitigated by the effect of hardening due to the lack of dislocation movements and scarce dissociation events within single crystal pure nickel.

The multilayer models were constructed by placing two layers of film on top of a substrate, for example, $\mathrm{Al}$ and $\mathrm{Ni}$ films on top of $\mathrm{Al}$ substrate (Al-Ni-Al model) and $\mathrm{Ni}$ and $\mathrm{Al}$ films on top of $\mathrm{Ni}$ substrate ( $\mathrm{Ni}-\mathrm{Al}-\mathrm{Ni}$ model). An additional atomistic region was introduced in each of these two models to consider the full atomistic details (i.e. misfit dislocation, staking faults etc.) at the second interface. The necessity of this atomistic region was validated by constructing a third model, in which the second atomistic region was replaced by a continuum region ( $\mathrm{Ni}-\mathrm{Al}-\mathrm{Ni}$ without 2nd atomistic model), to see the effectiveness of the continuum models to elucidate the phenomena of dislocation-interface interactions at the second interface. Load-displacement plots for all multilayer models also exhibited good elastic response of the models before initiation of any plastic deformation. The Ni-Al-Ni model and Ni-Al-Ni without second atomistic model had almost the same response upto the points of first dislocation nucleation, but the amount of load drops at those 
points were different in these two cases. The higher load drop in the first model was attributed to the blocking of dislocations coming down from the top layers at the second interface and simultaneous dissociation event of a misfit dislocation from this interface. While in the second model, continuum description at the second interface failed to capture the picture accurately as dislocations coming down from the top layers were able to pass the interface quite easily and also yielded the substrate. The hardness trends after the first dislocation nucleation also exhibited their dependence upon simultaneous contributions from the indentation size effect and the hardening effect due to the high dislocation density. In the Ni-Al-Ni model, a hardening effect due to dislocation blocking was clearly seen, which mitigated the indentation size effect at a large penetration depth. On the other hand, this hardening effect was absent when atomistic details at the interface were ignored. Rather, gradual softening of the model indicated easy glide of the dislocations through the films and the substrate.

\subsection{Recommendations for future work}

There exist a range of possible extensions of the present study to get further insight into the indentation response of the nanolayered structures.

- A major important direction for future work is to extend the CADD method to a 3D formulation. That will require implementation of a parallel computing framework, but that will facilitate the investigation of real crystal structures (fcc, bcc, hcp etc.). In the 3D CADD framework, handling of dislocation loops wholly contained either in the atomistic region or in the continuum region may not be a problem but the major challenge will be in handling single dislocation loops located partly in the atomistic region and partly in the continuum region.

- The effect of different crystal orientations in different layers on the indenta- 
tion response should also be studied. In this present study, the same crystal orientation (100) was considered for each layer. Thus, the dislocations did not experience any resistance due to the slip plane mismatch while crossing the interface. But, it is a well known fact that materials possess different properties in different directions and their behaviors also vary tremendously with the change of the crystal orientation. Thus, modeling different crystal orientations for different layers will facilitate to reveal the effects of slip plane mismatches on dislocation movement across the interface in addition to the lattice constant mismatch and elastic constant mismatch. It is strongly believed that by selecting the crystal orientation properly in each layer, it is possible to create structures having properties even better than that of any of the constituent materials.

- It is also necessary to investigate the behavior of other metallic systems involving copper, silver, gold etc. Appropriate interatomic potentials for ternary systems may help to study models consisting of three different materials. Currently, work is being progressing using the EAM interatomic potential for aluminum, silver and copper developed by C.L. Rohrer [71]. This potential will allow us to create bilayer structures involving $\mathrm{Al}-\mathrm{Cu}, \mathrm{Al}-\mathrm{Ag}$ and $\mathrm{Ag}-\mathrm{Cu}$ and multilayered structures involving alternating layers of $\mathrm{Al}, \mathrm{Ag}$ and $\mathrm{Cu}$. Many interesting observations may come out upon revealing the properties associated with these materials. However, metal-nitride systems or nitride-nitride systems will require extensive modification of the existing CADD code and selection of appropriate interatomic potentials. This is because covalently bonded nitrides require higher order potentials.

- Dislocation nucleation and movement in the atomic and continuum regions during the unloading cycle can be analyzed to complete the indentation experi- 
ment. Previous study of nano-indentation on single crystal aluminum revealed some interesting observations including dislocation nucleation, annihilation, pile-up of materials and a 'Bauschinger' effect due to the reverse motion of the pre-existing dislocations upon removal of the load. In addition to these, the role of misfit dislocations and the interfaces in this reverse plasticity can be revealed upon completion of the unloading modeling of the layered structures. Moreover, to determine the stiffness and modulus of the layered structures it is necessary to analyze the unloading part of the load-displacement curves.

- To get a more realistic picture and to compare the results with experimental observations, it is necessary to increase the number of layers of the models. Typical multilayer coating structures are not limited to one or two layers of film on a substrate; sometimes they may consist of up to hundreds of layers. Moreover, the layer thickness should also be reduced, since most of the multilayer structures exhibit substantial strength and hardness enhancement at a bilayer period between 10 20 A. Defining separate atomistic region for each interface will definitely increase the complexity of the modeling. Instead, a single atomistic region can be constructed that will include all the interfaces. But handling this gigantic atomistic region in a single processor will require several days or even months computational time to solve one single problem. A parallel version of the CADD method is thus necessary to save computational time and to handle large number of atoms and nodes.

These proposed studies will help to reveal the potential to create a new class of high strength materials and will be a contribution towards further understanding of important aspects of materials science through computer simulation of deformation mechanisms in crystalline solids. 


\section{Bibliography}

[1] D.E. Kramer, M.F. Savage, A. Lin, and T. Foecke. Novel method for TEM charcterization of deformation under nanoindents in nanolayered materials. Scripta Materialia, 50(6):745-749, 2004.

[2] S.A. Barnett and A. Madan. Hardness and stability of metal-nitride nanoscale multilayers. Scripta Materialia, 50(6):739-744, 2004.

[3] I.A. Ovid'ko. Deformation of nanostructures. Science, 295(5564):2386, 2002.

[4] P.C. Yashar and W.D. Sproul. Nanometer scale multilayered hard coatings. Vacuum, 55:179-190, 1999.

[5] Q. Yang, C. He, L.R. Zhao, and J-P. Immarigeon. Preferred orientation and hardness enhancement of $\mathrm{TiN} / \mathrm{CrN}$ superlattice coatings deposited by reactive magnetron sputtering. Scripta Materialia, 46:293-297, 2002.

[6] Z. Shan, E.A. Stach, J.M. Wiezorek, J.A. Knapp, D.M. Follstaedt, and S.X. Mao. Grain boundary-mediated plasticity in nano crystalline nickel. Science, $305(5684): 654-657,2004$.

[7] K.W. Jacobsen and J. Sciotz. Computational material science: Nanoscale plasticity. Nature Materials, pages 15-16, 2002.

[8] D. Saraev and R.E. Miller. Enhanced hardness of copper crystals by nanometersized nickel coatings. submitted to Acta Mat., 2005. 
[9] S.I. Rao and P.M. Hazzledine. Atomistic simulation of dislocation-interface interactions in the Cu-Ni multilayer system. Phil. Mag. A, 80(9):2011-2040, 2000.

[10] Y.M. Wang, M.W. Chen, F.H. Zhou, and E. Ma. High tensile ductility in a nanostructured metal. Nature, 419:912-915, 2002.

[11] R.Z. Valiev, I.V. Alexandrov, Y.T. Zhu, and T.C. Lowe. Paradox of strength and ductility in metals processed by severe plastic deformation. J. Mater. Res., $17(1), 2002$.

[12] A.F. Voter. Inter atomic potentials for atomistic simulation. MRS bulletin, pages $17-19,1996$.

[13] A. Nakano, M.E. Bachlechner, T.J. Campbell, R.K. Kalia, A. Omeltchenko, K. Tsuruta, and P. Vashishta. Atomistic simulation of nanostructured materials. IEEE computational science and engineering, 5(4):68-78, 1998.

[14] R.E. Miller, W.A. Curtin, and L.E. Shilkrot. A coupled atomistics and discrete dislocation plasticity simulation nano indentation into single crystal thin films. Acta Mat., 52(2):271-284, 2004.

[15] L.E. Shilkrot, R.E. Miller, and W.A. Curtin. Coupled atomistic and discrete dislocation plasticity. Phys. Rev. Lett., 89(2):025501/1-025501/4, 2002.

[16] L.E. Shilkrot, R.E. Miller, and W.A. Curtin. Multiscale plasticity modeling: Coupled atomistic and discrete dislocation mechanics. submitted to J. Mech. Phys. Sol., 2003.

[17] Milton Ohring. The Materials Scinece of Thin Films. Academic Press, INC, San Diego, CA, 1992. 
[18] R.C. Cammarata and T.E. Schlesinger. Nanoindentation study of the mechanical properties of copper-nickel multilayered thin films. Appl. Phys. Lett., 56(19):1862-1863, 1990.

[19] Xi Chu and Scott A. Barnett. Model of superlattice yield stress and hardness enhancement. J. Appl. Phys., 77(9):4403-4411, 1995.

[20] P.B. Mirkarimi, L. Hultman, and S.A. Barnett. Enhanced hardness in lattice-matched single-crystal $\mathrm{TiN} / \mathrm{V}_{0.6} \mathrm{Nb}_{0.4} \mathrm{~N}$ superlattice. Appl. Phys. Lett., $57(25): 2654-2656,1990$.

[21] Thin Films. American Society for Metals, Ohio, 1964.

[22] R.A. Coombe, editor. The Electrical Properties and Applications of Thin Films. Sir Issac Pitman and Sons Ltd., London, 1967.

[23] L.I. Maissel and M.H. Francombe. An Introduction to thin films. Gordon and Breach, New York, N.Y., 1973.

[24] Anthony C. Fischer-Cripps. Nanoindentation. Springer, second edition, 2004.

[25] G.M. Pharr, W.C. Oliver, and F.R. Brotzen. On the generality of the relationship among contact stiffness, contact area, and elastic modulus during indentation. J. Mater. Res., 7(3):613-617, 1992.

[26] W.C. Oliver and G.M. Pharr. An improved technique for determining hardness and elastic modulus using load and displacement sensing indentation experiment. J. Mater. Res., 7(6):1564-1580, 1992.

[27] J. B. Pethica, R. Hutchings, and W. C. Oliver. Hardness measurements at penetration depths as small as $20 \mathrm{~nm}$. Phil. Mag. A, 48(4):593-606, 1983.

[28] M.F. Doerner and W.D. Nix. A method for interpreting the data from depthsensing indentation instruments. J. Mater. Res., 1(4):601-609, 1986. 
[29] S.V. Hainsworth, H.W. Chandler, and T.F. page. Analysis of nanoindentation load-displacement loading curves. J. Mater. Res., 11(8):1987-1995, 1996.

[30] N.A. Stelmashenko, M.G. Walls, M.L.Brown, and Y.V. Milman. Microindentations on W and Mo oriented single crystals: An STM study. Acta Melall. Mater., 41(1):2855-2865, 1993.

[31] M.S. De Guzman, G. Neubauer, P. Flinn, and W.D. Nix. The role of indentation depth on the measured hardness of materials. Mat. Res. Soc. Symp. Proc., 308:613-618, 1993.

[32] N. A. Fleck, G. M. Muller, M. F. Ashby, and J. W. Hutchinson. Strain gradient plasticity: Theory and experiment. Acta Met. et Mat., 42:475-487, 1994.

[33] N. A. Fleck and J. W. Hutchinson. Strain gradient plasticity. Adv. in Appl. Mech., 33:295-361, 1996.

[34] W.D. Nix and H. Gao. Indentaion size effects in crystalline materials: A law for strain gradient plasticity. J. Mech. Phys. Sol., 46(3):411-425, 1998.

[35] W.W. Gerberich, N.I. Tymiak, J.C. Grunlan, M.F. Horstemeyer, and M.I. Baskes. Interpretation of indentation size effects. J. Appl. Mech., 69:433-442, 2002.

[36] Chung-Jen Lu and D.B. Bogy. The effect of tip radius on nano-indentation hardness tests. Int. J. Sol. and Struc., 32(12):1759-1770, 1995.

[37] Yo-Han Yoo, Woong Lee, and Hyunho Shin. Effect of work hardening on the critical indentation limit in spherical nano-indentation of thin film/substrate systems. Surface and coatings technology, 179:324-332, 2004.

[38] J.A. Zimmerman, C.L. Kelchne, P.A. Klein, J.C. Hamilton, and S.M. Foiles. Surface step effects on nanoindentation. Phys. Rev. Lett., 87(16), 2001. 
[39] J.D. Embury and J.P. Hirth. On dislocation storage and the mechanical response of fine scale microstructures. Acta. Metall. Mater., 42:2051, 1994.

[40] A. Misra, J.P. Hirth, and H. Kung. Single-dislocation-based strengthening mechanisms in nanoscale multilayers. Phil. Mag. A, 82(16):2935-2951, 2002.

[41] T.G. Nieh and J. Wadsworth. Hall-petch relation in nanocrystalline solids. Scripta Metallurgica, 25:955-958, 1991.

[42] D. Hull and D.J. Bacon. Introduction to Dislocation. Springer, third edition, 1984.

[43] R.W. Siegel and G.E. Fougere. Nanophase Materials: Synthesis-PropertiesApplication. NATO-ASI Ser. E, Vol 260, Kluwer, Dordrecht, 1994.

[44] J. Lian, B. Baudelet, and A.A. Nazarov. Model for the prediction of the mechanical behaviour of nanocrystalline materials. Mater. Sci. Eng. A, 172:23-29, 1993.

[45] T. G. Langdon. The significance of grain boundaries in the flow of polycrystalline materials. Mater. Sci. Forum, 189-190:31-42, 1995.

[46] A.H. Chokshi, A. Rosen, J. Karch, and H. Gleiter. On the validity of the hallpetch relationship in nanocrystalline materials. Scripta Materialia, 23:1679$1684,1989$.

[47] J. Schiotz, Francesco D. Di Tolla, and K.W. Jacobsen. Softening of nanocrystalline metals at very small grain sizes. Nature, 391:561-563, 1998.

[48] J.S. Koehler. Attempt to design a strong solid. Phys. Rev. B, 2(2):547--551, 1970 .

[49] E.S. Pacheco and T. Mura. Interaction between a screw dislocation and a bimetallic interface. J. Mech. Phys. Sol., 17:163-170, 1969. 
[50] J.G. Sevillano. Strength of metals and alloys. Perganom, Oxford, 1980.

[51] U. Helmersson, S. Todorova, S.A. Barnett, and J.E. Sundgren. Growth of singlecrystal TiN/VN strained-layer superlattices with extremely high mechanical hardness. J. Appl. Phys., 62(2):481-484, 1987.

[52] T.D. de la Rubia and V.V. Bulatov. Materials research by means of multiscale computer simulation. MRS bulletin, pages 169-170, 2001.

[53] Dieter W. Heermann. Computer simulation method. Springer-Verlag, Germany, 1986.

[54] J. Hafner. Atomic-scale computational materials science. Acta Mat., 48:71-92, 2000 .

[55] D. Srivastava and S.N. Atluri. Computational nanotechnology: A current perspective. CMES, 3(5):531-538, 2002.

[56] Ronald E. Miller. On the generalization of continuum models to include atomistic features. PhD thesis, Brown University, 1998.

[57] M. S. Daw and M. I. Baskes. Embedded-atom method: Derivation and application to impurities, surfaces, and other defects in metals. Phys. Rev. B, 29:6443-6453, 1984.

[58] MA Xinling and Yang Wei. MD simulation for nanocrystals. Acta Mechanica Sinca, 19(6):485-507, 2003.

[59] A. Needleman and E. Van der Giessen. Discrete dislocation plasticity. Key Engineering Materials, 233-236:13-24, 2003.

[60] E. Van der Giessen and A. Needleman. Discrete dislocation plasticity: a simple planar model. Model. Simul. Mater. Sci. Eng., 3:689-735, 1995. 
[61] L.P. Kubin and G. Canova. The modelling of dislocation patterns. Scripta Metallurgica, 27:957-962, 1992.

[62] Hussein M. Zbib, Moono Rhee, and John P. Hirth. On plastic deformation and the dynamics of 3D dislocations. Int. J. Mech. Sci., 40(2-3):113-127, 1998.

[63] R.E. Miller and E.B. Tadmor. The quasicontinuum method: Overview, applications and current directions. J. Comp. Aid. Mat. Des., 9:203-239, 2002.

[64] E. B. Tadmor, M. Ortiz, and R. Phillips. Quasicontinuum analysis of defects in solids. Phil. Mag. A, 73(6):1529-1563, 1996.

[65] W.A. Curtin and Ronald E. Miller. Atomistic/continuum coupling in computational material science. Mod. Simul. Mater. Sci. Eng., 11:R33-R68, 2003.

[66] S. Kohlhoff, P. Gumbsch, and H. F. Fischmeister. Crack propagation in bcc crystals studied with a combined finite-element and atomistic model. Phil. Mag. A, 64(4):851-878, 1991.

[67] J.Q. Broughton, F.F. Abraham, N. Bernstein, and E. Kaxiras. Concurrent coupling of length scales: methodology and application. Phys. Rev. B, 60:23912403.

[68] Ronald E. Miller. Direct coupling of atomistic and continuum mechanics in computational material science. International Journal for Multiscale Computational Engineering, 1(1):57-72, 2003.

[69] A.F. Voter and S.P. Chen. Accurate interatomic potentials for $\mathrm{Ni}, \mathrm{Al}$ and $\mathrm{Ni}_{3} \mathrm{Al}$. Mat. Res. Soc. Symp. Proc., 82:175-180, 1987.

[70] M. S. Daw and S. M. Foiles. Dynamo version 8.7. FORTRAN code, 1994.

[71] C. L. Rohrer. Interatomic potentials for Al-Cu-Ag solid-solutions. Modeling Simul. Mater. Sci. Eng., 2:119-134, 1994. 